\title{
How to extend the chart of nuclides?
}

\author{
G. G. Adamian ${ }^{1}$, N. V. Antonenko ${ }^{1,2}$, A. Diaz-Torres ${ }^{3}$, S. Heinz ${ }^{4,5, a}$ \\ ${ }^{1}$ Joint Institute for Nuclear Research, 141980 Dubna, Russia \\ 2 Tomsk Polytechnic University, 634050 Tomsk, Russia \\ ${ }^{3}$ University of Surrey, Guildford, Surrey GU2 7XH, UK \\ ${ }^{4}$ GSI Helmholtzzentrum für Schwerionenforschung GmbH, 64291 Darmstadt, Germany \\ 5 Justus-Liebig-Universität Gießen, II. Physikalisches Institut, 35392 Gießen, Germany
}

Received: 20 August 2019 / Accepted: 22 December 2019 / Published online: 7 February 2020

(C) The Author(s) 2020

Communicated by N. Alamanos

\begin{abstract}
In the past 85 years the number of known nuclides increased by more than a factor of ten, resulting in 4000 presently known isotopes of 118 elements. This considerable progress we owe to the discovery of new reaction types along with the development of powerful accelerators and experimental techniques for separation and identification of reaction products. Model predictions indicate that still about 4000 further nuclides are waiting for their discovery. The vastest unexplored territory is located on the neutron-rich side in the upper half of the chart of nuclides and hides the answers to some of the most fundamental questions of nuclear physics like the limits of nuclear stability, element synthesis in the universe or stellar evolution. The access to these nuclei is presently limited by available beam intensities and/or the lack of appropriate methods for their production and identification. The latter concerns particularly new neutron-rich isotopes of transuranium and superheavy elements. To extend this area, the hope is presently based on multinucleon transfer reactions and on the application of fusion reactions with radioactive ion beams. But how promising are these approaches? Based on a survey of present-day knowledge, we will treat the questions where we currently are on our journey towards new territory on the chart of nuclides, how the chances are to gain new territory in the future and which challenges we will have to face.
\end{abstract}

\section{Contents}

1 Introduction . . . . . . . . . . . . . 2

2 Deep-inelastic collisions: complete fusion and transfer 3

2.1 Features and model concept . . . . . . . . 3

2.2 Experimental evidence for the model concept . 5 2.2.1 Observation of long-living rotating nuclear molecules ............. 5

a e-mail: s.heinz@gsi.de
2.2.2 DNS evolution pathways . . . . . . . 6

3 Theoretical approaches to deep-inelastic collisions . 7

3.1 Microscopical description of MNT . . . . . . 10

3.2 The nucleus-nucleus potential . . . . . . . 12

3.3 Transport coefficients .......... . 13

3.4 Excitation energy distributions, centroids of charge and mass distributions . . . . . . . . . . 14

3.5 Master equations for nucleon transfer . . . . 15

$3.6 Q_{g g}$ systematics . . . . . . . . . . . 17

3.7 Simplified statistical method . . . . . . . 18

4 Nucleosynthesis in transfer reactions . . . . . . . . 21

4.1 Early discoveries of new isotopes in MNT reactions ................ 21

4.2 Search for superheavy nuclei in MNT reactions 22 4.2.1 Early experiments . . . . . . . . . . 22

4.2.2 New model predictions . . . . . . . . 23

4.2.3 New experimental approaches and results 24

4.3 The region of neutron-rich $N=126$ nuclei . . 27 4.3.1 Model predictions . . . . . . . . . 27

4.3.2 Experimental state of the art . . . . . . 28

4.4 Shell effects in MNT reactions . . . . . . . . 30

4.5 Transfer-type reactions at intermediate energies 32

4.6 Incomplete fusion of light weakly bound nuclei 34

5 Nucleosynthesis with radioactive ion beams . . . . 36

5.1 Radioactive ion beams: production and yields . 36

5.2 How to get RIBs to Coulomb barrier energies? . 37

5.2.1 Post-acceleration of ISOL beams . . . . . 37

5.2.2 Deceleration of In-flight beams . . . . . . 38

5.3 Superheavy nuclei from fusion reactions with RIBs? ................ . . . 39

5.3.1 Expected cross-sections and yields . . . . 39

5.3.2 An "emergency solution"? . . . . . . . . 41

5.4 Existing and upcoming RIB facilities . . . . . . 41

6 The quest for new detection techniques . . . . . . . 42

6.1 High-precision mass measurements . . . . . 43 
6.2 Laser resonance ionization . . . . . . . . . . 43

6.3 Combination of mass separation and decay tagging .................. 43

7 Summary considerations . . . . . . . . . . . . 43

8 Ten supplementary questions . . . . . . . . . . . . . . 44

References . . . . . . . . . . . . . . . . . 46

\section{Introduction}

Figure 1 illustrates how the number of known nuclides increased in the past 85 years. In 1935, Giorgio Fea published the first compilation of then known nuclides, arranged in a two-dimensional map according to their proton and neutron numbers [1]. It comprised 327 isotopes of elements from hydrogen to uranium, most of them stable or close to the stability line. The base for the enormous increase of new isotopes in the following 85 years was the discovery of new reaction types. But their effective application for isotope production was only enabled by the development of powerful accelerators for heavy ions and sensitive separation and detection techniques for the reaction products (a review on the discovery of nuclides, related reactions and techniques can be found in $[2,3])$.

It was Ernest Rutherford who performed in 1919 the first man-made nuclear reaction in his lab in Manchester. By bombarding nitrogen with $\alpha$ particles from a radioactive source,

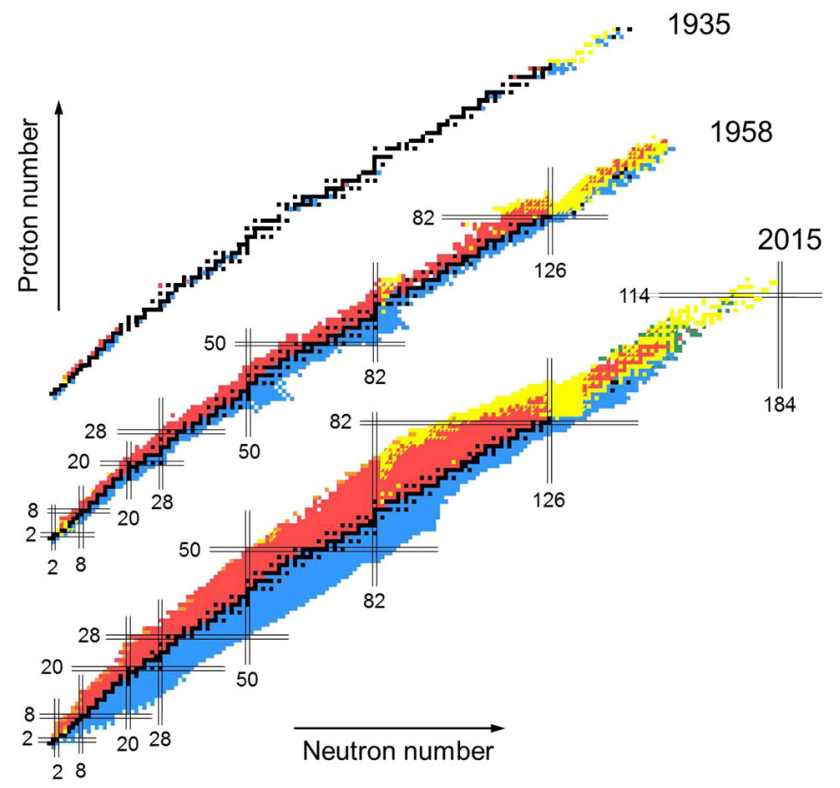

Fig. 1 The nuclide charts from 1935, 1958 and 2015 reflect the steep increase of known nuclides in the past 85 years. In 1935, 327 isotopes of 92 elements were recorded. The first Karlsruhe Chart of Nuclides, which appeared in 1958, comprised already about 1500 nuclides of 102 elements. Noteworthy are the two bulges on the neutron-rich side; the respective nuclei were produced as fission fragments. In the following 60 years, the number of known nuclides was more than doubled and the chart of 2015 contains 4000 different isotopes of 118 elements he triggered the fusion reaction ${ }^{4} \mathrm{He}+{ }^{14} \mathrm{~N} \rightarrow{ }^{18} \mathrm{~F}^{*} \rightarrow$ ${ }^{1} \mathrm{H}+{ }^{17} \mathrm{O}$. After the mid-1920s, the first ion accelerators appeared which enabled nucleosynthesis in fusion reactions with protons and light ions. The discovery of nuclear fission in the late 1930s $[4,5]$ entailed the observation of numerous neutron-rich fission fragments with mass numbers in a wide region around $A=100$. The mechanism of spallation/fragmentation was discovered in the mid-1940s [6] and later turned out to be very efficient for the production of isotopes all-over the chart of nuclides up to uranium. Around the same time, the upcoming powerful new accelerator facilities enabled fusion reactions with heavy ions. Their application allowed one to enter the region of transuranium nuclei up to the heaviest known elements. Till today, fusion, fission and fragmentation contribute most effectively to the production of new isotopes. The chart in Fig. 2 gives an overview which nuclides can be populated with these reactions. But it reveals also the vast, still empty area between the presently known isotopes and the expected driplines. The largest unexplored territory is located in the upper half of the chart, on the neutron-rich side. The access to these nuclei is presently limited by available beam intensities for fragmentation and fission reactions and by the bending of the stability line toward the neutron axis for fusion reactions. The increase of beam intensities is a main goal in accelerator laboratories worldwide. But to reach new neutron-rich transuranium nuclei, also new ways for their production are required. Two main approaches are presently discussed: the application of multinucleon transfer (MNT) reactions and the application of fusion reactions with radioactive ion beams (RIBs).

The discovery of MNT reactions dates back to the late 1960s when the upcoming accelerators for heavy ions

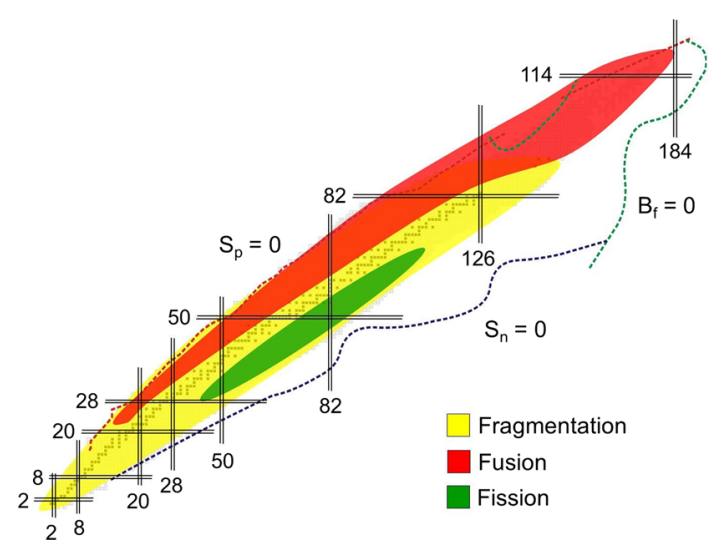

Fig. 2 Fragmentation, fusion and fission are presently the most effective reactions to produce (new) isotopes in the lab. In fragmentation reactions, neutron-rich as well as neutron-deficient nuclei up to uranium can be produced (yellow area). Fission leads to relatively neutron-rich intermediate heavy nuclei (green area). Fusion is presently the only method to synthesize transuranium and superheavy nuclei, but results in rather neutron-deficient reaction products due to the bending of the stability line (red area) 
allowed reactions to occur between complex projectile and target nuclei. Until then, the world of fusion reactions was simple. In collisions with very light projectiles, the orbital angular momentum $J$ determines if fusion can take place or not. For angular momenta up to a critical value, $J \leq J_{\text {crit }}$, the projectile is absorbed by the target nucleus and a compound nucleus $(\mathrm{CN})$ is formed. For $J>J_{\text {crit }}$ complete fusion is not possible anymore and only direct reactions or elastic scattering occurs. This picture was suddenly destroyed when scientists at the Joint Institute for Nuclear Research (JINR) in Dubna observed binary reaction products with broad mass, charge and angular distributions which emerged from deepinelastic low-energy heavy-ion collisions [7-13]. A bit later and independently, similar observations were also made in Orsay and Berkeley [14-18]. The observed characteristics suggested that deep-inelastic collisions (DIC) occupy an intermediate position between such different processes as direct reactions and $\mathrm{CN}$ reactions. There is no uniform terminology for this reaction type. Beside the denomination DIC, one can also find the expressions MNT, deep-inelastic transfer, quasi-fission, strongly damped collisions, relaxation phenomena and others. We will use the term MNT reactions.

From the very beginning it became obvious that MNT reactions are suitable to produce new isotopes; several neutron-rich nuclides of elements from carbon to thorium were discovered then. Also studies of MNT reactions with the goal to produce new transuranium and superheavy nuclei were performed in the 1970s up to the 1990s, but no new isotopes were observed in this region and finally, fusionevaporation reactions made the race at that time and the application of MNT reactions for isotope production abated for the moment. Ten years later the topic was raised again by the quest for suitable reaction types to access still unknown areas on the chart of nuclides. It started with new theoretical calculations and the particular interest to synthesize neutronrich medium-mass, heavy, and superheavy nuclei which are not reachable in fusion reactions with stable projectiles [1955]. Encouraged by cross-section calculations for mediummass and superheavy nuclei, MNT reactions were afterwards also suggested to synthesize neutron-rich nuclei along the $N=126$ shell, which are of great interest for understanding the astrophysical r-process path. These nuclei can principally be produced in fragmentation and fission reactions, but the calculated MNT cross-sections are promising large and it is worth to look closer if they can fulfill their promise for large yields. A completely different approach is the application of fusion reactions with RIBs, which is of particular interest for the synthesis of neutron-rich superheavy nuclei. The attention to this method increases with the upcoming powerful RIB facilities but its success depends crucially on available RIB intensities.

Meanwhile, MNT reactions and fusion reactions with RIBs are topical subjects in various laboratories around the world where appropriate separation and detection techniques are being explored $-\mathrm{a}$ challenging task regarding the expected tiny production cross-sections which can easily reach the sub-nanobarn scale. We should not forget that the reaction mechanism alone does not decide the success of the method. Equally important is the sensitivity of available experimental techniques for separation and detection.

But why do we make so big efforts to find new nuclides? A very basic goal is the better understanding and description of the nuclear force. Heavy nuclei are complex manybody systems and the long-range residual force of the strong interaction which binds the nucleons together can so far not be described in the framework of quantum chromodynamics. Therefore, many phenomenological models have been developed to treat nuclei in different regions of the chart of nuclides. Nuclei close to the stability line can be reasonably well described but for nuclei far from stability the lack of understanding increases. This makes obvious why nuclei close to the neutron and proton driplines or superheavy nuclei are highly desired to give new input for the models. New phenomena are expected for very exotic nuclei like for example neutron skins, appearance of new magic numbers and disappearance of the known ones, and new spherical shell closures in the region of superheavy nuclei. We should not forget that most of the astrophysical r-process path, which is responsible for the production of heavy elements in stellar nucleosynthesis, proceeds through terra incognita. According to a report by the National Research Council of the US National Academy of Sciences, the origin of heavy elements from iron to uranium remains one of the 11 greatest unanswered questions of modern physics [56]. So, there are many good reasons for making big efforts to find new nuclides.

\section{Deep-inelastic collisions: complete fusion and transfer}

\subsection{Features and model concept}

The large variances of mass (charge), energy and angular distributions of binary reaction products from DIC lead to the model concept of a molecule-like dinuclear system (DNS) which is formed by projectile and target nucleus during their interaction and enables the strong exchange of mass (charge) and energy between the reaction partners. This picture was postulated already soon after the discovery of DIC [57-65] and is still valid today. The schemes in Fig. 3 from early publications $[57,65]$ illustrate this process. The nuclear force leads to a sticking of projectile and target nucleus, forming the DNS. Sticking times are typically on the order $\left(10^{-21}\right.$ $10^{-20}$ ) s. Once the DNS is formed, the exchange of nucleons and energy between the reaction partners starts. In collisions with non-zero angular momentum, the DNS can also rotate about its center of gravity, where for long sticking times rota- 


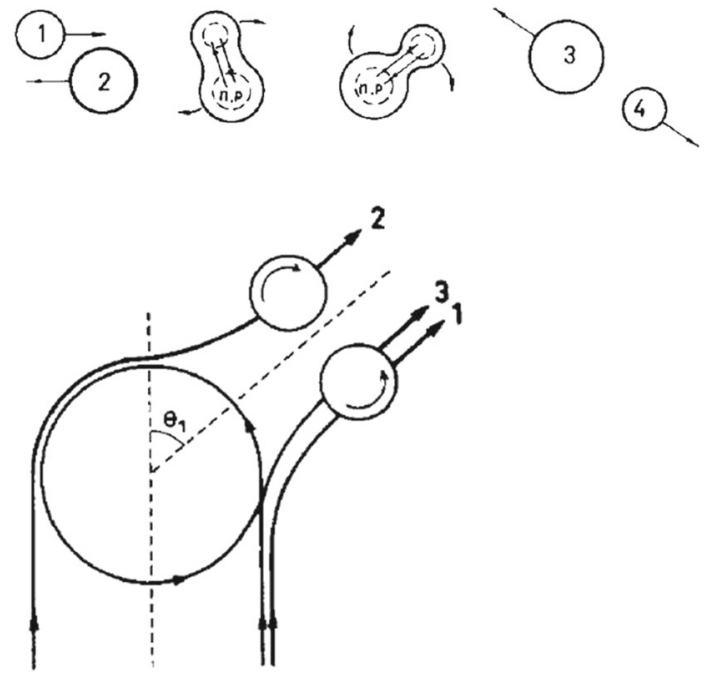

Fig. 3 Model concept of nuclear molecule formation and orbiting. The upper sketch is from an early publication of Volkov [57] and illustrates the idea of nuclear molecule formation as first step of deep-inelastic collisions at low energy. During the interaction, the molecule-like DNS evolves by continuous redistribution of nucleons, excitation energy and angular momentum between the nuclei before it finally decays. The lower drawing from Ref. [65] shows the concept of nuclear orbiting in DNS with angular momentum, resulting in three possible scattering trajectories. In all cases, the reaction products are emitted to the same angle, but the underlying reactions are quite different: 1 - quasielastic scattering in a grazing collision, 2 - deep-inelastic collision with sticking and rotation of the dinuclear system by $90^{\circ}, 3$ - deep-inelastic collision with full rotation of a long-living DNS

tions of $360^{\circ}$ or more can take place before the system decays. The DNS rotation explains the broad angular distributions of binary reaction products which resemble the angular distributions of fission fragments. The rotation of the DNS leads also to deviations of the reaction products trajectories with respect to the Coulomb trajectories of elastic scattering. DNS formation is usually accompanied by strong dissipation of kinetic energy which is mainly transformed into internal excitation of the system. This leads to the observed small kinetic energies of the reaction products with respect to the beam energy.

During formation and evolution of the DNS, there is continuous redistribution of nucleons, excitation energy and angular momentum between the two nuclei. The equilibrium between proton and neutron numbers occurs very fast in collisions of light nuclei and continuously proceeds in reactions with heavy nuclei [66-69]. As it evolves, the DNS can pass with some probability through any macroscopic configuration allowed by the conservation laws for particle number, charge and total energy. In the utmost case, the system can fuse and ends up in a compound nucleus. The two possible pathways of DNS evolution are illustrated in Fig. 4. If the system overcomes the fusion barrier, it reaches complete statistical equilibrium with respect to all degrees of

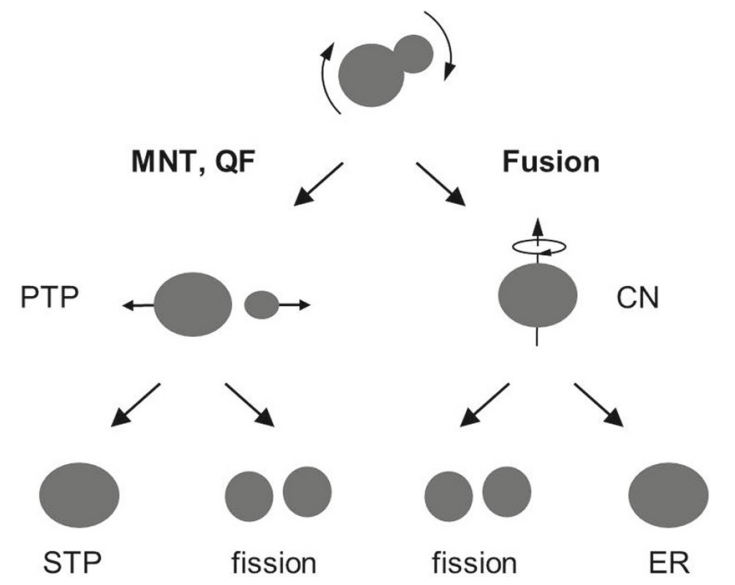

Fig. 4 The two possible evolution paths of nuclear molecules: once the molecule-like DNS is formed, it can undergo complete fusion, resulting in a $\mathrm{CN}$. The de-excitation of the $\mathrm{CN}$ proceeds either by evaporating nucleons and $\gamma$ rays, leaving an evaporation residue (ER) or by fission. Alternatively, the DNS can scission before reaching the $\mathrm{CN}$ state. Before scission, large amounts of nucleons can be exchanged between the reaction partners (multinucleon transfer MNT/quasi-fisson, QF). When the system breaks up, the still excited projectile-like and target-like primary transfer products (PTP) are emitted. Their de-excitation takes place via evaporation of nucleons and $\gamma$ rays leading to the secondary transfer products (STP) or, particularly in the case of heavy nuclei, by fission

freedom, forming a $\mathrm{CN}$. The $\mathrm{CN}$ can de-excite either by emitting nucleons, nucleon clusters and/or gamma rays or by fission ("fusion-fission"). Alternatively, the DNS can decay before it reaches the $\mathrm{CN}$ state. The decay occurs either for static reasons (dominance of Coulomb repulsion between the interacting nuclei) or for dynamical reasons (coupling of modes of motion). Before decay, large numbers of nucleons can be exchanged between the two nuclei and lead to reaction products far from the original projectile and target nuclei (MNT or quasi-fission reactions). This allows us to hope that also new exotic isotopes are among these products. After scission, the projectile-like and target-like MNT products ("primary transfer products") still carry the excitation energy of the DNS, which was distributed among them during the sticking time. Like in the case of a $\mathrm{CN}$, the de-excitation can take place by emission of nucleons and gamma rays leading to residual MNT products in their ground state ("secondary transfer products"). Alternatively, the excited primary MNT products can undergo fission for which the probability increases with increasing mass, spin, and excitation energy of the nuclei. According to this picture, complete-fusion and MNT reactions are closely related processes, which originate both from the formation of a dinuclear system. 


\subsection{Experimental evidence for the model concept}

\subsubsection{Observation of long-living rotating nuclear molecules}

A key process to validate the model concept of DIC is the experimental observation of long-living rotating DNS. In one of the earliest experiments, $\mathrm{Ar}+\mathrm{Th}$, sticking and orbiting of the nuclei was already indicated by the angular and energy distributions of binary reaction products [11]. Related to these observations, the possible existence of nuclear molecules was postulated then in Ref. [65]: "The idea of nuclear orbiting in collisions with angular momenta close to the critical value implies another interesting effect which eventually could be found experimentally. If some of the colliding systems survive more than half of a revolution, ... such a phenomenon would strongly indicate the existence of nuclear molecules rotating in the dynamical force equilibrium".

In the following decades, nuclear orbiting was observed in many further experiments and various collision systems, but signatures from DNS which performed "more than half of a revolution" were only found recently in the velocity spectra of target-like MNT products from collisions of $\mathrm{Ni}+\mathrm{Pb}(Z=110)$ at the GSI velocity filter SHIP [70] (Fig. 5). The spectra reveal a double peak structure consisting of a pronounced maximum at velocities larger than 1.5 times the $\mathrm{CN}$ velocity $v_{\mathrm{CN}}$ and a small peak at velocities $v<0.5 v_{\mathrm{CN}}$. For each observed nuclide the two peaks are located symmetrically with respect to the $\mathrm{CN}$ velocity. The peak positions and intensities shift systematically with the proton number of the MNT products. The most consistent explanation for the observed phenomenon is the formation of molecule-like rotating DNS which live sufficiently long to perform rotations by at least $180^{\circ}$. The high-velocity peak then originates from MNT products which were created in central collisions, without rotation. After scission of the DNS the projectile-like nucleus is emitted in backward direction and the target-like nucleus with velocity $v^{\prime}$ in beam direction, resulting in a peak at $v=v^{\prime}+v_{\mathrm{CN}}$ in the lab system. If the DNS has angular momentum, it will rotate about its center of gravity. After half of a revolution, the DNS is again aligned parallel to the beam axis where, however, projectile and target nucleus have changed their positions like indicated by the small drawings on top of Fig. 5. If reseparation of the DNS occurs in this orientation, the target-like nucleus is emitted in forward direction with a reduced velocity $v=-v^{\prime}+v_{\mathrm{CN}}$. Reaction products from other rotation angles are not accepted by the velocity filter which covers only forward angles of $(0 \pm 2)^{\circ}$.

But the velocity spectra provide still much more information about DNS evolution. Since the peak positions are directly related to the kinetic energy of the measured targetlike MNT products, they reveal the total kinetic energy (TKE) in the exit channel if one assumes a binary reaction process.

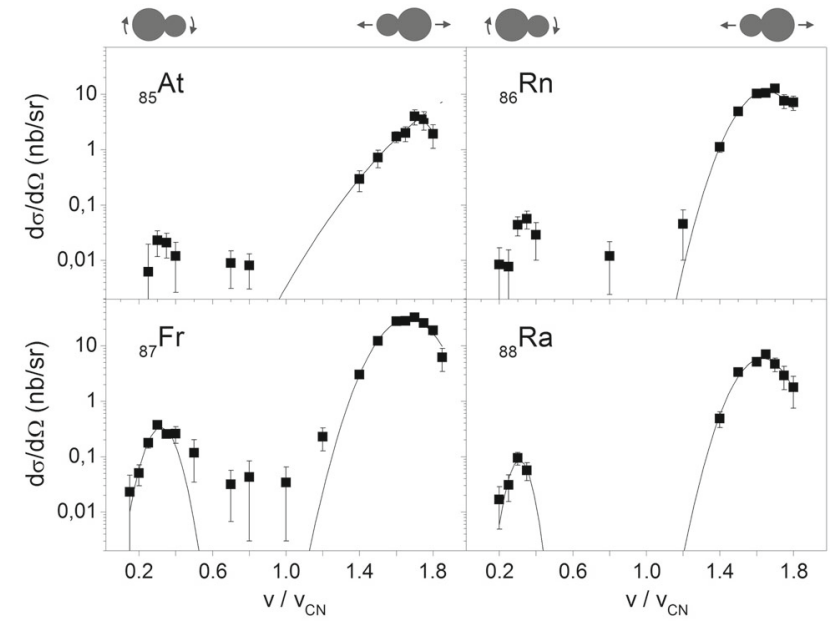

Fig. 5 Velocity spectra of At, Rn, Fr and Ra isotopes produced in multinucleon transfer reactions of ${ }^{64} \mathrm{Ni}+{ }^{207} \mathrm{~Pb}$ at $5.92 \mathrm{MeV} /$ nucleon [70]. The velocities are given in units of the compound nucleus velocity $v_{\mathrm{CN}}$. The peaks at small velocities, $v \approx 0.3 v_{\mathrm{CN}}$, are most likely the traces of long-living nuclear molecules which rotate by angles of $180^{\circ}$ or more

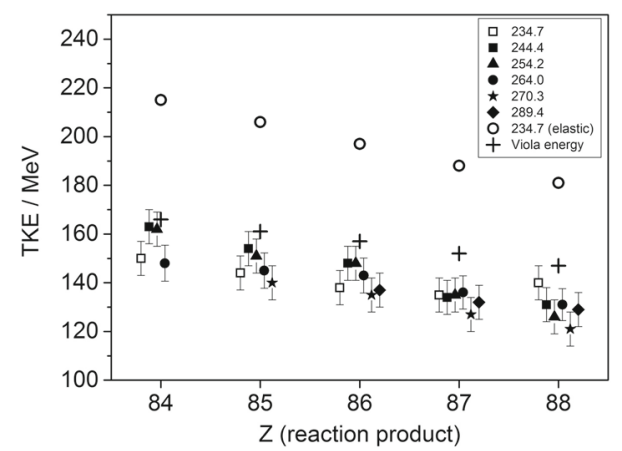

Fig. 6 Measured [71] total kinetic energy (TKE) as a function of the proton number $Z$ of multinucleon transfer products created in reactions of ${ }^{64} \mathrm{Ni}+{ }^{207} \mathrm{~Pb}$ at beam energies of $4.80,5.00,5.20,5.40,5.53$ and $5.92 \mathrm{MeV} /$ nucleon. The beam energies in the center-of-mass frame are given in the inset. The data points for a fixed $Z$ but for different beam energies are plotted with an offset for better discrimination. The energies expected for asymmetric fission fragments of the respective compound nucleus ${ }^{271}$ Ds according to the Viola systematics are represented by crosses. The open circles denote the TKE values expected from elastic kinematics at the lowest beam energy

The TKE values obtained in this way are shown in Fig. 6 as a function of the proton number $Z$ of the target-like MNT product for six different beam energies from 4.80 to 5.92 $\mathrm{MeV} /$ nucleon. In all reactions leading to nuclei with $Z \geq 84$, the TKE values are independent of the beam energy and they are located at or below the expected energy of fission fragments ("Viola energy") [72] from the respective compound nucleus ${ }^{271}$ Ds. This indicates that the DNS behaves already quite similar to a compound nuclear system. Namely, that full dissipation of kinetic energy took place and it "forgot" the entrance channel properties. In this case, the TKE val- 
ues, which decrease continuously with increasing number of transferred protons, are determined by the Coulomb barrier of the emitted MNT products at the scission point and reveal a strong deformation of the DNS before reseparation. From the internuclear distance which can be determined from TKE, quadrupole deformations of $\beta_{2} \approx 0.4$ are deduced [70]. Further, the intensity ratios of the two velocity peaks reveal the lifetimes (i.e. sticking times) of the DNS if one assumes that the DNS decay follows the decay law (details of the method are described in [70]). With the same "velocity filter method", weak signals from rotating DNS were also observed in the much heavier system $\mathrm{Xe}+\mathrm{Pb}(Z=136)$ [73]. This reveals that DNS formation takes still place in systems with proton numbers far beyond the region of known superheavy nuclei, where the fusion probability is already negligibly small. And even one of the heaviest systems, $U+U(Z=184)$, which was investigated in the late 1970s at GSI [74] and 30 years later at the GANIL VAMOS spectrometer [75], reveals signatures of noticeable time delays during the interaction, correlated with a large mass flow and strong energy dissipation. This behavior of the two heavier systems is particularly interesting if one considers them for synthesis of new exotic MNT products. We will come back to this later.

The direct comparison of these three systems [73] leads to astonishing results. Figure 7 shows the evolution of energy dissipation, interaction times and shapes during nuclear contact as a function of the net number of transferred nucleons $\mathrm{d} A$. The similarity of the qualitative behavior is obvious and reveals two phases of the reaction. In each system, the first phase is characterized by strong dissipation of kinetic energy (total kinetic energy loss, TKEL), which is revealed by the steep slope of the TKEL $/ E_{\text {c.m. }}$. curves in Fig. $7 \mathrm{a}$. In this phase only a small number of nucleons, not more than $5 \%$ of the total $A$, is exchanged between the two nuclei. Also interaction times and deformations increase strongly in that part of the curves (Fig. 7b, c) indicating that the process is time consuming. In the second phase, the deep-inelastic regime is entered and the situation reverses. Energy dissipation and interaction times become more and more independent of the number of transferred nucleons. Also the shapes change more moderately. It seems that large numbers of nucleons now flow with small friction and small time consumption. The system is obviously starting to evolve freely in the mass asymmetry coordinate. Amazing is that the transition from phase 1 to phase 2 seems to occur after the transfer of about $5 \%$ of the total nucleons, independent of the collision system.

\subsubsection{DNS evolution pathways}

Other important evidence for the model concept of DIC is the experimental verification of the different pathways of DNS evolution as sketched in Fig. 4. This can be done by studying quasi-fission $(\mathrm{QF})$ and fusion-fission (FF) reac-

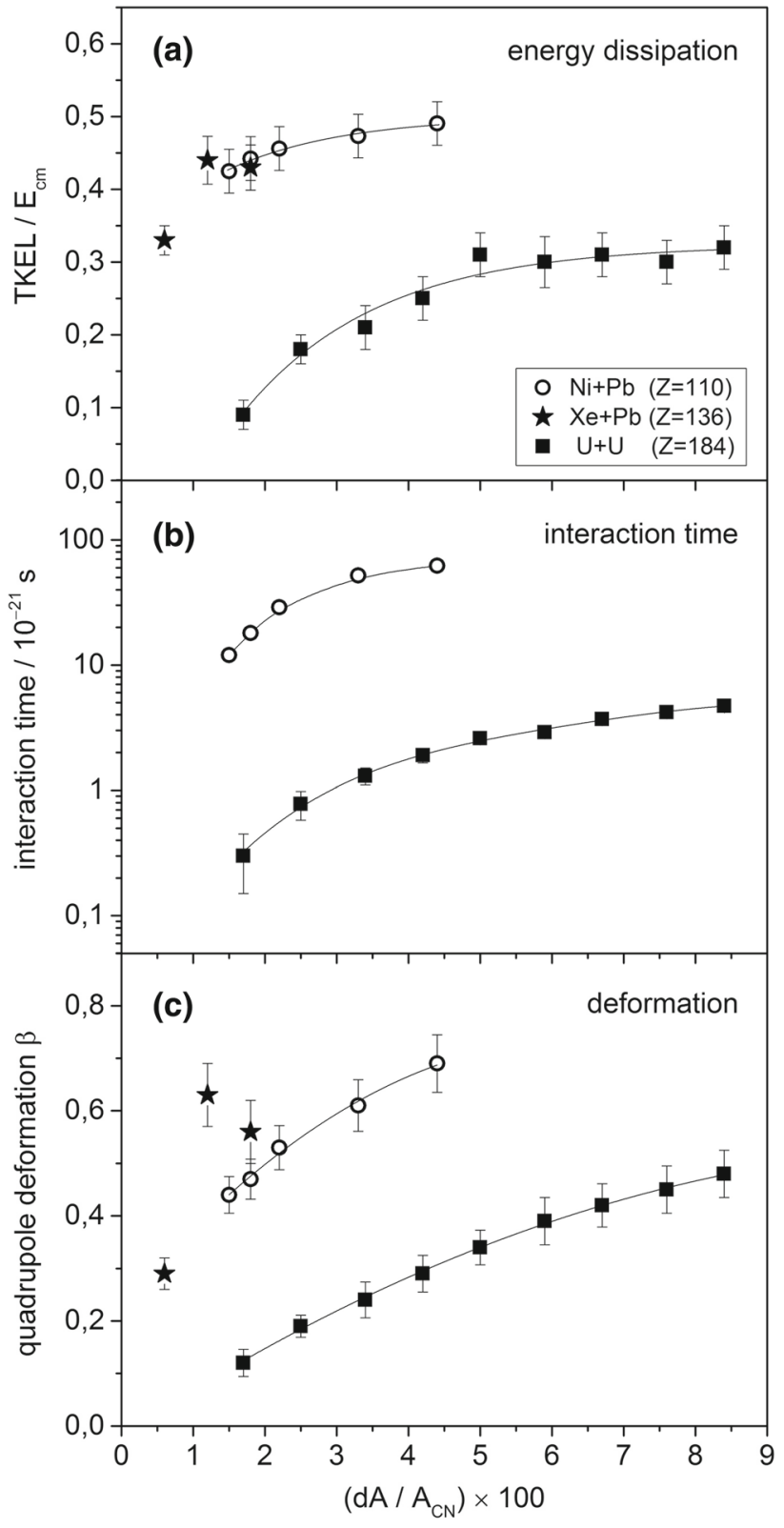

Fig. 7 Total kinetic energy loss (a), interaction times (b) and deformations of the exit channel nuclei at the scission point (c), for collisions of $\mathrm{Ni}+\mathrm{Pb}(Z=110), \mathrm{Xe}+\mathrm{Pb}(Z=136)$ and $\mathrm{U}+\mathrm{U}(Z=184)$ at energies of about $10 \%$ above the Coulomb barrier. The energy dissipation is given in terms of the total kinetic energy loss TKEL $=E_{\text {c.m. }}-$ TKE. For better comparison of the systems, TKEL is divided by the respective center-of-mass energy $E_{\mathrm{c} . \mathrm{m}}$. The $x$-axis represents the net number of transferred nucleons $d A$ divided by the total nucleon number $A_{\mathrm{CN}}$ of the respective compound system; $\mathrm{d} A / A_{\mathrm{CN}}$ is given in percent

tions, and there are indeed numerous experimental data [76103] which reveal these pathways. The tiny cross-sections of superheavy fusion products $[104,105]$ indicate already that the majority of DNS never ends up in a fusion-evaporation residue. Because the DNS formation cross-sections are large and reach several $100 \mathrm{mb}$ up to barns [76], it is obvious that 


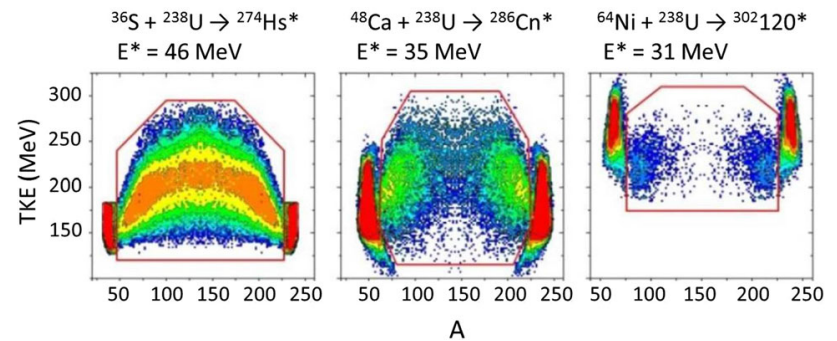

Fig. 8 Example spectra from Ref. [80] showing the mass $(A)$ versus TKE of binary reaction products from collisions of $\mathrm{S}+\mathrm{U}(Z=108)$, $\mathrm{Ca}+\mathrm{U}(Z=112)$ and $\mathrm{Ni}+\mathrm{U}(Z=120)$ measured with the CORSET setup of JINR. The respective excitation energies of the composite systems are indicated on top of the figures. The red frames mark fission-like events which comprise contributions from QF and FF

losses on the way to the fusion-evaporation residue must be huge. Presently, there are groups at JINR, at the Japan Atomic Energy Agency (JAEA) in Tokai, and at the Australian National University (ANU) in Canberra, who make extensive studies of QF and FF reactions. Their experimental setups are based on similar principles. The detection systems allow one to measure the time of flight (TOF) and energy $E$ of the reactions products, from which their mass $A$ and total kinetic energy TKE in the exit channel is obtained. In addition, the setups allow for the measurement of the angular distributions of the reaction products.

At JINR, QF and FF reactions in (super)heavy collision systems are studied for many years with the two-arm TOF$E$ detection system CORSET [77]. The about $0.5 \mathrm{~m}$ long detector arms can be positioned at different angles to measure also the angular distributions of the reaction products. The obtained mass resolution is $\Delta A / A \approx 1.5 \%$ which corresponds to (3-4) mass units for very heavy nuclei (also the JAEA and ANU setups allow for similar resolutions). Figure 8 shows example spectra of $A$-TKE distributions of binary reaction products measured with the CORSET setup in collisions of $\mathrm{S}+\mathrm{U}(Z=108), \mathrm{Ca}+\mathrm{U}(Z=112)$ and $\mathrm{Ni}+\mathrm{U}(Z=120)$ [80]. A pronounced feature of both, $\mathrm{QF}$ and FF is the strong dissipation of kinetic energy which accompanies these reactions leading to low TKE values with respect to the incident energy. Their most pronounced difference is in the $A$ distributions. The mass distributions of $\mathrm{QF}$ fragments are mainly asymmetric with maxima in the vicinity of the original projectile and target nuclei while FF leads to symmetric mass distributions which peak at $\left(A_{1}+A_{2}\right) / 2$. However, there is also an overlap of the $\mathrm{QF}$ and FF distributions in the sense that QF can contain symmetric components (from long-living DNS) and FF asymmetric ones, leading to uncertainties if one uses the measured distributions to quantify the individual contributions of QF and FF in a specific reaction.

By trend, a decrease of the FF component with increasing $A$ (and $Z$, respectively) is observed, like in Fig. 8. This

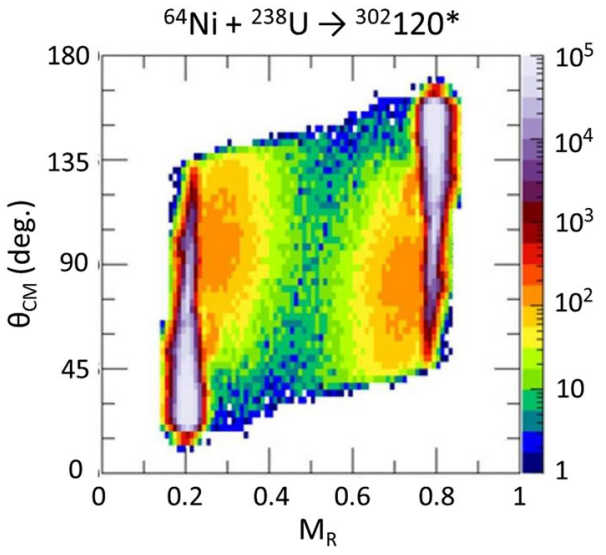

Fig. 9 Mass-angle distributions of binary reaction products from collisions of $\mathrm{U}+\mathrm{Ni}$ at a center-of-mass energy of $288 \mathrm{MeV}$ measured at the Australian National University ANU [83]. The masses are given as mass ratio $M_{\mathrm{R}}$ between the two reaction products at the scission point of the DNS. The rather narrow angular distribution and $M_{\mathrm{R}}$ close to the values of the entrance channel nuclei suggest short DNS lifetimes and $\mathrm{QF}$ as origin of the reaction products

is explainable with the increasing Coulomb repulsion which more and more prevents $\mathrm{CN}$ formation and drives the DNS to scission before full statistical equilibrium is reached. Further it is noticed that the FF branch increases with increasing beam energy. This can be explained with the increasing nuclear overlap which boosts $\mathrm{CN}$ formation. Collection of data for a variety of different collision systems and at different beam energies allows for the quantification of the individual contributions of QF and FF pathways (see e.g. [79,82]).

If one represents the $A$ distributions as a function of scattering angle, like it is usually done by the ANU group, one obtains in addition the information on DNS lifetimes. Figure 9 is an example for such mass-angle distributions (MAD) measured with the ANU setup for collisions of $\mathrm{Ni}+\mathrm{U}(Z=120)$ [83]. Principally, the width of the angular distributions increases with lifetime of the DNS. The MAD of the quite heavy $\mathrm{Ni}+\mathrm{U}$ system in Fig. 9 reveals that the angular distributions are relatively narrow and the masses of the reaction products mostly in the vicinity of the projectile and target masses. This indicates rather short DNS lifetimes and only small contributions from FF.

\section{Theoretical approaches to deep-inelastic collisions}

The dynamics of the DNS is described by two main degrees of freedom, namely, by (a) the relative motion of the fusing nuclei, and (b) the mass and charge transfer between the nuclei. Additional degrees of freedom are the deformations and orientations, the neck degree of freedom, internal excitations and others. The mass and charge transfer is usually described in coordinates of mass and charge asymmetry 
$\eta_{A}=\left(A_{1}-A_{2}\right) /\left(A_{1}+A_{2}\right)$,

$\eta_{Z}=\left(Z_{1}-Z_{2}\right) /\left(Z_{1}+Z_{2}\right)$,

where $A_{1}, A_{2}$ and $Z_{1}, Z_{2}$ are the mass and charge numbers of the two clusters. The coordinates $\eta_{A, Z}$ vary in the interval $(-1,1)$, where $\eta= \pm 1$ means that either $A_{1}=0$ or $A_{2}=0$, which is interpreted as complete fusion. In the case $\eta=0$ ( $A_{1}=A_{2}, Z_{1}=Z_{2}$ ), the DNS consists of two symmetric clusters. The potential energy determines the dynamics of the reaction. It can be calculated with the Strutinsky method where the potentials contain the liquid drop (LD) and shell effects, which are usually determined by the two-center shell model of Maruhn and Greiner [106].

One can distinguish the present theoretical approaches in phenomenological (microscopic and collective) and selfconsistent. Both describe MNT and fusion reactions fairly well, but the phenomenological models are presently more widely used. Their common ground is the formation of a DNS and its trapping in the minimum of the nucleus-nucleus potential as first step of the reaction. Resulting from this, the theoretical study of the DNS dynamics allows one to consider within a unique approach MNT and fusion reactions. Since the nuclei have internal structure, which is quite complicated at the considered excitation energies, some simplifications are required for choosing the most probable path of evolution in the configuration space, instead of considering all possible paths, and for introducing macroscopic characteristics (particle flux, friction, shape evolution, etc.). Dynamical and statistical characteristics are manifested to different degrees at different stages of the collision. This makes it possible to model separately each stage of the collision and also simplifies the theoretical treatment.

The basis for an analysis of the above described effects is the microscopic model. In the microscopic theory, the evolution of the DNS can be determined either by the timedependent Schrödinger equation for the wave function of the system or by the Liouville equation for the density matrix. One cannot solve these equations without making simplifying assumptions. Moreover, the exact solution of these equations would give more information than can be experimentally verified. The successes of relatively simple phenomenological and semi-microscopic models [107-115] indicate that not all degrees of freedom have a significant effect on the dynamics of the system. In these models, only certain collective (macroscopic) degrees of freedom are considered, which are chosen a priori, on basis of the requirements for the interpretation of experimental data. The number of collective degrees of freedom taken explicitly into account can be reduced by using experimentally established differences in their relaxation times.

The task of a microscopic theory is to find the effective Hamiltonian of the collective motion and its coupling with the internal degrees of freedom. This coupling is a source of irreversible processes in the DNS such as transfer of mass, charge, etc. The existing models differ in the internal excitations that they introduce explicitly or implicitly. They may be coherent and incoherent particle-hole excitations and transfers of nucleons or clusters of nucleons [116]. The first attempt was made in the model of dissipative diabatic dynamics $[117,118]$. This model is restricted solely by the form of the coherent motion of the nucleons, their diabatic motion in the changing two-center mean field. The process of excitation of the nuclei includes only particle-hole states whose diabatic levels cross during the motion of the nuclei. Because of the diabatic nature of the single-particle motion, the coupling between the collective and internal degrees of freedom is coherent and nonlinear, in contrast to the linear-response theory. In the dissipative limit, the friction in the dissipative diabatic dynamics model is equivalent to one-body friction ("wall" formula [119]).

There are two approaches, which start from opposite assumptions, to describing the establishment of equilibrium in the DNS. In the first approach, the exchange of nucleons is described as a stochastic process without inertial effects ("overdamped" regime) [66]. In the second approach, the charge asymmetry is regarded as a collective mode $[64,120,121]$. In the fragmentation theory [120,121], the evolution of the system along the mass asymmetry coordinate is determined by the time-dependent Schrödinger equation. Integrating this equation in the classical trajectory of relative motion of the colliding nuclei, one can determine the width of the mass (charge) distribution. However, the quantum fluctuations, which can be important, are ignored in this adiabatic treatment.

The transport (stochastic) models are widely used to describe DIC [122-129]. The exchange of mass and charge and the transfer of energy and angular momentum from the relative motion to internal degrees of freedom can be successfully interpreted as a diffusion process. By its nature, the interaction of nuclei is a non-equilibrium statistical process: the DNS is not only excited but also relaxes to complete statistical equilibrium. In comparison to the Schrödinger equation, the transport equations describe the irreversible evolution of a probability distribution and not an amplitude. All transport theories proceed from a division of the degrees of freedom of the system into a slow macroscopic degree and a rapidly relaxing internal degree. By using explicitly the statistical hypotheses for the interaction operator of the collective and the internal degrees of freedom, one can deduce the kinetic equations from the Liouville equation of motion and obtain the microscopic transport coefficients. In the macroscopic diffusion models, the transport coefficients are assumed to be proportional to the ratio of the phase spaces of the states of excited system. This means that the direction of nucleon transfer is determined by the balance between the total energies of different configurations of the DNS. However, the 
disregard of nuclear shell structure limits the description of experimental data. For example, the diffusion models which use only a potential-energy surface cannot explain the strong growth in the variance of the mass distributions along with a weak change of the mean masses of the reaction partners [130].

There are various transport models such as the randommatrix approximation $[122,127]$, the model of one-body dissipation $[128,129]$ and the linear-response model [126]. The main kinetic equation in these approaches is the master equation or Fokker-Planck equation for the distribution function of collective coordinates and conjugate momenta in the phase space of the collective degrees of freedom. In the microscopical models, the transport coefficients of the Fokker-Planck equation have been obtained. In these models, the internal system is described as a sum of independent internal subsystems of each of the nuclei. In Refs. [124,125], there are incoherent particle-hole excitations and exchange of nucleons between the nuclei due to non-diagonal matrix elements of the single-particle potential. The model of Ref. [128] considers only exchange of nucleons and is a microscopic analog of the classical picture of the exchange of particles through a window during the collision of nuclei [119]. The statistical hypothesis enters the model together with the assumption of rapid randomization of nucleon motions in each nucleus. The relative simplicity of the models and their success in describing the loss of kinetic energy ("window" formula) and the widths of the mass (charge) distributions of the reaction products are intriguing.

In the mentioned models, shell effects are not explicitly treated and the details of single-particle spectra of reaction partners are not considered. The matrix elements for nucleon transition are determined by averaging over all shell configurations. An influence of the structure of interacting nuclei is manifested only in a strong dependence of the transport coefficients on the density of the single-particle levels. In Ref. [125], the interacting nuclei are treated in the Fermi-gas approximation, and that means that the structure of nuclei is taken into account only in an averaged manner through the ground-state energy and the level density parameters. The approximation is not based on the perturbation theory, in contrast to the model of Ref. [128], in which the coupling between the collective and internal motions is described in the first order of perturbation theory ("weak-coupling" limit). The transport model of Ref. [128] includes (classically) dynamical effects, whereas the model $[124,125]$ does not consider explicitly the relative motion of the nuclei.

In the approach based on the linear-response theory [126], the main assumption is that in each point of the classical trajectory the internal system is close to thermodynamical equilibrium. In this case one can calculate the deviation of the density matrix of the internal system from equilibrium with the perturbation theory. The linear-response theory is formu- lated in the quasi-adiabatic approximation, i.e. the model is valid only for small collective velocities. The excitation of the internal system (incoherent particle-hole excitations in a mean-field potential) is generated after each infinitesimally short time interval by a change in the mean field of the DNS. The relative motion of the nuclei, the mass (charge) asymmetry, and the shapes of the nuclei are taken into account in this model. The macroscopic and microscopic degrees of freedom are coupled by a friction tensor. The dissipation in the linear-response theory is a quantum-mechanical version of the classical one-body friction ("wall" formula).

The assumption of the local equilibrium in the DNS is a good approximation for the final stage of the reaction but is not correct for the description of the initial stage. At the initial stage there are strong coherent excitations, which decay into complex incoherent states during the time in which the local equilibrium is established. To take into account these effects, the modified Fokker-Planck equation was obtained in [131].

In heavy-ion reactions at energies of a few $\mathrm{MeV} /$ nucleon above the Coulomb barrier, the DNS can be characterized as a system of non-interacting particles in a common timedependent mean field, since the average velocity (Fermi velocity) of the nucleons is much larger than the relative velocity of the ions. For the description of the initial stage of the reaction, the most general microscopic approach is through the solution of the multi-particle Schrödinger equation in the time-dependent Hartree-Fock (TDHF) approximation [132-134]. The TDHF contains a description of all degrees of freedom that are considered in the transport models. However, there are certain problems to separate the macroscopic observables, owing to the nonlinearity of the dynamical equations. With the TDHF one can obtain a good description of fission and DIC, the deflection functions, the dissipation of energy and relative angular momentum, the centroids of the mass (charge) distributions, etc. However, the calculated variances of the mass (charge) distributions are several times smaller than the experimental ones. The TDHF is also unable to describe well the fusion cross-section. Another serious shortcoming of the TDHF in the theory of nuclear reactions was its inability to take into account fluctuations of the mean field. In spite of the recent progress, the TDHF method is still time consuming for consideration of collisions of deformed nuclei. There are some alternative approaches based on the TDHF, such as the adiabatic time-dependent Hartree-Fock approximation (ATDHF) and semi-microscopic hydrodynamical models [135].

The multistage processes of heavy-ion collisions can be consistently described in the framework of the coupledchannels method [136]. For simplicity, one usually models the coupling of the channels by means of friction forces [129] and considers the collective coordinate coupled with the internal ones in the quantum diffusion approach [137139]. 
There exist models [140-146] in which one does not take into account all the effects (for example, microscopic selfconsistency of the density and nuclear potential) included in the TDHF method. In the framework of these models, one has attempted to describe in a unified manner the heavy-ion collisions in a wide range of energies, from the quasi-elastic to the deep-inelastic regime. The model [147-159] allows one to take explicitly into account the influence of the shell structure of nuclei on the collision dynamics. Constituent elements of the model are the realistic scheme of single-particle levels, nucleon separation energies, single-particle matrix elements of inelastic transitions of nucleons in the nuclei due to the effect of the changing field and the matrix elements of nucleon transfer from one nucleus to another. The singleparticle approximation is improved by the phenomenological inclusion of the residual interaction. The model also takes into account in a simpler manner the fluctuations associated with the distortion of the mean field in the framework of the random-phase approximation. The study of the evolution of single-particle degrees of freedom results in the transport coefficients that provides the microscopic basis for the phenomenological description of DIC with the master equation or Fokker-Planck equation [160]. Using the explicit equations for the single-particle operators, the transition probabilities between the macroscopic states of the system differing in charge (mass) asymmetry were obtained in [147-159].

\subsection{Microscopical description of MNT}

These models are based on the assumption that at low collision energies the DNS stage plays an important role. For the quantum-mechanical description of the internal degrees of freedom we use the single-particle approximation with realistic schemes of the single-particle levels for each nucleus. Qualitatively, the process is represented as follows: Two potential wells (Woods-Saxon potentials), whose centroids move on classical trajectories, perturb each other giving rise to the nucleon exchange and transitions. This mechanism is mainly responsible for the kinetic energy dissipation. Twonucleon collisions are indirectly taken into account through the Fermi (thermal) occupation numbers. The effects of residual interaction like excitation of high- and low-lying collective states are not explicitly taken into account, although the surface vibration modes contribute to dissipation. The adiabaticity of the relative motion with respect to these vibrations reduces this effect. It is convenient to represent the total Hamiltonian $H$ of the DNS in the form

$H=H_{r e l}+H_{0}+V_{\text {int }}$.

The Hamiltonian of the relative motion is the sum of the kinetic energy operator and the interaction potential $V(\mathbf{R})$ of the nuclei in the ground state:
$H_{\text {rel }}=\frac{\mathbf{P}^{2}}{2 \mu}+V(\mathbf{R})$.

Here, $\mathbf{R}$ is the distance between the centers of mass of two nuclei, $\mathbf{P}$ is the conjugate momentum and $\mu$ is the reduced mass of the system. The last two terms in (2) describe the internal motion of the non-interacting nuclei and the coupling of the relative motion to the internal degrees of freedom, respectively.

One can obtain from (2) the classical limit of the equations of motion for the macroscopic collective variables $R$ and $P$ :

$\dot{\mathbf{R}}=\nabla_{\mathbf{P}}\left(H_{\text {rel }}+\left\langle t\left|V_{\text {int }}\right| t\right\rangle\right)$,

$\dot{\mathbf{P}}=\nabla_{\mathbf{R}}\left(H_{\text {rel }}+\left\langle t\left|V_{\text {int }}\right| t\right\rangle\right)$,

where the average $\langle t|\cdots| t\rangle$ is over the internal variables at time $t$. The classical motion of the nuclei additionally depends on the time-dependent non-conservative coupling potential $\left\langle t\left|V_{\text {int }}\right| t\right\rangle$, for the calculation of which it is necessary to consider the equation of motion for the single-particle density matrix.

The single-particle basis is constructed from the asymptotic wave vectors of the single-particle states of the noninteracting nuclei- the projectile " $P$ ", $|P\rangle$, and the target nucleus " $T$ ", $|T\rangle$-in the form

$$
\begin{aligned}
& |\tilde{P}\rangle=|P\rangle-\frac{1}{2} \sum_{T}|T\rangle\langle T \mid P\rangle, \\
& |\tilde{T}\rangle=|T\rangle-\frac{1}{2} \sum_{P}|P\rangle\langle P \mid T\rangle .
\end{aligned}
$$

The orthogonality of this basis is satisfied up to the second order in $\langle P \mid T\rangle$. It is convenient to take the single-particle Hamiltonian as

$$
H(\mathbf{R}(t))=\sum_{i=1}^{A}\left(-\frac{\hbar^{2}}{2 m} \triangle_{i}+U_{P}\left(\mathbf{r}_{i}-\mathbf{R}(t)\right)+U_{T}\left(\mathbf{r}_{i}\right)\right),
$$

where $m$ is the nucleon mass, and $A=A_{P}+A_{T}$ is the total mass of the system. The mean single-particle potentials of the projectile, $U_{P}$, and of the target nucleus, $U_{T}$, include both, nuclear and Coulomb fields. In the second quantization representation, this Hamiltonian is written in the form

$$
\begin{aligned}
H(\mathbf{R}(t))= & H_{0}(\mathbf{R}(t))+V_{\mathrm{int}}(\mathbf{R}(t)), \\
H_{0}(\mathbf{R}(t))= & \sum_{P} \tilde{\varepsilon}_{P}(\mathbf{R}(t)) a_{P}^{+} a_{P}+\sum_{T} \tilde{\varepsilon}_{T}(\mathbf{R}(t)) a_{T}^{+} a_{T}, \\
V_{\mathrm{int}}(\mathbf{R}(t))= & \sum_{P \neq P^{\prime}} \chi_{P P^{\prime}}^{(T)}(\mathbf{R}(t)) a_{P}^{+} a_{P^{\prime}} \\
& +\sum_{T \neq T^{\prime}} \chi_{T T^{\prime}}^{(P)}(\mathbf{R}(t)) a_{T}^{+} a_{T^{\prime}} \\
& +\sum_{P, T} g_{P T}(\mathbf{R}(t))\left(a_{P}^{+} a_{T}+\text { h.c. }\right),
\end{aligned}
$$


where, up to the second order in $\langle P \mid T\rangle$,

$$
\begin{aligned}
& \tilde{\varepsilon}_{P}(\mathbf{R}(t))=\varepsilon_{P}+\left\langle P\left|U_{T}(\mathbf{r})\right| P\right\rangle, \\
& \tilde{\varepsilon}_{T}(\mathbf{R}(t))=\varepsilon_{T}+\left\langle T\left|U_{P}(\mathbf{r}-\mathbf{R}(t))\right| T\right\rangle, \\
& \chi_{P P^{\prime}}^{(T)}(\mathbf{R}(t))=\left\langle P\left|U_{T}(\mathbf{r})\right| P^{\prime}\right\rangle, \\
& \chi_{T T^{\prime}}^{(P)}(\mathbf{R}(t))=\left\langle T\left|U_{P}(\mathbf{r}-\mathbf{R}(t))\right| T^{\prime}\right\rangle, \\
& g_{P T}(\mathbf{R}(t))=\frac{1}{2}\left\langle P\left|U_{P}(\mathbf{r}-\mathbf{R}(t))+U_{T}(\mathbf{r})\right| T\right\rangle .
\end{aligned}
$$

Here, $\varepsilon_{P(T)}$ are the energies of the unperturbed single-particle states, which are characterized by the set of quantum numbers $P \equiv\left(n_{P}, j_{P}, l_{P}, m_{P}\right)\left[T \equiv\left(n_{T}, j_{T}, l_{T}, m_{T}\right)\right]$. The diagonal matrix elements $\left\langle P\left|U_{T}\right| P\right\rangle$ and $\left\langle T\left|U_{P}\right| T\right\rangle$ characterize the shifts of the energies of the single-particle levels due to the interaction of the nuclei. The non-diagonal matrix elements $\chi_{P P^{\prime}}^{(T)}$ and $\chi_{T T^{\prime}}^{(P)}$ correspond to particle-hole transitions between the levels in one of the nuclei under the influence of the mean field of the partner nucleus. In turn, the $g_{P T}$ determine the transitions of nucleons between the nuclei because of the action of the DNS mean field. The contributions to the matrix elements from non-inertial effects are not taken into account, since they are small [143].

Information about the evolution of the system can be obtained by solving the equation of motion for the singleparticle density matrix. However, the steady solution of the equation for the occupation numbers does not provide the Fermi thermal occupation numbers in the asymptotic limit $(t \rightarrow \infty)$ at fixed $R$, because the residual interaction between the nucleons has not been taken into account. In the reaction, there are simultaneously the excitation of nuclei and their relaxation to statistical equilibrium. Allowance for them in the Hamiltonian (2) leads to the appearance of the well-known two-particle collision term $[161,162]$ in the equations for the occupation numbers. Explicit consideration of the residual interaction requires extensive calculations. As shown in the time-dependent density-matrix theory [163], the two-body dissipation effects play an important role in fusion. For DIC, the fluctuations calculated from the Balian-Vénéroni variational principle [164] are much larger than standard TDHF results and closer to experimental fluctuations. One can take into account the twoparticle collision integral in linearized form ( $\tau$ approximation) $[165,166]$ :

$i \hbar \frac{\partial n(t)}{\partial t}=[H, n(t)]-\frac{i \hbar}{\tau}\left\{n(t)-n^{\mathrm{eq}}(\mathbf{R}(t))\right\}$,

where $\tau$ is the parameter of relaxation time, and $n^{\mathrm{eq}}(\mathbf{R}(t))$ is the local quasi-equilibrium distribution, i.e., the equilibrium distribution for a fixed value of $R$. The formal solution for $n_{i}(t)=\left\langle t\left|a_{i}^{+} a_{i}\right| t\right\rangle$ is as follows [167]:

$$
\begin{aligned}
n_{i}(t)= & \exp \left(\frac{t_{0}-t}{\tau}\right)\left\{n_{i}\left(t_{0}\right)\right. \\
& +\sum_{k} \int_{t_{0}}^{t} \mathrm{~d} t^{\prime} \int_{t_{0}}^{t^{\prime}} \mathrm{d} t^{\prime \prime} \Omega_{i k}\left(t^{\prime}, t^{\prime \prime}\right) \\
& \times \exp \left(\frac{t^{\prime \prime}-t}{\tau / 2}\right)\left[n_{k}\left(t^{\prime \prime}\right)-n_{i}\left(t^{\prime \prime}\right)\right] \\
& \left.+\frac{1}{\tau} \int_{t_{0}}^{t} \mathrm{~d} t^{\prime} n_{i}^{\mathrm{eq}}\left(\mathbf{R}\left(t^{\prime}\right)\right) \exp \left(\frac{t^{\prime}-t_{0}}{\tau}\right)\right\},
\end{aligned}
$$

where

$$
\begin{aligned}
\Omega_{i k}\left(t, t^{\prime}\right)= & \frac{2}{\hbar^{2}} \operatorname{Re}\left\{V_{i k}(\mathbf{R}(t)) V_{k i}\left(\mathbf{R}\left(t^{\prime}\right)\right)\right. \\
& \left.\times \exp \left[i \int_{t^{\prime}}^{t} \mathrm{~d} t^{\prime \prime} \tilde{\omega}_{k i}\left(\mathbf{R}\left(t^{\prime \prime}\right)\right)\right]\right\}
\end{aligned}
$$

and $\tilde{\omega}_{i k}=\left(\tilde{\varepsilon}_{i}-\tilde{\varepsilon}_{k}\right] / \hbar$. It is convenient to solve Eq. (10) iteratively, dividing the time interval $\left(t-t_{0}\right)$ into subintervals: $t_{0}, t_{0}+\Delta t, t_{0}+2 \Delta t$, etc. Then at times $\Delta t<\tau$, Eq. (10) is approximately written as

$$
\begin{aligned}
n_{i}(t)= & n_{i}^{\mathrm{eq}}(\mathbf{R}(t))\left[1-\exp \left(-\frac{\Delta t}{\tau}\right)\right] \\
& +\bar{n}_{i}(t) \exp \left(-\frac{\Delta t}{\tau}\right),
\end{aligned}
$$

where

$$
\begin{aligned}
\bar{n}_{i}(t)= & n_{i}(t-\Delta t) \\
& +\sum_{k} W_{i k}(\mathbf{R}(t), \Delta t)\left[n_{k}(t-\Delta t)-n_{i}(t-\Delta t)\right]
\end{aligned}
$$

and

$$
W_{i k}(\mathbf{R}(t), \Delta t)=\left|V_{i k}(\mathbf{R}(t))\right|^{2} \frac{\sin ^{2}\left(\frac{\Delta t}{2} \tilde{\omega}_{i k}(\mathbf{R}(t))\right)}{\left[\frac{\hbar}{2} \tilde{\omega}_{i k}(\mathbf{R}(t))\right]^{2}} .
$$

The dynamical $\left(n_{i}(t)\right)$ and quasi-equilibrium (i.e. thermal, $\left.n_{i}^{\text {eq }}(\mathbf{R}(t))\right)$ occupation numbers are calculated at each iterative step $\Delta t$. The parameters of the Fermi distribution for proton and neutron subsystems of each nucleus are determined by the conservation laws for energy and nucleon numbers of each type. The relative contributions of the single- and two-particle components are determined by the only parameter $\tau$ of our model. Knowing the values of the occupation numbers $n_{i}(t)$, we can readily determine at any time $t$ the mean numbers of protons, $Z_{P(T)}$ and neutrons, $N_{P(T)}$, and their variances $\sigma_{Z}^{2}$ or $\sigma_{N}^{2}$, and also find the fragment excitation energies $E_{P(T)}^{*}(t)$ : 


$$
\begin{aligned}
Z_{P(T)}(t)= & \sum_{P(T)}^{Z} n_{P(T)}(t), \\
N_{P(T)}(t)= & \sum_{P(T)}^{N} n_{P(T)}(t), \\
\sigma_{Z(N)}^{2}(t)= & \sum_{P}^{Z(N)} n_{P}(t)\left[1-n_{P}(t)\right], \\
E_{P(T)}^{*}(t)= & \sum_{P}\left[\tilde{\varepsilon}_{P(T)}(\mathbf{R}(t))\right. \\
& \left.-\varepsilon_{F_{P}\left(F_{T}\right)}(\mathbf{R}(t))\left[n_{P(T)}(t)-n_{P(T)}\left(t_{0}\right)\right]\right],
\end{aligned}
$$

where $\varepsilon_{F_{P}\left(F_{T}\right)}(\mathbf{R}(\mathbf{t}))$ is the Fermi energy of the light $(P)$ and heavy $(T)$ fragment. The upper indices, $Z$ and $N$, in the sum indicate the summation over the proton and neutron singleparticle levels, respectively. At each iterative step, we separate the contribution to the fragment excitation energy from the particle-hole excitations and from the exchange of nucleons.

As originally assumed, the kinetic energy of the relative motion, which is transformed into energy of internal excitation, is distributed between the fragments proportionally to their masses (thermodynamic equilibrium). However, it was shown later in a number of experiments that this assumption is not always correct. In some reactions, the excitation energy is distributed almost equally between the products of binary reactions [168-170]. In other reactions, the distribution of the excitation energy is between an equal distribution and one proportional to the masses of nuclei. In the reactions, where one nucleus is magic or close to magic, most of the excitation energy is concentrated in the complementary product at relatively large losses of kinetic energy [171,172].

The calculation of the friction forces requires the explicit treatment of the coupling of the relative motion to the internal motion [140-145,173]. The models differ in the internal excitations considered. They may be collective surface vibrations, giant resonances, incoherent particle-hole excitations, or transitions of nucleons between the nuclei. It is clear that the structure of the excited states of the nuclei and the coupling strength of various excitation modes to the relative motion influences the energy distribution between the fragments.

\subsection{The nucleus-nucleus potential}

The description of the DIC dynamics depends on the potential taken between the nuclei. In the phenomenological models, we discriminate between adiabatic and diabatic potentials. Adiabatic potentials represent the minimum of energy for a set of given collective coordinates and have an outside barrier, but in general no further inside barrier versus smaller internuclear distances. They allow for the formation

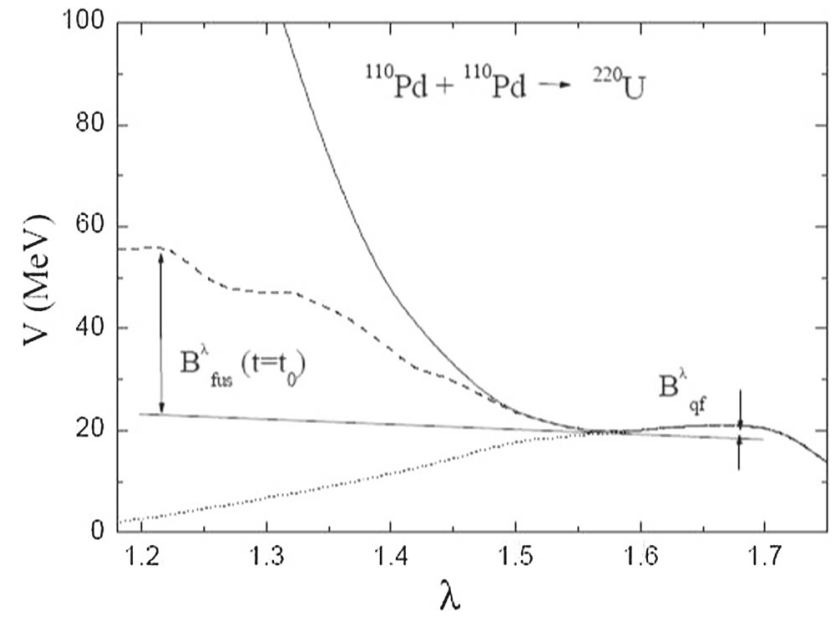

Fig. 10 The diabatic (solid curve), diabatic time-dependent (dashed curve) and adiabatic (dotted curve) nucleus-nucleus potentials for ${ }^{110} \mathrm{Pd}+{ }^{110} \mathrm{Pd}$ as a function of the coordinate $\lambda$ which measures the length of the system in units of the diameter of the spherical nucleus ${ }^{220} \mathrm{U}$ [175]. The heights of the fusion $\left(B_{f u s}^{\lambda}\right)$ and quasi-fission $\left(B_{q f}^{\lambda}\right)$ barriers are marked

of the compound system as a melting of two nuclei. Adiabatic potentials as a function of $\eta$ are usually smallest for symmetric fragmentations $(\eta=0)$ and therefore obtain the largest fusion cross-sections around $\eta=0$. Diabatic potentials include the effects of relative motion. At every avoided crossing of the single-particle energies in the adiabatic twocenter shell model the nucleons follow the so-called diabatic path, a process depending on the relative velocity of the nuclei and denoted as the Landau-Zener effect for which the Pauli principle between the nuclei is responsible. As an example, we show in Fig. 10 adiabatic and diabatic potentials for ${ }^{110} \mathrm{Pd}+{ }^{110} \mathrm{Pd}$ collisions as a function of the length of the nucleus-nucleus system [174,175]. As seen in Fig. 10, the time-dependent diabatic potential almost coincides with the phenomenological diabatic potential near touching configuration $(1.45<\lambda<1.7)$.

The phenomenological diabatic nucleus-nucleus interaction potential between two nuclei is written as

$$
\begin{aligned}
V(R, Z, N, J)= & V_{C}(R, Z, N)+V_{N}(R, Z, N) \\
& +V_{\text {rot }}(R, Z, N, J)
\end{aligned}
$$

which includes the Coulomb interaction, the nuclear interaction, and the centrifugal term. The centrifugal potential $V_{\text {rot }}$ in the formed DNS is usually expressed under the assumption that this system has the rigid body moment of inertia. In the calculation of the Coulomb and nuclear interactions, the deformations of the DNS nuclei are important. The Coulomb potential for two quadrupole deformed nuclei is analytically calculated following [176]. The nuclear part of the nucleus-nucleus potential can be obtained by using the double-folding formalism [177] with the density-dependent 
nucleon-nucleon interaction. The nucleus-nucleus potentials calculated with this $V_{\mathrm{N}}$ have a potential pocket resulting from the attractive nuclear and repulsive Coulomb interactions. With increasing Coulomb repulsion in the DNS the depth of the pocket decreases. For the pole-pole orientation of interacting nuclei, the potential pocket is situated at the distance $R_{\mathrm{m}}=R_{P}\left(1+\sqrt{5 /(4 \pi)} \beta_{P}\right)+R_{T}\left(1+\sqrt{5 /(4 \pi)} \beta_{T}\right)+$ $0.5 \mathrm{fm}\left(\beta_{P}\right.$ and $\beta_{T}$ are the quadrupole deformation parameters of the nuclei with radii $R_{P}$ and $R_{T}$ ) and keeps the DNS nuclei in contact. The value of $B_{\mathrm{qf}}(Z, N)$ coincides with the depth of this pocket and decreases with increasing $Z$ and $J$. For $J \leq 30$, the dependence of $B_{\mathrm{qf}}(Z, N)$ is weak and can be disregarded. The decaying DNS with given $Z$ and $N$ has to overcome the potential barrier in $R$ at $R_{\mathrm{b}}=R_{\mathrm{m}}+1.5 \mathrm{fm}$ on the potential-energy surface. The radius of a nucleus is parameterized as $R_{P(T)}=r_{0} A_{P(T)}^{1 / 3}$, where $r_{0}=1.15-1.16 \mathrm{fm}$.

The density-constrained along with the TDHF was used in [178-181] to obtain the nucleus-nucleus potentials

$V(R)=E_{\mathrm{DC}}-E_{P}-E_{T}$,

where $E_{\mathrm{DC}}$ is the density-constrained energy at the instantaneous separation $R(t)$, while $E_{P}$ and $E_{T}$ are the binding energies of the two nuclei obtained with the same effective interaction. The concept of an adiabatic reference state for a given TDHF state is used in Eq. (20). The calculation of the excitation energy is achieved by dividing the TDHF motion into a collective and intrinsic part [182]. The major assumption is that the collective part is determined by the density $\rho(\mathbf{r}, t)$ and current $\mathbf{j}(\mathbf{r}, t)$. Consequently, the excitation energy is written as

$E^{*}(t)=E_{\mathrm{TDHF}}-E_{\text {coll }}(\rho(t), \mathbf{j}(t))$,

where $E_{\mathrm{TDHF}}$ is the total energy of the dynamical system and $E_{\text {coll }}=E_{\text {kin }}(\rho(t), \mathbf{j}(t))+E_{D C}(\rho(t))$ represents the collective energy of the system. Here, $E_{\text {kin }}(\rho(t), \mathbf{j}(t))=$ $\frac{m}{2} \int \mathrm{d} \mathbf{r} \mathbf{j}^{2}(t) / \rho(t)$ is the kinetic part which is asymptotically equivalent to the kinetic energy $\frac{1}{2} \mu \dot{R}^{2}$ of the relative motion of two nuclei. The calculated capture cross-sections with this $V(R)$ are found to be in a reasonable agreement with data and other model calculations.

\subsection{Transport coefficients}

Using (4), we obtain the integro-differential equation

$$
\begin{aligned}
\frac{\mathrm{d}}{\mathrm{d} t}[\mu(\mathbf{R}(t)) \dot{\mathbf{R}}(t)]= & -\nabla_{\mathbf{R}} V(\mathbf{R}(t)) \\
& -2 \operatorname{Re} \sum_{i \neq k} \nabla_{\mathbf{R}} V_{i k}(\mathbf{R}(t)) n_{i k}(t) .
\end{aligned}
$$

The second term on the right-hand side contains effective forces, of both, conservative and dissipative nature, due to the coupling of the relative motion with the internal motion. This term depends explicitly not only on the relative separation $R(t)$ but also on the current time $t$, in contrast to the linearresponse theory [126] in which the asymptotic values are taken. In the adiabatic limit, when the characteristic time of the collective motion is much larger than the relaxation time, we can expand the function $V_{i k}\left(\mathbf{R}\left(t^{\prime}\right)\right)$ in Eq. (22) near the point $t^{\prime}=t$ :

$$
\begin{aligned}
V_{i k}\left(\mathbf{R}\left(t^{\prime}\right)\right)= & V_{i k}(\mathbf{R}(t))+\left(t^{\prime}-t\right) \dot{\mathbf{R}}(t) \nabla_{\mathbf{R}} V_{i k}(\mathbf{R}(t)) \\
& +\frac{\left(t^{\prime}-t\right)^{2}}{2} \ddot{\mathbf{R}}(t) \nabla_{\mathbf{R}} V_{i k}(\mathbf{R}(t)) \\
& +\frac{\left(t^{\prime}-t\right)^{2}}{2}\left(\dot{\mathbf{R}}(t) \nabla_{\mathbf{R}}\right)^{2} V_{i k}(\mathbf{R}(t))+\cdots
\end{aligned}
$$

The first term of this expansion gives the dynamical correction

$$
\begin{aligned}
-\nabla_{\mathbf{R}} \delta V(\mathbf{R}(t))= & \sum_{i k} V_{i k}(\mathbf{R}(t)) \nabla_{\mathbf{R}} V_{k i}(\mathbf{R}(t)) \\
& \times B_{i k}^{(0)}\left(t, t_{0}\right)\left[n_{i}(t)-n_{k}(t)\right]
\end{aligned}
$$

to the conservative force. Here,

$$
\begin{aligned}
B_{i k}^{(n)}\left(t, t_{0}\right)= & \frac{2}{\hbar} \int_{t_{0}}^{t} \mathrm{~d} t^{\prime} \frac{\left(t^{\prime}-t\right)^{n}}{n !} \exp \left(\frac{t^{\prime}-t}{\tau / 2}\right) \\
& \times \sin \left\{\tilde{\omega}_{k i}(\mathbf{R}(t))\left[t-t^{\prime}\right]\right\} .
\end{aligned}
$$

One can similarly write down the correction

$$
\begin{aligned}
\delta V(\mathbf{R}(t))= & \frac{1}{2} \sum_{i k}\left|V_{i k}(\mathbf{R}(t))\right|^{2} B_{i k}^{(0)}\left(t, t_{0}\right) \\
& \times\left[n_{k}(t)-n_{i}(t)\right]
\end{aligned}
$$

to the conservative potential $V(\mathbf{R}(t))$ due to the particle-hole excitations and the exchange of nucleons.

The second term in Eq. (23) leads to an irreversible loss of kinetic energy of the relative motion, which is transformed into internal excitation of the nuclei. The coefficients of radial and tangential friction, respectively, are written in the form

$$
\begin{aligned}
k_{r}(\mathbf{R}(t))= & \sum_{i k}\left|\frac{\partial V_{i k}(\mathbf{R}(t))}{\partial R}\right|_{i k}^{2}\left(t, t_{0}\right)\left[n_{k}(t)-n_{i}(t)\right], \\
k_{t}(\mathbf{R}(t))= & \frac{1}{2(R(t))^{2}} \sum_{i k, L} L(L+1)\left|V_{i k}^{L}(\mathbf{R}(t))\right|^{2} \\
& \times B_{i k}^{(1)}\left(t, t_{0}\right)\left[n_{k}(t)-n_{i}(t)\right],
\end{aligned}
$$

where

$$
V_{i k}(\mathbf{R}(t))=\sum_{L=\left|l_{k}-l_{i}\right|}^{l_{k}+l_{i}} V_{i k}^{L}(\mathbf{R}(t))
$$

and $V_{i k}^{L}(\mathbf{R}(t))$ is the partial form factor for the given angularmomentum transfer $L[157,158]$. 
The third term in Eq. (23) results in the correction

$$
\begin{aligned}
\Delta \mu(\mathbf{R}(t))= & 2 \sum_{i k}\left|\nabla_{\mathbf{R}} V_{i k}(\mathbf{R}(t))\right|^{2} B_{i k}^{(2)}\left(t, t_{0}\right) \\
& \times\left[n_{k}(t)-n_{i}(t)\right]
\end{aligned}
$$

to the reduced mass $\mu(\mathbf{R}(t))$. The transport coefficients can be similarly calculated for other collective variables such as the mass (charge) asymmetry and the deformation of the nuclei of the DNS.

Using the macroscopic reduction procedure of the meanfield theory proposed in Ref. [183], the friction coefficient associated with one-body energy dissipation was extracted in the entrance channel of heavy-ion fusion reactions [184]. The magnitude and form factor of the reduced friction coefficient have a universal property for various reactions investigated and are in a good agreement with those calculated with Eq. (28). The nucleus-nucleus potentials obtained with the density-constrained TDHF method exhibit energy dependence [183] and account for dynamical effects. The magnitude and form factor of the extracted friction coefficient depend on the beam energy as well. At low energies, nuclear dissipation is dominated by the one-body dissipation mechanism (nucleon exchange plus excitation of surface modes) based on the mean-field dynamics.

In the stochastic mean-field approach [185], the meanfield fluctuations are incorporated into the dynamical evolution by including the initial state fluctuations in a stochastic approximation [186-189]. The diffusion coefficient for nucleon exchange is extracted in a semiclassical approximation and has a form similar to that in the phenomenological nucleon exchange model. Using this similarity, one also infers an expression for the nucleon drift coefficient in the semiclassical approximation. While the diffusion coefficient is insensitive to the neck motion, the nucleon drift coefficient is affected by this motion.

\subsection{Excitation energy distributions, centroids of charge and mass distributions}

To demonstrate the possibilities of the model presented, we show the calculated distribution of excitation energy between the products of deep inelastic heavy-ion collisions in the ${ }^{56} \mathrm{Fe}(505 \mathrm{MeV})+{ }^{165}$ Ho reaction [190-192]. The results for other reactions can be found in Refs. [167,193]. The theoretical and experimental results for the ratio $R_{P}=$ $E_{P}^{*} /\left(E_{P}^{*}+E_{T}^{*}\right)$ of excitation energy of the projectile-like nucleus to the total excitation energy of the DNS are shown in Fig. 11. We present the relative contributions of nucleon exchange and particle-hole excitations in the process of dissipation of the kinetic energy of relative motion. The calculated ratios $R_{P}^{(\mathrm{ex})}=E_{P}^{*(\mathrm{ex})} /\left(E_{P}^{*(\mathrm{ex})}+E_{T}^{*(\mathrm{ex})}\right)$ for nucleon exchange and $R_{P}^{(\mathrm{ph})}=E_{P}^{*(\mathrm{ph})} /\left(E_{P}^{*(\mathrm{ph})}+E_{T}^{*(\mathrm{ph})}\right)$ for particle-

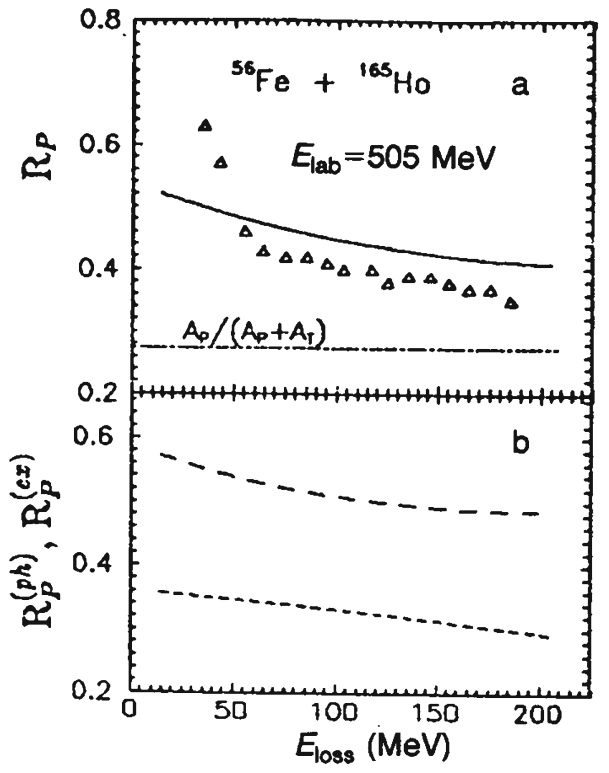

Fig. 11 a Ratio $R_{P}$ of the excitation energy $\left(E_{P}^{*}\right)$ of the projectile-like fragment to the total excitation energy $E_{\text {loss }}=E_{P}^{*}+E_{T}^{*}$ of the system as a function of $E_{\text {loss }}$ for the ${ }^{56} \mathrm{Fe}(505 \mathrm{MeV})+{ }^{165}$ Ho reaction. The triangles represent experimental results [191]. The results of the model calculations [193] are shown by the solid curve. The dash-dotted curve marks the limit of thermal equilibrium $\left[E_{P}^{*} / E_{\text {loss }}^{*}=A_{P} /\left(A_{P}+A_{T}\right)\right]$. b Dependencies of the calculated ratios $R_{P}^{(\mathrm{ex})}$ (dashed curve) and $R_{P}^{(\mathrm{ph})}$ (dotted curve) for the same reaction

hole excitations are shown in Fig. 11. Here, $E_{P(T)}^{*(\mathrm{ex})}$ and $E_{P(T)}^{*(\mathrm{ph})}$ are the contributions to the total excitation $E_{P(T)}^{*}$ from nucleon exchange and from particle-hole excitations, respectively. The theoretical ratio of the excitation energy $E_{\text {loss }}^{(p h)}=E_{P}^{*(\mathrm{ph})}+E_{T}^{*(\mathrm{ph})}$ associated with the particle-hole excitations to the energy $E_{\text {loss }}^{(\mathrm{ex})}=E_{P}^{*(\mathrm{ex})}+E_{T}^{*(\mathrm{ex})}$ due to nucleon exchange varies from 0.2 to 0.7 in all reactions considered [167,193]. As shown, in the reactions with ${ }^{58} \mathrm{Ni}$, ${ }^{74} \mathrm{Ge}$, and ${ }^{56} \mathrm{Fe}$ the nucleon exchange plays the main role, $E_{\text {loss }}^{(\mathrm{ph})} / E_{\text {loss }}^{(\mathrm{ex})} \approx 0.2-0.3$, in the dissipation of the kinetic energy. In the reactions with heavier nuclei, the importance of particle-hole mechanism increases, $E_{\text {loss }}^{(\mathrm{ph})} / E_{\text {loss }}^{(\mathrm{ex})} \approx$ $0.6-0.7$.

The mean values of the charge (mass) distributions of the reaction products are also well described with the single-particle matrix elements (8) analytically determined as in Refs. [157,158,160,167]. The calculations showed that variation of the only free parameter $\tau$ from $5 \times$ $10^{-23} \mathrm{~s}$ to $25 \times 10^{-22} \mathrm{~s}$ does not affect significantly the results.

Similar conclusions on the dynamics of DIC have been drawn with other theoretical approaches. Applying the improved quantum molecular dynamics (ImQMD) model [194], the energy dependencies of the nucleus-nucleus potential, the friction parameter, and the random force char- 
acterizing a one-dimensional Langevin-type description of the heavy-ion fusion process were investigated [195, 196]. As shown microscopically, the energy dissipation in the fusion process is governed by two mechanisms: One is caused by the nucleon exchanges between two fusing nuclei, and the other is due to a rearrangement of nucleons in the intrinsic system. The former mechanism monotonically increases the dissipative energy and shows a weak dependence on the incident energy, while the latter depends on both the relative distance between two fusing nuclei and the incident energy. As shown, the latter mechanism is responsible for the energy dependence of the fusion potential. The ImQMD model can be also used to predict the isotopic production cross-sections in multinucleon transfer reactions. While the GRAZING model [197-200] is suitable to estimate the production cross-sections as a result of the transfer of several nucleons, the approach based on the ImQMD model [40] provides wider isotopic distributions and is suitable for describing the transfer products rather far from the entrance channel.

The competition between collective motion and singleparticle degrees of freedom in dissipative processes was demonstrated in Ref. [201]. The spin-orbit force causes a significant enhancement of the dissipation in DIC. The theoretical fusion cross-section shows reasonably good agreement with the experimental data, considering that no free parameters are adjusted to reaction dynamics in the TDHF approach.

\subsection{Master equations for nucleon transfer}

To describe the mass (charge) distributions in DIC and QF reactions, and the fusion probability, one can apply master equations for the transfer of nucleons between the parts of the DNS $[20,167,202]$. As coordinates we choose the charge, neutron and mass numbers of both nuclei of the dinuclear system, namely $Z_{P}=Z, N_{P}=N$ and $A_{P}=Z+N$ for the projectile-like (light) nucleus and $Z_{T}=Z_{\text {tot }}-Z_{P}$, $N_{T}=N_{\text {tot }}-N_{P}$ and $A_{T}=A_{\text {tot }}-A_{P}$ for the target-like (heavy) nucleus. For the derivation of the master equations for the probability $P_{Z, N}(t)$ to find the DNS in the state $(Z, N)$ at time $t$, we start with the Hamiltonian (2) of the DNS at $R=R_{m}$ and finally obtain [167]

$$
\begin{aligned}
\frac{\mathrm{d}}{\mathrm{d} t} & P_{Z, N}(t) \\
= & \Delta_{Z+1, N}^{(-, 0)} P_{Z+1, N}(t)+\Delta_{Z-1, N}^{(+, 0)} P_{Z-1, N}(t) \\
& +\Delta_{Z, N+1}^{(0,-)} P_{Z, N+1}(t)+\Delta_{Z, N-1}^{(0,+)} P_{Z, N-1}(t) \\
& -\left(\Delta_{Z, N}^{(-, 0)}+\Delta_{Z, N}^{(+, 0)}+\Delta_{Z, N}^{(0,-)}+\Delta_{Z, N}^{(0,+)}\right) P_{Z, N}(t) \\
& -\left(\Lambda_{Z, N}^{q f}+\Lambda_{Z, N}^{f i s}\right) P_{Z, N}(t)
\end{aligned}
$$

with the transition rates

$$
\begin{aligned}
\Delta_{Z, N}^{( \pm, 0)}(T)= & \frac{1}{\Delta t} \sum_{P, T}^{Z}\left|g_{P T}\right|^{2} n_{P}(T)\left(1-n_{P}(T)\right) \\
& \times \frac{\sin ^{2}\left(\Delta t\left(\tilde{\varepsilon}_{P}-\tilde{\varepsilon}_{T}\right) / 2 \hbar\right)}{\left(\tilde{\varepsilon}_{P}-\tilde{\varepsilon}_{T}\right)^{2} / 4}, \\
\Delta_{Z, N}^{(0, \pm)}(T)= & \frac{1}{\Delta t} \sum_{P, T}^{N}\left|g_{P T}\right|^{2} n_{T}(T)\left(1-n_{P}(T)\right) \\
& \times \frac{\sin ^{2}\left(\Delta t\left(\tilde{\varepsilon}_{P}-\tilde{\varepsilon}_{T}\right) / 2 \hbar\right)}{\left(\tilde{\varepsilon}_{P}-\tilde{\varepsilon}_{T}\right)^{2} / 4},
\end{aligned}
$$

where the temperature-dependent Fermi occupation numbers for the single-particle states are used. The last line in (30) takes into account the DNS decay in $R\left(\Lambda_{Z, N}^{\mathrm{qf}}\right)$ and possible fission $\left(\Lambda_{Z, N}^{\mathrm{fis}}\right)$ of the heavy nucleus in the DNS. The initial condition for the master equations is $P_{Z, N}(0)=\delta_{Z, Z_{i}} \delta_{N, N_{i}}$.

The stability of the DNS with $\left(Z^{\prime}, N^{\prime}\right)$ is determined not only by the local minima of $\Delta_{Z, N}^{( \pm, 0)}$ and $\Delta_{Z, N}^{(0, \pm)}$ but also by the condition $\Delta_{Z, N}^{(+, 0)}>\Delta_{Z, N}^{(-, 0)}\left(\Delta_{Z, N}^{(0,+)}>\Delta_{Z, N}^{(0,-)}\right)$ at $Z<Z^{\prime}$ $\left(N<N^{\prime}\right)$ and $\Delta_{Z, N}^{(+, 0)}<\Delta_{Z, N}^{(-, 0)}\left(\Delta_{Z, N}^{(0,+)}<\Delta_{Z, N}^{(0,-)}\right)$ for $Z>$ $Z^{\prime}\left(N>N^{\prime}\right)$. So, the DNS evolution in mass asymmetry depends on the structure of the two nuclei. With increasing temperature of the system, the influence of the shell effects on the nucleon transfer decreases. This is reflected in a certain decrease of the oscillations of $\Delta_{Z, N}^{( \pm, 0)}\left(\Delta_{Z, N}^{(0, \pm)}\right)$ with varying $Z(N)$ when the temperature increases [167].

The transition rates $\Delta_{Z, N}^{( \pm, 0)}$ and $\Delta_{Z, N}^{(0, \pm)}$ depend on the temperature only through the Fermi occupation numbers. In the case of strongly asymmetric DNS configurations, the separation between the single-particle levels in the light fragment is larger than the temperature $T$ in the considered region of excitation energies, i.e., the deviation of occupation numbers from 1 or 0 is small, and the shell effects are manifested clearer. For nearly symmetric configurations, the change in the fragment Fermi energies $\Delta \tilde{\varepsilon}_{F_{P}, F_{T}}^{Z}\left(\Delta \tilde{\varepsilon}_{F_{P}, F_{T}}^{N}\right)$ on the transition $Z \rightarrow Z+1(N \rightarrow N+1)$ is less than $T$, the main contribution to the sums in (31) is made by the terms with $n_{T}^{Z}(T) \approx n_{P}^{Z}(T)$ and $\Delta \tilde{\varepsilon}_{F_{T}}^{Z} \approx-\Delta \tilde{\varepsilon}_{F_{P}}^{Z}$ and therefore

$\Delta_{Z, N}^{(-, 0)}-\Delta_{Z+1, N}^{(-, 0)} \sim \frac{\Delta \varepsilon_{F_{T}}^{Z}}{T}$.

Similar conclusions follow from consideration of the differences $\Delta_{Z, N}^{(+, 0)}-\Delta_{Z+1, N}^{(+, 0)}, \Delta_{Z, N}^{(0,-)}-\Delta_{Z, N+1}^{(0,-)}$, and $\Delta_{Z, N}^{(0,+)}-$ $\Delta_{Z, N+1}^{(0,+)}$. So, the weakening of the influence of shell structure on the nucleon transfer with increasing $T$ occurs slower than the exponential decrease of the shell correction to the binding energy of the nuclei. One can approximately express the ratio, for example $\Delta_{Z+1, N}^{(-, 0)} / \Delta_{Z, N}^{(+, 0)}$, through the ratio of corresponding level densities $\rho_{Z, N} / \rho_{Z+1, N}$. Though this approximation firstly suggested in Ref. [123] and later used in Refs. [31,33, 34, 203] washes out the structure peculiarities 
in the dynamics, it considerably simplifies the calculations. Indeed, only the multidimensional potential-energy surface is required to calculate the transition rates. The master equations (30) can be spread to other collective variables besides $Z$ and $N$. The evolution in the phase space can be also considered with the Langevin equations. Calculating many classical trajectories with the defined transition rates and random forces, the distribution in collective variables is obtained as in the solution of master equations.

The decay rates of the DNS in $R$ are treated with the onedimensional Kramers rate

$$
\begin{aligned}
\Lambda_{Z, N}^{\mathrm{qf}}(T)= & \frac{\omega}{2 \pi \omega^{B_{\mathrm{qf}}^{R}}}\left(\sqrt{\left(\frac{\Gamma}{2 \hbar}\right)^{2}+\left(\omega^{B_{\mathrm{qf}}^{R}}\right)^{2}}-\frac{\Gamma}{2 \hbar}\right) \\
& \times \exp \left(-\frac{B_{\mathrm{qf}}^{R}(Z, N)}{T(Z, N)}\right) .
\end{aligned}
$$

They depend on the height $B_{\mathrm{qf}}^{R}$ of the outer potential barrier at the internuclear distance $R_{\mathrm{b}}$ which is nearly independent of the angular momentum at $J<70$ if the DNS has a large moment of inertia. The height of the barrier is about $4.5 \mathrm{MeV}$ for $Z=20$ and less than $0.5 \mathrm{MeV}$ for $Z=Z_{\text {tot }} / 2 \pm 10$. The temperature is obtained with the Fermigas expression $T=\left(E^{*} / a\right)^{1 / 2} \mathrm{MeV}$ by using the excitation energy $E^{*}(Z, N)$ of the DNS and $a=A_{\text {tot }} / 12 \mathrm{MeV}^{-1}$. For a nearly symmetric DNS we have about $T=1.5 \mathrm{MeV}$. The potential is approximated by an inverted harmonic oscillator with the frequency $\omega^{B_{\mathrm{qf}}^{R}}$ around the top of the barrier and by a harmonic oscillator with frequency $\omega$ at the pocket. We use constant values for these quantities: $\hbar \omega^{B_{\mathrm{qf}}^{R}}=1.0 \mathrm{MeV}$, $\hbar \omega=2.0 \mathrm{MeV}$, and we set the width $\Gamma=2.8 \mathrm{MeV}$.

The mass and charge yields are then obtained:

$Y_{Z, N}\left(t_{0}\right)=\Lambda_{Z, N}^{q f} \int_{0}^{t_{0}} P_{Z, N}(t) \mathrm{d} t$.

The reaction time $t_{0}$ is determined by the balance equation of the probabilities:

$$
\begin{aligned}
& \sum_{Z, N}\left(\Lambda_{Z, N}^{\mathrm{qf}}+\Lambda_{Z_{\mathrm{tot}}-Z, N_{\mathrm{tot}}-N}^{\mathrm{fis}}\right) \int_{0}^{t_{0}} P_{Z, N}(t) \mathrm{d} t \\
& \quad=1-P_{\mathrm{CN}}
\end{aligned}
$$

with the fusion probability $P_{\mathrm{CN}}$

$$
P_{C N}=\sum_{Z>Z_{\mathrm{BG}}, N>N_{\mathrm{BG}}} P_{Z, N}\left(t_{0}\right),
$$

where $Z_{\mathrm{BG}}$ and $N_{B G}$ are determined by the barrier for fusion in the mass (charge) asymmetry coordinates. The DNS with $Z>Z_{\mathrm{BG}}$ evolves to the compound nucleus in a time less than $10^{-20} \mathrm{~s}$ which is much shorter than the decay time of the compound nucleus. For small angular momenta related to the $\mathrm{QF}$ reactions, $t_{0} \approx(3-4) \times 10^{-20} \mathrm{~s}$, while in DIC being at large angular momenta the value of $t_{0}$ is about ten times smaller. So, the model presented suggest us one way to describe the yield of products of DIC and QF reactions. Indeed, the mechanisms of these processes are similar but differ in time scale. The calculated angular distributions of the cross-sections in Ref. [204], together with the observed experiment-theory agreement indicate that the production of exotic nuclei in DIC at near-barrier energies peaks at 0 degree. Their production is associated, in particular, with the removal of protons and transfer of neutrons. The differential reaction cross-sections seem to be maximal at 0 degrees for the most exotic fragments.

The interaction time is considerably reduced in the reactions with two heavy nuclei where the value of $B_{\mathrm{qf}}^{R}$ is almost zero. Stable beams as ${ }^{136} \mathrm{Xe}$ or ${ }^{192} \mathrm{Os}$ and ${ }^{208} \mathrm{~Pb}$ target were suggested in Ref. [31] for producing neutron-rich nuclei with neutron number close to 126 (important for astrophysics area of the last waiting point in the $r$-process nucleosynthesis) at energies about 20-30 $\mathrm{MeV}$ higher than the corresponding Coulomb barriers. In these reactions, the transfer of several protons from target to projectile has relatively high probability because of small $Q$ value. However, the short interaction time due to the strong Coulomb repulsion could reduce the yield of transfer products. In the calculations, longer interaction time is achieved with larger friction coefficient corresponding to $\Gamma>3 \mathrm{MeV}$. However, the friction strength can not considerably exceed the estimate resulting from the TDHF calculations $[183,183,184]$ and analysis of the experimental data on the kinetic energy dissipation. In Ref. [33], the neutron and proton transfer rates scaling factor is the crucial parameter. Indeed, its increase by a factor 2 leads to more than 10 times larger yield of neutron-rich products. The value of the nucleon transfer rate was taken to describe the known experimental data. As proposed, in the ${ }^{238} \mathrm{U}+{ }^{248} \mathrm{Cm}$ reaction at $E_{\mathrm{c} . \mathrm{m} .}=770 \mathrm{MeV}$ one can produce new neutron-rich isotopes of elements up to $\mathrm{Sg}$ with the cross-sections larger than $1 \mathrm{pb}$.

The mass and charge yields of products are given by

$$
Y\left(A_{P}\right)=\sum_{Z} Y_{Z, A_{P}-Z}\left(t_{0}\right), \quad Y\left(Z_{P}\right)=\sum_{N} Y_{Z, N}\left(t_{0}\right) .
$$

The partial and total cross-section for quasi-fission can be calculated as:

$$
\begin{aligned}
\sigma_{\mathrm{qf}}\left(E_{\mathrm{c} . \mathrm{m} .}, A_{P}\right) & =Y\left(A_{P}\right) \sigma_{\mathrm{cap}}\left(E_{\mathrm{c} . \mathrm{m} .}\right), \\
\sigma_{\mathrm{qf}}\left(E_{\mathrm{c} . \mathrm{m} .}\right) & =\sum_{A_{P}} \sigma_{\mathrm{qf}}\left(E_{\text {c.m. }}, A_{P}\right) .
\end{aligned}
$$

The fission probability of the heavier nucleus is defined as

$$
P_{f}=\sum_{Z, N} \Lambda_{Z_{\mathrm{tot}}-Z, N_{\mathrm{tot}}-N}^{\mathrm{fis}} \int_{0}^{t_{0}} P_{Z, N}(t) \mathrm{d} t
$$


The capture cross-section, given as

$\sigma_{\text {cap }}\left(E_{\text {c.m. }}\right)=\frac{\pi \hbar^{2}}{2 \mu E_{\text {c.m. }}} J_{\text {cap }}\left(J_{\text {cap }}+1\right)$,

which depends on $J_{\text {cap }} \leq\left(2 \mu R_{b}^{2}\left(E_{\text {c.m. }}-V_{b}\right) / \hbar^{2}\right)^{1 / 2}=J_{\max }^{\text {kin }}$ (the kinematic angular momentum), which is smaller than the critical angular momentum $J_{\text {crit }}$. Trajectories with $J \geq J_{\text {crit }}$ contribute to deep-inelastic and quasi-elastic collisions.

To explain the experimental TKE of the QF products, one has to regard the large polarizations of the DNS nuclei. For nearly symmetric dinuclear systems with $\left(A_{P}+A_{T}\right) / 2-$ $20 \leq A_{P} \leq\left(A_{P}+A_{T}\right) / 2+20$ we found deformations which are about 3-4 times larger than the deformations of the nuclei in their ground states. Let us assume the distribution of the fragments in charge, mass and deformation to be

$$
\begin{aligned}
W\left(Z, N, \beta_{P}, \beta_{T}\right)= & Y_{Z, N} w_{\beta_{P}}(Z, N) \\
& \times w_{\beta_{T}}\left(Z_{\text {tot }}-Z, N_{\text {tot }}-N\right),
\end{aligned}
$$

where we set the distributions of the deformations $\beta_{P}$ and $\beta_{T}$ as Gaussian distributions

$w_{\beta}(Z, N)=\frac{1}{\sqrt{2 \pi \sigma_{\beta}^{2}}} \exp \left(-(\beta-\langle\beta\rangle)^{2} /\left(2 \sigma_{\beta}^{2}\right)\right)$.

Here, $\sigma_{\beta}^{2}=\left(\hbar \omega_{\mathrm{vib}} /\left(2 C_{\mathrm{vib}}\right)\right) \operatorname{coth}\left(\hbar \omega_{\mathrm{vib}} /(2 k T)\right)$ with the frequency $\omega_{\mathrm{vib}}(Z, N)$ and the stiffness parameter $C_{\mathrm{vib}}(Z, N)$ of the quadrupole vibrations are determined from experimental spectra. The average TKE is obtained as a function of the mass number $A_{P}$ of the light fragment

$$
\left\langle\operatorname{TKE}\left(A_{P}\right)\right\rangle=\frac{\iint \mathrm{d} \beta_{P} \mathrm{~d} \beta_{T} \sum_{\substack{Z, N \\ Z+N=A_{P}}} T K E \times W}{\iint \mathrm{d} \beta_{P} \mathrm{~d} \beta_{T} \sum_{\substack{Z, N \\ Z+N=A_{P}}} W}
$$

with TKE $=V\left(R_{b}\right)$. The variance of the TKE is

$$
\begin{aligned}
& \left.\sigma_{\mathrm{TKE}}^{2} \approx \sum_{Z} \operatorname{TKE}^{2}\right|_{\substack{\beta_{P}=\left\langle\beta_{P}\right\rangle \\
\beta_{T}=\left\langle\beta_{T}\right\rangle}} \frac{Y_{Z, A_{P}-Z}\left(t_{0}\right)}{\sum_{Z} Y_{Z, A_{P}-Z}\left(t_{0}\right)} \\
& -\left\langle\operatorname{TKE}\left(A_{P}\right)\right\rangle^{2}+\left(\sigma_{\mathrm{TKE}}^{\mathrm{def}}\left(A_{P}\right)\right)_{P}^{2}+\left(\sigma_{\mathrm{TKE}}^{\mathrm{def}}\left(A_{P}\right)\right)_{T}^{2}
\end{aligned}
$$

with $(j=P, T)$

$$
\begin{aligned}
\left(\sigma_{\mathrm{TKE}}^{\mathrm{def}}\left(A_{P}\right)\right)_{j}^{2}= & \left.\sum_{Z}\left(\frac{\partial T K E}{\partial \beta_{j}}\right)^{2}\right|_{\substack{\beta_{P}=\left\langle\beta_{P}\right\rangle \\
\beta_{T}=\left\langle\beta_{T}\right\rangle}} \\
& \times \frac{\sigma_{\beta_{j}}^{2} Y_{Z, A_{P}-Z}\left(t_{0}\right)}{\sum_{Z} Y_{Z, A_{P}-Z}\left(t_{0}\right)} .
\end{aligned}
$$

As examples, we show here the charge distributions calculated in the reactions ${ }^{238} \mathrm{U}+{ }^{40} \mathrm{Ca}(340 \mathrm{MeV})$ and ${ }^{238} \mathrm{U}+{ }^{48} \mathrm{Ca}$ (425 MeV) (Fig. 12). The calculations were performed for interaction time $t_{0}=2 \times 10^{-21} \mathrm{~s}$ and $T=1.5 \mathrm{MeV}$ [205]. These parameters correspond to the collisions with the angular momenta close to $J_{\text {crit }}$ being characteristic of DIC. The

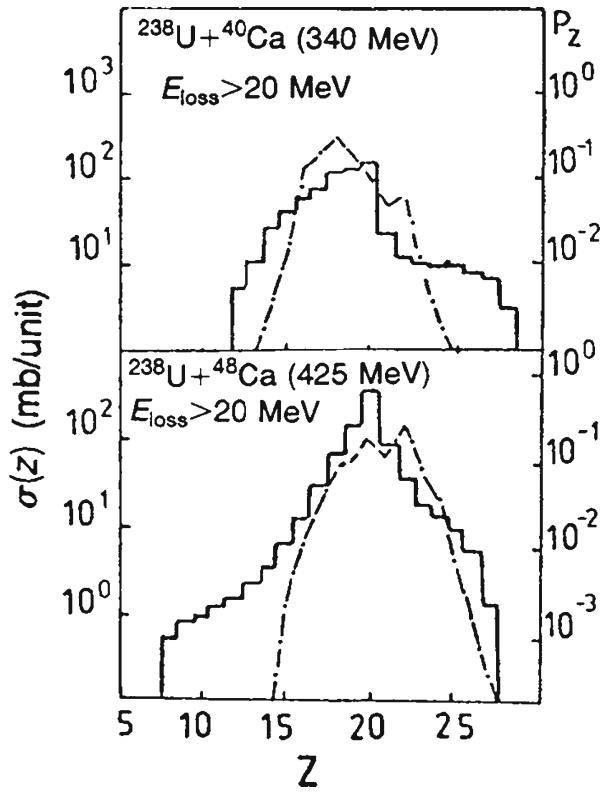

Fig. 12 Charge distributions $P_{Z}$ of the products of the reactions ${ }^{238} \mathrm{U}+{ }^{40} \mathrm{Ca}(340 \mathrm{MeV})$ and ${ }^{238} \mathrm{U}+{ }^{48} \mathrm{Ca}(425 \mathrm{MeV})$ calculated for $t_{0}=2 \times 10^{-21} \mathrm{~s}$ and $T=1.5 \mathrm{MeV}$ (dot-dashed lines) [167]. The solid lines show the experimental values of the cross-sections [64]

theoretical results agree well with the experimental ones near the maxima of distributions. The underestimation of the tails of distributions is due to the contribution of collisions with $J$ leading to longer interaction times.

\section{6 $Q_{g g}$ systematics}

As established experimentally [57], the cross-sections for production of the isotopes of nuclei with $Z \leq 8$ in DIC satisfy the $Q_{g g}$ systematics. The apparent violation of the $Q_{g g}$ systematics at $Z>8$ is due to the influence of the evaporation of nucleons and particles from the excited fragments. To justify the use of the $Q_{g g}$ systematics, statistical arguments, which are not included explicitly in the model, are usually employed. It is therefore not obvious in advance that $P_{Z, N}$, resulting from the master equations, follows the experimentally observed $Q_{g g}$ systematics. Calculations of $P_{Z N}\left(t_{0}=3 \times 10^{-21} \mathrm{~s}, T=1.0\right.$ and $\left.2.0 \mathrm{MeV}\right)$ for the ${ }^{197} \mathrm{Au}+{ }^{40} \mathrm{Ar}(292 \mathrm{MeV}$ ) reaction (Fig. 13) demonstrate that the $Q_{g g}$ systematics with allowance for corrections for nonpairing of the nucleons in their transfer from the donor to the acceptor nucleus is reproduced by the model. The dashed lines in Fig. 13 are fits to the experimental values.

In accordance with the $Q_{g g}$ systematics, the cross-sections for production of isotopes can be expressed as

$\sigma \sim \exp \left[\left(Q_{g g}-\delta+\Delta E_{\mathrm{C}}\right) / T_{0}\right]$,

where $\Delta E_{\mathrm{C}}$ is the change of the Coulomb energy of the system due to proton transfer, and $\delta$ is the correction for the non- 


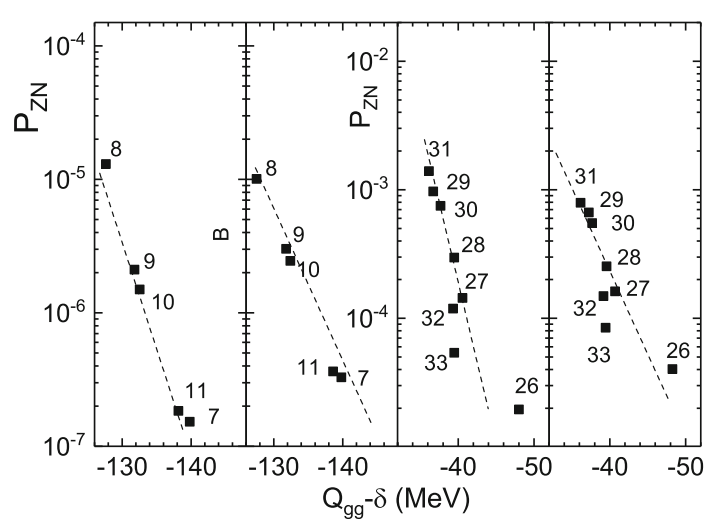

Fig. 13 Production cross-sections of $\mathrm{Al}$ and $\mathrm{Be}$ isotopes as functions of $\left(Q_{g g}-\delta\right)$, calculated for two values of the temperature, $T=1.0$ and $2.0 \mathrm{MeV}\left(t_{0}=3 \times 10^{-21} \mathrm{~s}\right)$ (squares), in the ${ }^{197} \mathrm{Au}+{ }^{40} \mathrm{Ar}(292 \mathrm{MeV})$ reaction [167]. The numbers next to the symbols are the mass numbers of isotopes. The dashed lines correspond to the approximation of the experimental data of Ref. [57]

pairing of the nucleons transferred. The parameter $T_{0}$ differs from the thermodynamic temperature, i.e., the slope of the lines in the $Q_{g g}$ systematics characterizes $T$ only through the functional dependence of $T_{0}$ and $T$. The slope of the lines in Fig. 13 decreases with increasing $T$, i.e., the dependence (46) is qualitatively reproduced. However, it changes by less than a factor 2, confirming the nonequivalence of $T_{0}$ and $T$.

As found, the calculations, which use only the potentialenergy surface, do not always correctly describe the DNS evolution. For example, these models are unable to explain the large variance of the mass distribution and a small change of the mean value $\langle A\rangle$ in the ${ }^{100} \mathrm{Mo}+{ }^{40} \mathrm{Ar}(270 \mathrm{MeV})$ reaction $[89,130]$. The reason is that the DNS evolution is affected not only by the relative positions of the Fermi surfaces of protons and neutrons in the interacting nuclei but also by the details of the shell structure (the number of free and occupied levels near the Fermi surface, the degree of their degeneracy, etc.). The first effect is taken into account by the potential surface. However, the others can be included in the consideration only microscopically. The model, which takes explicitly into account the shell effects for protons and neutrons, well describes the experimental results (Fig. 14).

\subsection{Simplified statistical method}

The production cross-section $\sigma_{Z, N}$ of a primary light nucleus in transfer reactions is the product of the capture cross-section $\sigma_{\text {cap }}$ in the entrance reaction channel and the formation-decay probability $Y_{Z, N}$ of the DNS configuration with charge and mass asymmetries given by $Z$ and $N$ :

$\sigma_{Z, N}=\sigma_{\text {cap }} Y_{Z, N}$.

The primary light neutron-rich nuclei are mainly de-excited by neutron or gamma emissions. We consider only the reac-

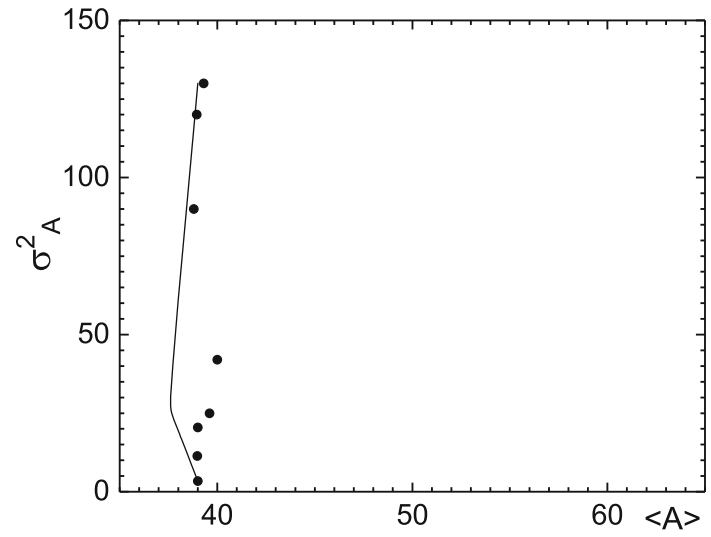

Fig. 14 Dependence of the mass variance $\sigma_{A}^{2}$ on the mean value $\langle A\rangle$ of the light-fragment mass number in the ${ }^{100} \mathrm{Mo}+{ }^{40} \mathrm{Ar}(270 \mathrm{MeV})$ reaction. The bullets show the experimental data of Ref. [130]. The line shows the calculated results [167]

tions leading to the excitation energies of light neutronrich nuclei smaller than their neutron separation energies $S_{n}(Z, N)$. In this case the primary and secondary yields coincide.

If the projectiles and targets are deformed, the value of $E_{\mathrm{c} . \mathrm{m} \text {. }}^{\mathrm{min}}$, at which the collisions of nuclei at all orientations become possible, is larger than the Coulomb barrier calculated for the spherical nuclei. In the collisions with smaller $E_{\text {c.m. }}$ the formation of the DNS is expected to be suppressed. Therefore, we treat $E_{\mathrm{c} . \mathrm{m}} . \geq E_{\mathrm{c} . \mathrm{m}}^{\min }$ for which the capture cross-section is estimated with Eq. (40). Indeed, in the reactions of interest for production of exotic isotopes the values of $E_{\mathrm{c} . \mathrm{m}}$ are always larger than $E_{\mathrm{c} . \mathrm{m} \text {. }}^{\mathrm{min}}$ to have enough energy for the formation of the DNS with very neutron-rich nucleus. The stability of the light neutron-rich nucleus is expected to be smaller in the excited rotational states than in the ground state. In order to produce the exotic nucleus with small angular momentum, only the partial waves with $J \leq 30$ should be considered. Here, we assume that total angular momentum $J$ is distributed in the DNS proportionally to the corresponding moments of inertia and set $J_{\text {cap }}=30$ in Eq. (40).

Based on the idea of $Q_{g g}$ systematics [57], instead of Eq. (34) one can use the simple statistical method to calculate $Y_{Z N}$. This method for finding $Y_{Z, N}$ uses the DNS potential energy calculated as in [167]

$U(R, Z, N, J)=B_{\mathrm{L}}+B_{\mathrm{H}}+V(R, Z, N, J)$,

where $B_{\mathrm{L}}$ and $B_{\mathrm{H}}$ are the mass excesses of the light and heavy fragments, respectively, which are taken from Ref. [206] for known nuclei and from Ref. [207] for unknown nuclei.

One can conclude from the calculations with Eq. (30) that the quasi-stationary regime is established quite fast in the DNS considered, specially along the trajectory in charge (mass) asymmetry corresponding to $N / Z$ equilibrium in the 
DNS. For this trajectory, $N=N_{0}(Z)$, i.e. the neutron number follows $Z$. Therefore, the formation probability for the configuration with $Z$ and $N_{0}(Z)$ is estimated as

$$
\begin{aligned}
& P_{Z, N_{0}}\left(t_{0}\right) \approx C \\
& \quad \times \exp \left(-\frac{U\left(R_{m}, Z, N_{0}, J\right)-U\left(R_{m}, Z_{i}, N_{i}, J\right)}{\Theta\left(Z_{i}, N_{i}\right)}\right),
\end{aligned}
$$

where $C$ is a normalized constant. The temperature $\Theta\left(Z_{i}, N_{i}\right)$ is calculated by using the Fermi-gas expression $\Theta\left(Z_{i}, N_{i}\right)=$ $\sqrt{E^{*} / a}$ with the excitation energy $E^{*}\left(Z_{i}, N_{i}\right)$ of the initial DNS and with the level-density parameter $a=A_{\text {tot }} / 12$ $\mathrm{MeV}^{-1}$, where $A_{\text {tot }}$ is the total mass number of the system.

The formation of the DNS containing the light neutronrich nucleus with given $Z$ is considered as a two-step process. The formation of the DNS with $Z$ and $N_{0}$ is firstly treated. Then one should calculate the probability $G_{Z, N}=$ $\Lambda_{Z, N, N_{0}}^{R} t_{0}$ of the formation and decay of the DNS with exotic nucleus. Since the DNS with $Z$ and $N_{0}$ is in the conditional minimum of the potential-energy surface, we use the Kramers-type expressions for the quasi-stationary rate $\Lambda_{Z, N, N_{0}}^{R}$ of decay through the barrier $B_{R}(Z, N)=$ $U\left(R_{b}, Z, N, J\right)-U\left(R_{\mathrm{m}}, Z, N_{0}, J\right)$ which this DNS should overcome to observe the decay of the DNS with $Z$ and $N$ :

$$
\Lambda_{Z, N, N_{0}}^{R}=\kappa_{R}\left(Z, N, N_{0}\right) \exp \left(-\frac{B_{R}(Z, N)}{\Theta\left(Z, N_{0}\right)}\right),
$$

where the preexponential factor depends on the friction and stiffness of the potential at the minimum and on the barrier. The temperature $\Theta\left(Z, N_{0}\right)$ is calculated for the excitation energy $E^{*}\left(Z_{i}, N_{i}\right)-\left[U\left(R_{\mathrm{m}}, Z, N_{0}, J\right)-\right.$ $\left.U\left(R_{\mathrm{m}}, Z_{i}, N_{i}, J\right)\right]$.

The main factor which restricts the time $t_{0}$ of the reaction and prohibits the formation of DNS containing the exotic nuclei is the evolution of the initial DNS to more symmetric configurations and decay of the DNS during this process. Therefore, the time of decay in $R$ from the initial configuration or from more symmetric configurations mainly determines $t_{0}$. We use again the Kramers-type expression for the quasi-stationary rate $\Lambda_{Z_{i}, N_{i}}^{R}$ of decay through the barrier $B_{R}\left(Z_{i}, N_{i}\right)=B_{\mathrm{qf}}\left(Z_{i}, N_{i}\right)=U\left(R_{b}, Z_{i}, N_{i}, J\right)-$ $U\left(R_{\mathrm{m}}, Z_{i}, N_{i}, J\right)$ and the rate $\Lambda_{Z_{i}, N_{i}}^{\eta_{\mathrm{sym}}}$ of symmetrization of the initial DNS through the barrier $B_{\eta_{\text {sym }}}$ in the direction to more symmetric configurations:

$$
\begin{aligned}
& \Lambda_{Z_{i}, N_{i}}^{R}=\kappa_{R}\left(Z_{i}, N_{i}\right) \exp \left(-\frac{B_{\mathrm{qf}}\left(Z_{i}, N_{i}\right)}{\Theta\left(Z_{i}, N_{i}\right)}\right), \\
& \Lambda_{Z_{i}, N_{i}}^{\eta_{\mathrm{sym}}}=\kappa_{\eta_{\mathrm{sym}}}\left(Z_{i}, N_{i}\right) \exp \left(-\frac{B_{\eta_{\mathrm{sym}}}\left(Z_{i}, N_{i}\right)}{\Theta\left(Z_{i}, N_{i}\right)}\right) .
\end{aligned}
$$

Therefore, $t_{0}=1 /\left(\Lambda_{Z_{i}, N_{i}}^{R}+\Lambda_{Z_{i}, N_{i}}^{\eta_{\text {sm }}}\right)$. Since $B_{\eta_{\text {sym }}}\left(Z_{i}, N_{i}\right)=$ 0.5-1.5 MeV and $B_{\mathrm{qf}}\left(Z_{i}, N_{i}\right) \geq 4 \mathrm{MeV}$ in the considered reactions, $\Lambda_{Z_{i}, N_{i}}^{R} \ll \Lambda_{Z_{i}, N_{i}}^{\eta_{\text {sym }}}$ and $t_{0} \approx 1 / \Lambda_{Z_{i}, N_{i}}^{\eta_{\text {sym }}}$. Therefore, we can calculate $Y_{Z, N}$ as

$Y_{Z, N}=P_{Z, N} \Lambda_{Z, N, N_{0}}^{R} t_{0}$.

This expression leads to $Y_{Z_{i}, N_{i}} \approx 0.05$ that is consistent with our previous calculations [20]. In the calculation of $Y_{Z, N}$ the uncertainty related to the preexponential factor is estimated within the factor of 1.5 .

The suggested simplified approach is suitable if the initial DNS point in the reaction is located close to the $N / Z$ equilibrium. If the injection point is considerably displaced from the $N / Z$ equilibrium, the dynamical effects mainly contribute to the production of nuclei near the injection point and our statistical approach underestimates their yields.

The exotic nucleus as well as any nucleus far from the entrance channel of the reaction are the result of MNT between the projectile-like and target-like parts of the DNS. As a result, we can assume the thermal equilibrium in the DNS containing the exotic nucleus or in the DNS which is quite far from the initial DNS in the space $(N, Z)$. Indeed, for the formation of these DNS one needs quite a long time $t_{0} \approx 10^{-20} \mathrm{~s}$ at $J \leq 30$. This allows us to assume the same temperature in the DNS nuclei and to define the excitation energy of the light nucleus with the mass $A_{L}$ as $E_{L}^{*}(Z, N)=$ $\left[E^{*}\left(Z_{i}, N_{i}\right)-\left[U\left(R_{\mathrm{m}}, Z, N_{0}, J\right)-U\left(R_{\mathrm{m}}, Z_{i}, N_{i}, J\right)\right]-\right.$ $\left.B_{R}(Z, N)\right] A_{L} / A_{\text {tot }}$. The deviation from the thermal equilibrium is expected only for the DNS decays near the injection point where the temperature of the heavy nucleus is smaller than the temperature of the light nucleus. Thus, assuming the thermal equilibrium in the DNS, we can underestimate the excitation of light primary nucleus and predict the upper limit of $E^{*}\left(Z_{i}, N_{i}\right)$. Note that the partition of excitation energy in the DNS weakly influences $Y_{Z, N}$. Since in our calculations of the DNS potential energy the deformations of the nuclei [208] are close to their values in the ground states, the excitation energies of the DNS nuclei remain almost without changes after the DNS decays.

In order to test our method of calculation of $\sigma_{Z, N}$, we treated the production of Ti in the MNT reactions ${ }^{58} \mathrm{Ni}+{ }^{208} \mathrm{~Pb}$ $\left(E_{\text {c.m. }}=256.8 \mathrm{MeV}\right)$ and ${ }^{64} \mathrm{Ni}+{ }^{238} \mathrm{U}\left(E_{\text {c.m. }}=307.4\right.$ $\mathrm{MeV}$ ) [209-211]. The excitation energies available in these reactions supply 2 neutron evaporation from the primary Ti isotopes having the maximal yields. In the ${ }^{58} \mathrm{Ni}+{ }^{208} \mathrm{~Pb}$ reaction ${ }^{50} \mathrm{Ti}$ and ${ }^{52} \mathrm{Ti}$ are produced with the cross-sections 1 and $0.2 \mathrm{mb}[209,210]$, respectively, which are consistent with our calculated cross-sections 0.6 and $0.35 \mathrm{mb}$, respec- 


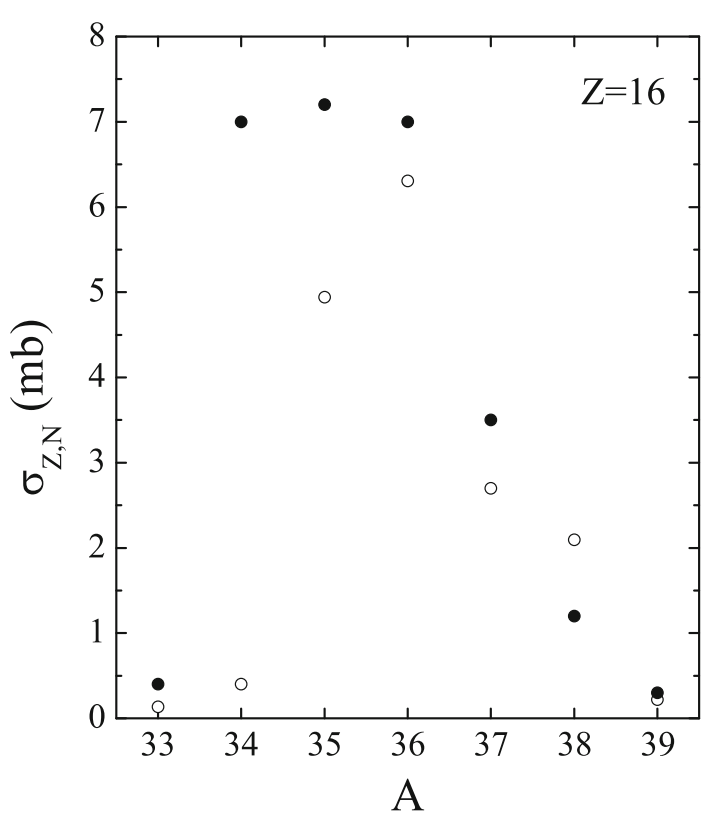

Fig. 15 The calculated (open circles) cross-sections of S isotopes are compared with the experimental ones (solid circles) for the ${ }^{40} \mathrm{Ca}+{ }^{208} \mathrm{~Pb}$ reaction at $E_{\mathrm{c} . \mathrm{m} .}=208.8 \mathrm{MeV}$ [212]

tively. In the ${ }^{64} \mathrm{Ni}+{ }^{238} \mathrm{U}$ reaction the experimental [211] and theoretical cross-sections for ${ }^{52} \mathrm{Ti}$ are 0.5 and $1.6 \mathrm{mb}$, respectively. The description of the yields of $\mathrm{S}$ isotopes in the ${ }^{40} \mathrm{Ca}(249 \mathrm{MeV})+{ }^{208} \mathrm{~Pb}$ reaction [212] is also a good verification of the model. Since the initial DNS in this reaction is not in the $N / Z$-equilibrium, we expect considerable contribution of the non-equilibrium processes to the yields of the products near the entrance channel; the yields of $\mathrm{Ca}$, $\mathrm{K}$, $\mathrm{Ar}$, and $\mathrm{Cl}$ seem to be described better with the dynamical models [167,198-200]. The yields of S isotopes, which are four protons from the entrance channel, are expected to be ruled by the potential-energy surface, and the simplified statistical method therefore seems to be suitable. Aside from the yield of ${ }^{34} \mathrm{~S}$, the calculated yields of $\mathrm{S}$ isotopes are in good agreement with the experimental data in Fig. 15. Therefore, the suggested method is suitable for prediction of the crosssections for the products of MNT reactions.

If the excitation energy of the primary products exceed the thresholds for neutron or charged particle emission, or we are interested in the production of neutron-deficient isotopes, the de-excitation of the primary products is taken into account. According to the model, there are nucleon drift and nucleon diffusion between the DNS nuclei which lead to the formation of excited CN and DNS configurations (DNS with different $Z$ and $A$ ) with probabilities depending on the potential-energy surface and temperature of the system. The decay of excited $\mathrm{CN}$ and DNS configurations is described in a unique way, giving us an opportunity to calculate the production crosssections for residual nuclei in both, light particle and heavy cluster emission channels. Thus, light particle evaporation and complex fragment emissions are treated in the same way and the cluster emission is described under the assumption that the clusters are produced by the collective motion of the nuclear system in the charge asymmetry coordinate, with further thermal escape over the corresponding Coulomb barrier.

The cross-section of the residual nucleus with certain mass number $A$ and charge number $Z$ is given as

$$
\begin{aligned}
\sigma_{Z, A}\left(E_{\text {c.m. }}\right) & =\sum_{J=0}^{J_{\max }} \sigma_{Z, A}\left(E_{\text {c.m. }}, J\right) \\
& =\sum_{J=0}^{J_{\max }} \sigma_{\text {cap }}\left(E_{\text {c.m. }}, J\right) W_{Z, A}^{\prime}\left(E_{\text {c.m. }}, J\right),
\end{aligned}
$$

where $\sigma_{\text {cap }}$ is the partial capture cross-section which defines the transition of the colliding nuclei over the Coulomb barrier and the formation of the initial DNS when the kinetic energy $E_{\text {c.m. }}$ and angular momentum $J$ of the relative motion are transformed into the excitation energy and angular momentum of the DNS. The probability for the production of certain residual nucleus $(Z, A)$ from the excited entrance channel DNS in the distinct decay channel is described by $W_{Z, A}\left(E_{\text {c.m. }}, J\right)$. To calculate $W_{Z, A}^{\prime}\left(E_{\text {c.m. }}, J\right)$, one has to find the formation-emission probability $W_{Z_{1}, A_{1}}\left(E_{\mathrm{c} . \mathrm{m} .}, J\right)$ of a certain light particle or cluster $\left(Z_{1}, A_{1}\right)$ from the excited system. Here, we consider the decay of the excited nuclear system as a sequential light particle $\left(Z_{1}<2\right)$ evaporation, which includes neutrons, protons, deuterons, and tritons, and complex clusters $\left(Z_{1} \geq 2\right)$. The maximum value of the angular momentum $J_{\max }$ is limited either by the kinematic angular momentum $J_{\max }^{\mathrm{kin}}$ or by the critical angular momentum $J_{\mathrm{cr}}$ depending on which one is smaller: $J_{\max }=\min \left\{J_{\max }^{\mathrm{kin}}, J_{\mathrm{cr}}\right\}$.

The emission probability $W_{Z_{1}, A_{1}}\left(E_{\text {c.m. }}, J\right)$ of a light particle or cluster $\left(Z_{1}, A_{1}\right)$ is calculated as the product of the $\mathrm{CN}$ or DNS formation probability and the $\mathrm{CN}$ or DNS decay probability:

$W_{Z_{1}, A_{1}}\left(E_{\text {c.m. }}, J\right)=\frac{P_{Z_{1}, A_{1}} P_{Z_{1}, A_{1}}^{R}}{\sum_{Z_{1}^{\prime}, A_{1}^{\prime}} P_{Z_{1}^{\prime}, A_{1}^{\prime}} P_{Z_{1}^{\prime}, A_{1}^{\prime}}^{R}}$,

where the indices $Z_{1}^{\prime}$ and $A_{1}^{\prime}$ go over all possible channels from the neutron evaporation to the symmetric splitting. In the equilibrium limit of the master equation in the charge and mass asymmetries (see [213,214] for details) the probability $P_{Z_{1}, A_{1}}$ is given by

$P_{Z_{1}, A_{1}}\left(E_{\text {c.m. }}, J\right) \sim \exp \left[-U\left(R_{m}, Z_{1}, A_{1}, J\right) / T_{\max }(J)\right]$.

Here, $n, p, d$, and $t$-evaporation channels are taken into consideration with $U\left(R_{\mathrm{m}}, Z_{1}, A_{1}, J\right)=0$. The quasi-fission barrier $B_{R}^{\mathrm{qf}}$, calculated as the difference between the bottom of the inner pocket and the top of external barrier, prevents the decay of the DNS in $R$ with the factor $P_{Z_{1}, A_{1}}^{R}$ given as 
$P_{Z_{1}, A_{1}}^{R}\left(E_{\text {c.m. }}, J\right) \sim \exp \left[-B_{R}^{\mathrm{qf}}\left(Z_{1}, A_{1}, J\right) / T_{Z_{1}, A_{1}}(J)\right]$.

In Eqs. (55) and (56), $T_{\max }(J)=\max \left\{T_{Z_{1}, A_{1}}(J)\right\}$, where $T_{Z_{1}, A_{1}}(J)$ are the temperatures of the $\mathrm{CN}$ and all possible DNS. For the emission of particles with $Z_{1}<2, T_{Z_{1}, A_{1}}(J)=$ $T_{C N}(J)$ is the temperature of the $\mathrm{CN}$ and $B_{R}^{\mathrm{qf}}\left(Z_{1}, A_{1}, J\right)$ is equal to the particle binding energy plus the value of the corresponding Coulomb barrier at $Z_{1} \neq 0$. The Fermi-gas model is employed to compute the temperature.

The neutron-deficient isotopes of nuclei are usually produced in very weak decay channels and their absolute production cross-sections are very sensitive to the excitation energy which is available for light particle or cluster emission. After emission of light particles or clusters, the excitation energy of the residual nucleus depends on emission barrier $B_{R}^{\mathrm{qf}}\left(Z_{1}, A_{1}, J\right)$ and kinetic energy of the emitted particle or cluster. In Refs. [213,214], the kinetic energies of emitted particles have been assumed to be $2 T_{Z, A}(J)$ in the average. Here, we modified our calculations by taking into account the kinetic energy distributions of emitted light particles $(n$, $p, d$, and $t)$ and clusters $\left({ }^{3} \mathrm{He},{ }^{4} \mathrm{He},{ }^{12,14} \mathrm{C}, \ldots\right)$ as

$P(\epsilon, J)=\epsilon \exp \left[-\epsilon / T_{Z, A}(J)\right]$,

where $\epsilon$ is the kinetic energy of the light particle or cluster in the center-of-mass system. The actual value of $\epsilon$ in each decay event is chosen by the Monte Carlo method. This modification affects the cross-sections in very weak decay channels and has almost no influence on the major decay channels.

In the calculations, Eqs. (53) and (54) are used to treat the sequential statistical decay (the evaporation of light particles and/or the binary decay) of the hot $\mathrm{CN}$ until all fragments become cold (the excitation energy of fragments is smaller than its neutron emission threshold). The number $n$ of the generated events in the Monte Carlo method was chosen according to the smallest decay probability, which is $\sim 1 / n$. Number $n>10^{4}$ of iterations is large enough for obtaining the calculated results with quite a high accuracy.

To test the method suggested, we treated the production cross-sections of exotic nuclei in very weak decay channels. In Fig. 16, the calculated cross-sections are in a good agreement with the experimental data for the ${ }^{58} \mathrm{Ni}+{ }^{54} \mathrm{Fe}$ reaction [215]. Note that our model reproduces the experimental data without adjusting the separation energies of light particles and clusters. So, the model can be employed for the predictions of excitation functions of exotic nuclei in the completefusion and QF reactions.

The results of the calculations [216] show that the maximum production cross-sections of ${ }^{100} \mathrm{Sn}$ are about $30 \mathrm{nb}$ in the ${ }^{58} \mathrm{Ni}+{ }^{46} \mathrm{Ti}$ reaction with stable beam and about $1 \mu \mathrm{b}$ in the reactions with radioactive beams (Fig. 17). By taking into
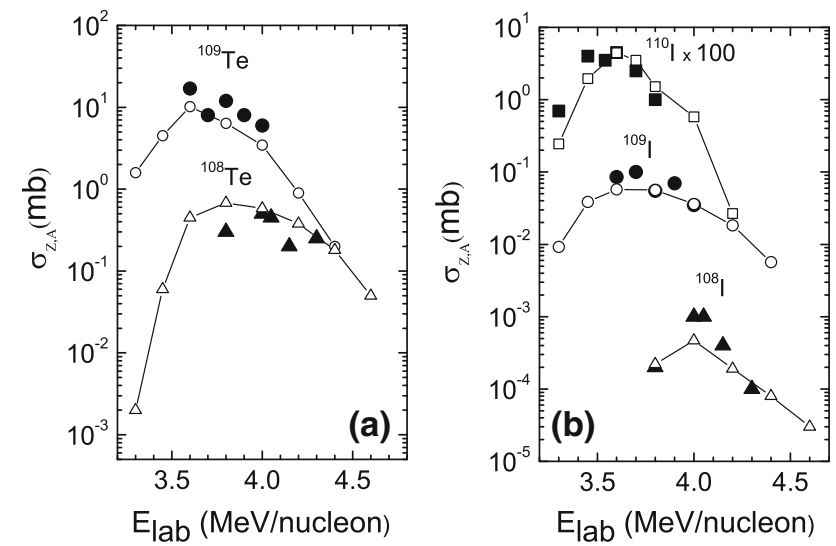

Fig. 16 Comparison of experimental [215] (solid symbols) and calculated [216] (open symbols connected by line) excitation functions for production of the indicated isotopes of $\mathrm{Te}(\mathbf{a})$ and $\mathrm{I}(\mathbf{b})$ in the ${ }^{58} \mathrm{Ni}+{ }^{54} \mathrm{Fe}$ reaction
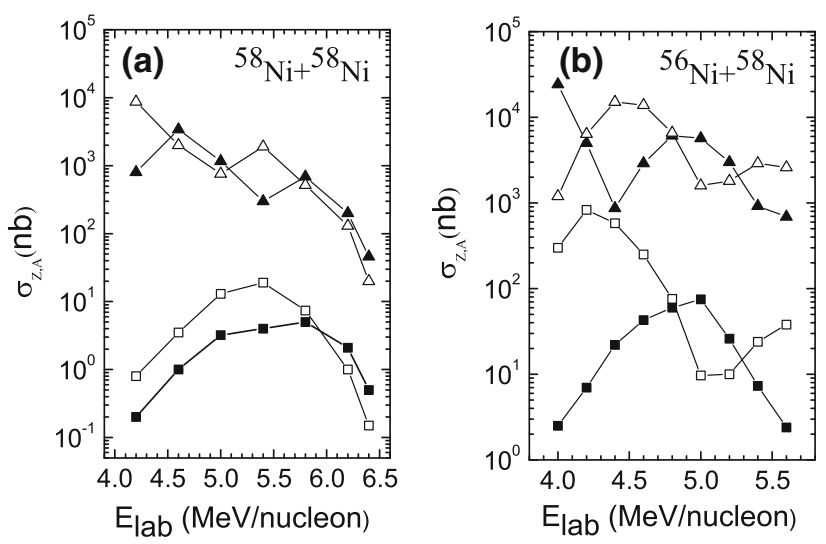

Fig. 17 The predicted [216] excitation functions for production of ${ }^{100} \mathrm{Sn}(\square),{ }^{101} \mathrm{Sn}(\square),{ }^{102} \mathrm{Sn}(\Delta)$, and ${ }^{103} \mathrm{Sn}(\triangle)$ in $x n$-decay channels of the reactions ${ }^{58} \mathrm{Ni}+{ }^{46} \mathrm{Ti}(\mathbf{a})$ and ${ }^{56} \mathrm{Ni}+{ }^{46} \mathrm{Ti}(\mathbf{b})$

consideration the beam intensities and optimal production cross-sections, we found that the best candidate for the production of ${ }^{100} \mathrm{Sn}$ among reactions with radioactive ion beams is the ${ }^{56} \mathrm{Ni}+{ }^{46}$ Ti reaction via $2 n$ emission channel. The production cross-sections of the doubly magic nucleus ${ }^{100} \mathrm{Sn}$ via light particle evaporation and cluster emission channels are comparable. The production cross-sections for the nuclei ${ }^{101-103}$ Sn via $x n, p x n, \alpha x n$ and cluster decay channels are predicted as well in Ref. [216].

\section{Nucleosynthesis in transfer reactions}

\subsection{Early discoveries of new isotopes in MNT reactions}

Shortly after their discovery, MNT reactions revealed already their potential for synthesis of new isotopes. About 75 new nuclides were discovered in the years 1969-1995 in different laboratories. All of them are on the neutron-rich side 


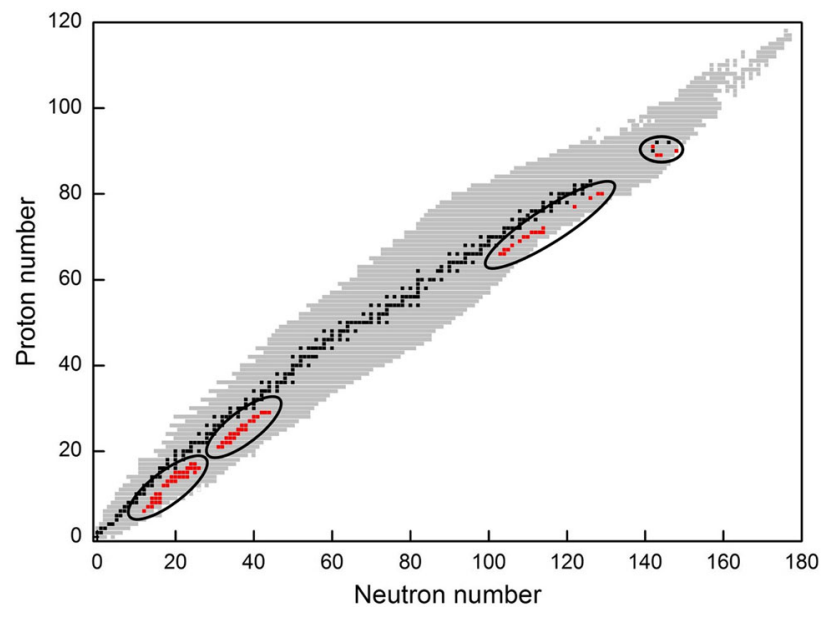

Fig. 18 Chart of nuclides with all presently known isotopes. The 76 nuclides which were discovered in MNT reactions are marked in red. They are all located on the neutron-rich side

of the chart of nuclides (Fig. 18). First, Dubna published in the years 1969-1971 the observation of nearly 30 new projectile-like nuclides of elements from carbon $(Z=6)$ to chlorine $(Z=17)[8,10,217,218]$ in MNT reactions of $\mathrm{O}, \mathrm{Ne}$ and $\mathrm{Ar}$ ions with ${ }^{232} \mathrm{Th}$ targets at beam energies of about $50 \%$ above the Coulomb barrier. The reaction products were separated according to their magnetic rigidity $B \rho$ using a magnetic analyzer which was typically placed at angles around $50^{\circ}$ with respect to the beam axis. In the focal plane of the separator, the energy loss $\Delta E$ and total energy $E$ of the ions was measured with an $E-\Delta E$ silicon detector telescope to determine the proton number $\mathrm{Z}$ of the ions. Ten years after the first discoveries in Dubna, scientists at Lawrence Berkeley National Laboratory (LBNL) in California and at Orsay (France) observed a further series of new isotopes with atomic numbers $Z=20$ to $Z=30$ in MNT reactions of Ar and Fe beams with ${ }^{238} \mathrm{U}$ targets [219-221]. Both laboratories applied TOF- $E-\Delta E$ telescopes to obtain the atomic number $Z$ and mass number $A$ of the nuclei.

Around 1980 also GSI Darmstadt entered the stage of MNT reactions and in the following 12 years about 15 new isotopes in the rare earth region were synthesized [222-229]. Typical for these experiments was the application of relatively heavy and symmetric collision systems like $\mathrm{Xe}+\mathrm{Ta}$ or $\mathrm{W}+\mathrm{W}$. The reaction products were separated according to $B \rho$ with the GSI Online Mass Separator [230] and then stopped and accumulated in a catcher foil inside an ion source. From there, they were extracted as singly charged ions, re-accelerated to $60 \mathrm{keV}$ and then implanted again. Using these samples with implanted reaction products, the isotopes were identified offline in $\beta-, \gamma-$, and X-ray decay spectroscopy. With this setup, also the then heaviest new MNT products ${ }^{205} \mathrm{Au}$ [231] and ${ }^{232,234} \mathrm{Ac}$ [232] were discovered, produced in collisions of ${ }^{208} \mathrm{~Pb}$ and ${ }^{238} \mathrm{U}$ beams with

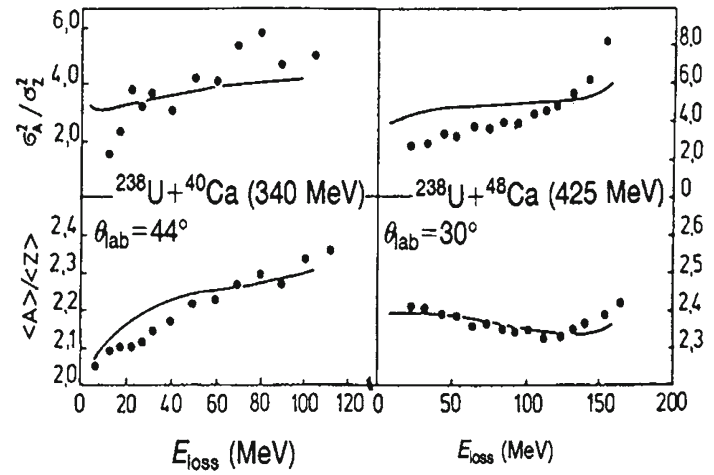

Fig. 19 Dependence of $\langle A\rangle /\langle Z\rangle$ (lower part) and $\sigma_{A}^{2} / \sigma_{Z}^{2}$ (upper part) on the kinetic energy loss in the ${ }^{40,48} \mathrm{Ca}+{ }^{238} \mathrm{U}$ reactions. The dots are the experimental data of Refs. $[67,68]$. The calculated results [205] are shown by lines

$\mathrm{W}$ and Ta targets. The $B \rho-\Delta E-E$ and TOF- $-\Delta E-E$ methods lead to mass (charge) resolutions of about $1 \%$. Therefore it is only applicable for lighter nuclei, while the heavy MNT products were identified by decay tagging. This situation is still the same today.

To get an idea about the $N / Z$-equilibrium in DIC, we show in Fig. 19 exemplarily the results for ${ }^{40} \mathrm{Ca}+{ }^{238} \mathrm{U}(340$ $\mathrm{MeV})$ and ${ }^{48} \mathrm{Ca}+{ }^{238} \mathrm{U}(425 \mathrm{MeV})$ collisions. The experimental data $[67,68]$ are compared with DNS model calculations [205]. The calculations were performed for the angular momenta close to the critical value $J_{\text {crit }}$ being characteristic of DIC. Good agreement between theoretical and experimental results is achieved when one considers the dependencies of the ratios $\sigma_{A}^{2} / \sigma_{Z}^{2}$ and $\langle A\rangle /\langle Z\rangle$ on energy loss $E_{\text {loss }}$ (Fig. 19). At small energy losses, $\sigma_{A}^{2} / \sigma_{Z}^{2}$ is close to $\langle A\rangle /\langle Z\rangle$, and, as is well known [64], this means that proton exchange is dominant in the initial stage of the reaction. With increasing interaction time (increase of $E_{\text {loss }}$ ), the ratio $\sigma_{A}^{2} / \sigma_{Z}^{2}$ becomes close to $(\langle A\rangle /\langle Z\rangle)^{2}$, which corresponds to a strong correlation between the proton and neutron transfers. Analyzing the dependencies in Fig. 19, we may conclude that the establishment of $N / Z$ equilibrium is a continuous process.

\subsection{Search for superheavy nuclei in MNT reactions}

\subsubsection{Early experiments}

In parallel to the above described experiments, MNT reactions were explored already in their early days with the goal to synthesize (new) superheavy nuclei. Typical for these experiments was the application of heavy actinide targets from uranium $(Z=92)$ to einsteinium $(Z=99)$ in combination with various projectiles from $\mathrm{O}$ to $\mathrm{U}$ at energies up to $\sim 20 \%$ above the Coulomb barrier. Pioneering results were obtained by a collaboration of nuclear chemists in experiments at LBNL and at GSI, where they observed numer- 
ous MNT products with atomic numbers $Z=(94-103)$ [86,233-238]. Their results demonstrated that MNT reactions are suitable to populate also nuclei beyond uranium, although no new isotopes were observed then. The isotope identification was performed with radiochemical methods which one can roughly distinguish in on-line and off-line experiments. In on-line experiments, the nuclei were produced in a He gas filled target chamber and transported by gas-jet technique to chemical separation devices to separate the different chemical elements. After separation, samples were prepared for identification of the isotopes by decay tagging. The minimum time for one such cycle was about $60 \mathrm{~s}$, which determines the minimum required half-lives of accessible nuclei. For long-living reaction products the easier off-line technique was used, where the reaction products were implanted in a catcher foil behind the target, followed by chemical element separation and decay tagging for isotope identification. The minimum time for a full cycle was about $30 \mathrm{~min}$. The radiochemical methods were the most sensitive ones of these first generation experiments, leading to lower limit cross-sections of $20 \mathrm{nb}$. Figure 20 gives an overview on the most heavy transfer products observed in these experiments with ${ }^{16,18} \mathrm{O},{ }^{20,22} \mathrm{Ne},{ }^{40,44,48} \mathrm{Ca}$, and ${ }^{238} \mathrm{U}$ projectiles in combination with ${ }^{238} \mathrm{U},{ }^{248} \mathrm{Cm}$, and ${ }^{254} \mathrm{Es}$ targets [86,233-239]. In all reactions, quite neutron-rich nuclei were populated.

\subsubsection{New model predictions}

After the mid 1990s, the interest in MNT reactions faded, but already a decade later their comeback started. It was triggered by the quest for appropriate methods to synthesize new neutron-rich superheavy nuclei, which cannot be reached in complete-fusion reactions with stable projectile and target nuclei. The DNS model [22] and the Langevin model $[33,51,52]$ predicted cross-sections which gave cause for optimism and stimulated new experimental activities. However, these models result in two principally different points of view concerning the optimal projectile-target combinations. The DNS model, which uses diabatic internuclear potentials, suggests to use intermediate heavy neutron-rich projectiles, preferentially the neutron-rich ${ }^{48} \mathrm{Ca}$, combined with the heaviest available actinide targets like $\mathrm{Pu}, \mathrm{Cm}$ or Cf. It is obvious that such systems can principally not lead to neutron-rich isotopes of the heaviest known elements, but they provide sufficient neutrons to synthesize nuclei below $Z=110$. Figure 21 shows the cross-sections, which are predicted by the DNS model for neutron-rich isotopes of Md to Hs $(Z=101-108)$ in MNT reactions of ${ }^{48} \mathrm{Ca}$ projectiles on ${ }^{244,246,248} \mathrm{Cm}$ targets [22]. Note that in these MNT reactions one can also produce new neutron-rich projectile-like fragments such as ${ }^{84,86} \mathrm{Zn}$ and ${ }^{90,92} \mathrm{Ge}$ with neutron numbers

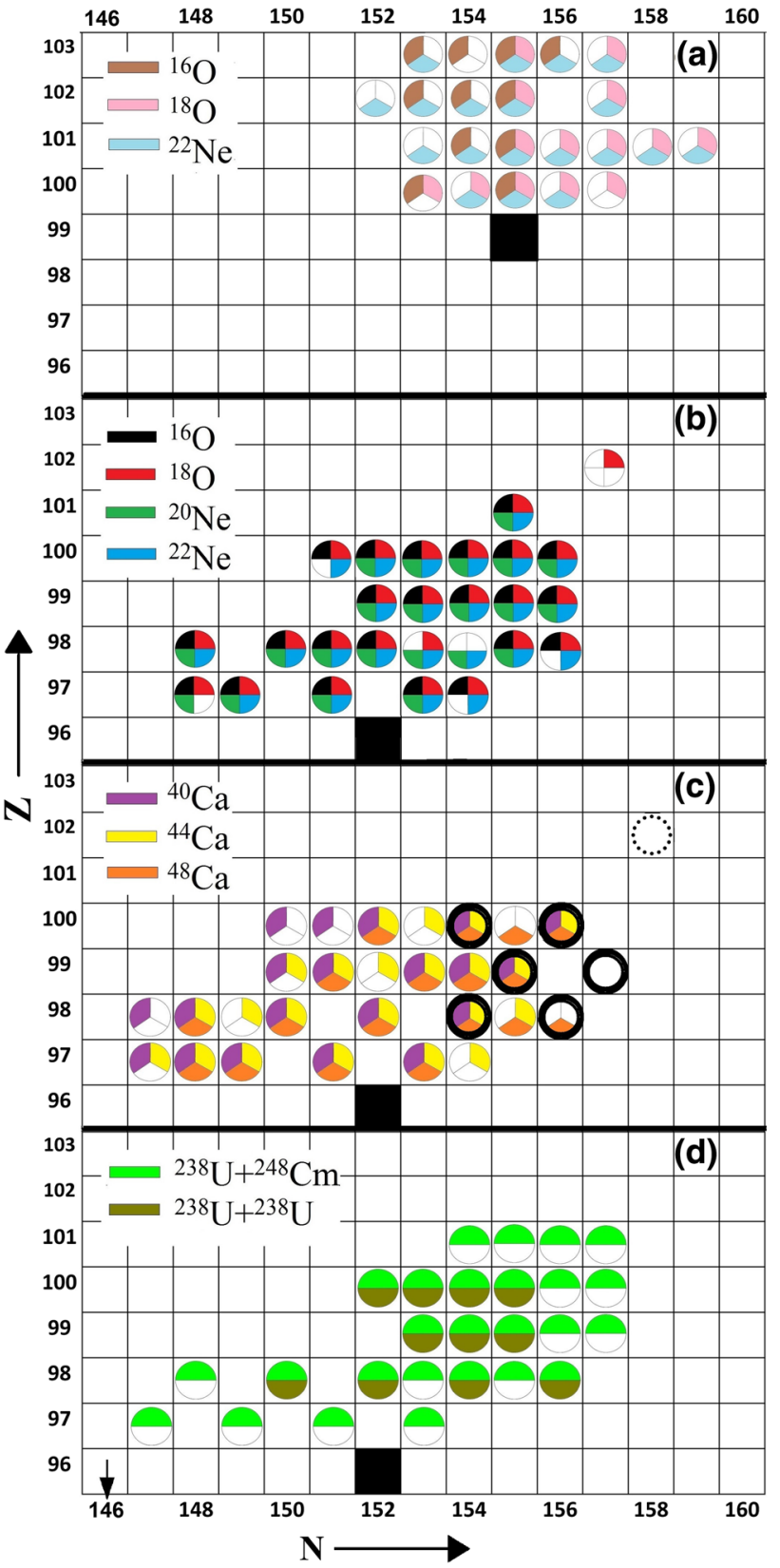

Fig. 20 The so far heaviest observed multinucleon transfer products in reactions of $\mathbf{a}^{16,18} \mathrm{O}$ and ${ }^{22} \mathrm{Ne}$ beams on ${ }^{254}$ Es targets [235], $\mathbf{b}^{16,18} \mathrm{O}$ and ${ }^{20,22} \mathrm{Ne}$ on ${ }^{248} \mathrm{Cm}$ [237], $\mathbf{c}^{40,44,48} \mathrm{Ca}$ on ${ }^{248} \mathrm{Cm}$ [86,238] and $\mathbf{d}^{238} \mathrm{U}$ beams on ${ }^{238} \mathrm{U}$ and ${ }^{248} \mathrm{Cm}$ [234]. The locations of the respective target nuclei are marked by black squares. The nuclei marked with circles in c were observed in our own recent studies of the system ${ }^{48} \mathrm{Ca}$ on ${ }^{248} \mathrm{Cm}$ [239] at the velocity filter SHIP and the arrow in $\mathbf{d}$ indicates the neutron number of the ${ }^{238} \mathrm{U}$ target

beyond $N=50$ [29] or study the transfer-induced fission of isotopes from Md to Hs [240].

The Langevin model, in contrast, suggests to use the heaviest available collision systems like ${ }^{238} \mathrm{U}+{ }^{238} \mathrm{U},{ }^{248} \mathrm{Cm},{ }^{254} \mathrm{Es}$ $[33,51,52]$. Different than in the DNS model, the adiabatic potentials of the Langevin model result also in such giant 


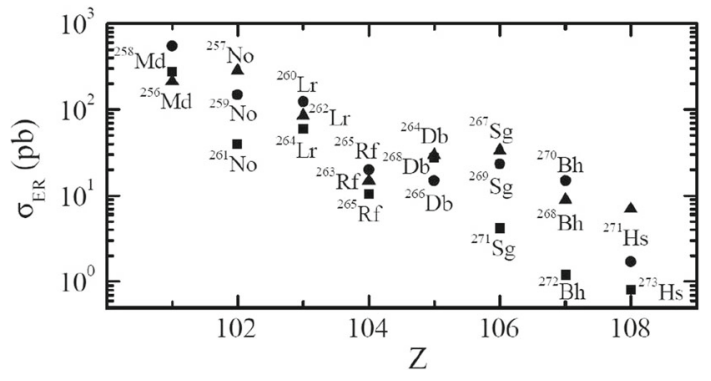

Fig. 21 DNS model calculations of cross-sections for neutron-rich nuclei with $Z=(101-108)$ produced in MNT reactions of ${ }^{48} \mathrm{Ca}$ projectiles on targets of ${ }^{244} \mathrm{Cm}\left(E_{\text {c.m. }} .=207 \mathrm{MeV}\right.$, triangles $),{ }^{246} \mathrm{Cm}$ $\left(E_{\text {c.m. }}=205.5 \mathrm{MeV}\right.$, circles $)$ and ${ }^{248} \mathrm{Cm}\left(E_{\text {c.m. }}=204 \mathrm{MeV}\right.$, squares $)$ [22]. The beam energies were close to the respective Coulomb barrier. The denoted reaction products result after evaporation of one neutron from the excited primary transfer products

systems in a sufficient contribution of collisions with long interaction times up to $5 \times 10^{-21} \mathrm{~s}$ [33], which is a necessary requirement to exchange large amounts of nucleons. The advantage of such heavy systems is that they provide large numbers of neutrons and can principally lead to neutron-rich nuclei of the heaviest known elements. But the cross-sections drop by about one order of magnitude with the transfer of every additional proton like revealed by the calculations in Fig. 22 [52-54]. According to Fig. 22, in the ${ }^{238} \mathrm{U}+{ }^{248} \mathrm{Cm}$ reaction the cross-sections of new neutron-rich Fm isotopes are $\leq 50 \mathrm{nb}$ and for new isotopes of No and Sg they reach values of $\leq 2 \mathrm{nb}$ and $\leq 0.1 \mathrm{pb}$, respectively.

Finally, from the different model predictions no clear trend becomes apparent if giant collision systems, such as $\mathrm{U}+\mathrm{Cm}$, or systems with lighter projectiles like $\mathrm{Ca}+\mathrm{Cm}$ lead to larger cross-sections. The DNS model cross-sections for nuclei around $Z=100$ are smaller than the respective Langevin model cross-sections. But their decrease with increasing proton number is less steep in the DNS model, resulting finally in larger cross-sections for isotopes in the $\mathrm{Sg}$ region.

Also the TDHF theory was adapted to calculate the MNT cross-sections for production of heavy nuclei $[30,32,39,43$, $44,49,164,241]$. The inverse QF mechanism, which is similar to that in Ref. [22], was proposed to produce neutron-rich transfermium nuclei [30]. The estimated interaction time is close to that obtained with the DNS model. The production of neutron-rich transfermium nuclei in the reactions ${ }^{40,48} \mathrm{Ca}$, ${ }^{58} \mathrm{Ni}+{ }^{238} \mathrm{U},{ }^{248} \mathrm{Cm}$ was also studied within the model [46] based on the DNS concept.

Extensive studies with ${ }^{248} \mathrm{Cm}$ targets were performed in the early experiments and also in a recent experiment by us [239]. They reveal the on first glance striking observation that nearly the same region of isotopes is populated with such different projectiles like $\mathrm{O}, \mathrm{Ne}, \mathrm{Ca}$ or $\mathrm{U}$ and that the cross-sections and positions of the isotopic distributions differ only little (Fig. 23). But this might be because the identi- fied nuclides are still located in the closer target environment and do therefore not yet reveal the diverging of the different collision systems. But despite still existing uncertainties, the different theoretical approaches and available experimental data leave a common picture. The calculated cross-sections for new neutron-rich superheavy MNT products are on the nanobarn scale or below. If we assume that cross-sections in the range $1 \mathrm{pb}$ to $1 \mathrm{nb}$ are accessible, MNT reactions can be an option to reach new isotopes with $Z \leq 108$. For example, the so far not directly produced endpoint nuclei of the decay chains of superheavy isotopes from hot fusion reactions can be reached. The choice of the collision system seems not to be a very critical factor if the envisaged isotopes are not located too far from the entrance channel nuclei.

\subsubsection{New experimental approaches and results}

The theoretical and experimental state of the art seems encouraging and challenging at the same time. Encouraging, because the expected MNT cross-sections principally allow one to synthesize a good number of new transuranium isotopes. And challenging, because the cross-sections are at the present limits of experimental feasibility which requires the development of (new) separation and detection techniques as well as higher beam intensities. The present experimental activities are spread around the world, where Argonne, JINR, GANIL, GSI, Legnaro, MSU, RIKEN and others are participating. The development of separation and detection techniques is ongoing and also first experiments were performed.

New advances into the transuranium region were so far made at the velocity filter SHIP at GSI. The application of a velocity filter for separation of heavy MNT products is a new approach. Due to the acceptance angle of $(0 \pm 2)^{\circ}$, only nuclei which are emitted to forward angles can enter the velocity filter. Consequently, some of the detected nuclei originate from central collisions where projectile and target nucleus reach maximum possible overlap and sticking time at the given beam energy. This is the most favorable configuration for the exchange of large numbers of nucleons leading to the creation of isotopes far from the original projectile and target nuclei. Reaction products which are transmitted through SHIP are implanted in a position sensitive silicon strip detector in the focal plane, where they are identified by their $\alpha$ decays. In addition, also gamma decays are recorded with a germanium clover detector which is positioned behind the silicon detector. This enables also the identification of $\beta$ emitters. This method turned out to be very sensitive leading to lower limit cross-sections of about $1 \mathrm{nb}[242,243]$. Further, the fast In-flight separation allowed one to detect also short-living nuclei with half-lives down to $20 \mu \mathrm{s}$, given by the conversion time plus dead time of the data acquisition system. Meanwhile, the study of MNT reactions in forward 

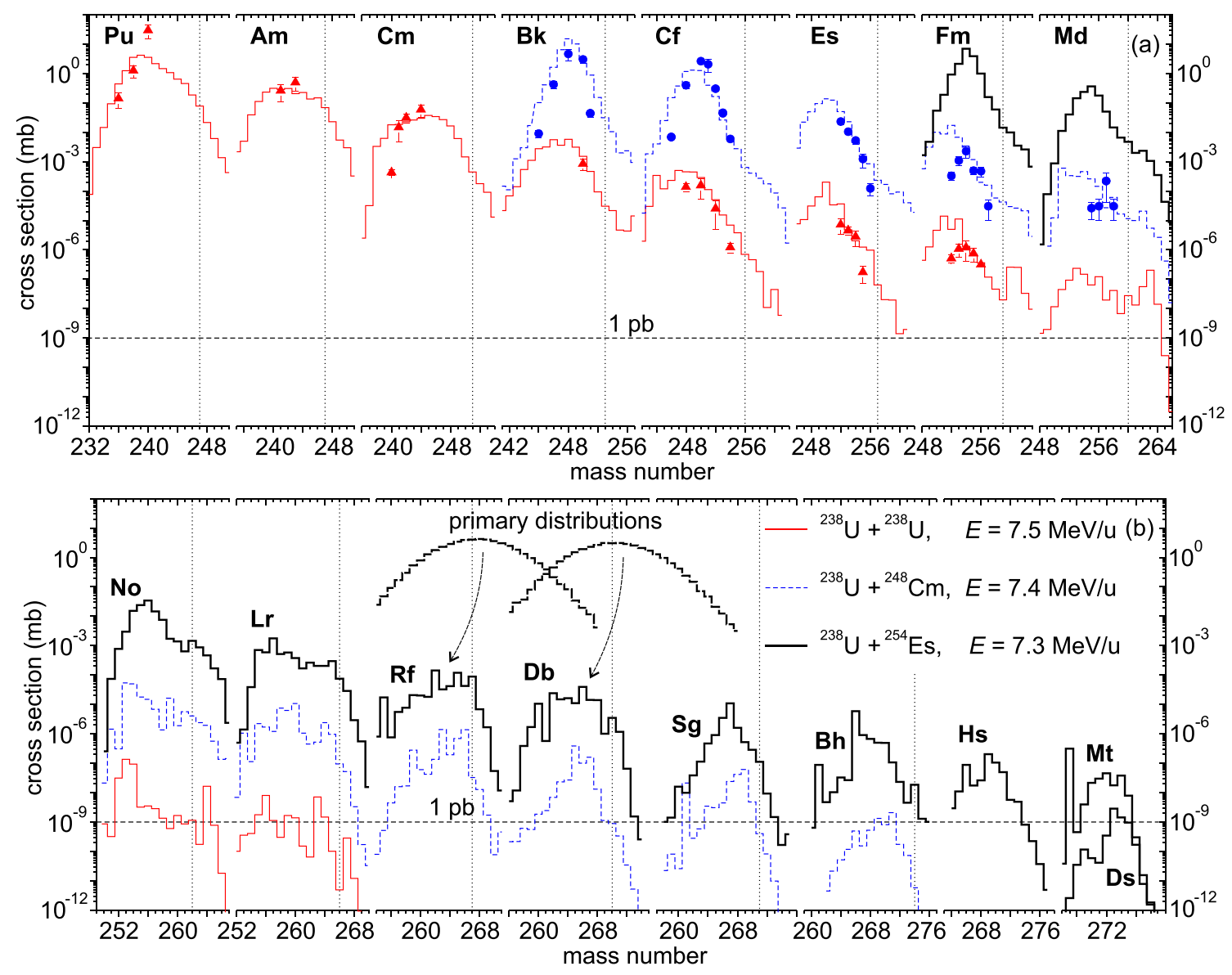

Fig. 22 Isotopic distributions of the above-target products obtained in collisions of actinides. The thin, dashed, and thick histograms correspond to the results of the calculations [52] for the reactions ${ }^{238} \mathrm{U}+{ }^{238} \mathrm{U}(E=7.5 \mathrm{MeV} / \mathrm{u}),{ }^{238} \mathrm{U}+{ }^{248} \mathrm{Cm}(E=7.4 \mathrm{MeV} / \mathrm{u})$, and ${ }^{238} \mathrm{U}+{ }^{254} \mathrm{Es}(E=7.3 \mathrm{MeV} / \mathrm{u})$, respectively. The experimental data for the ${ }^{238} U+{ }^{238} U$ reaction (triangles) are taken from Ref. [233],

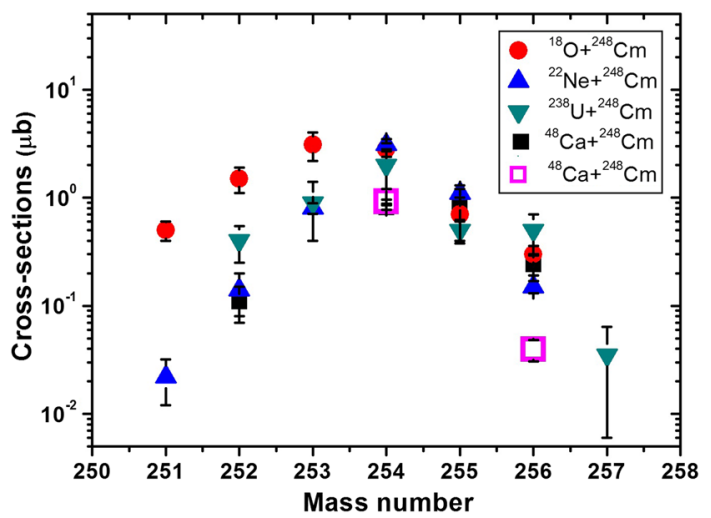

Fig. 23 Cross-sections of $\mathrm{Fm}(Z=100)$ isotopes populated in multinucleon transfer reactions with ${ }^{248} \mathrm{Cm}$ targets (filled symbols). The respective projectiles are indicated in the inset. Fermium is so far the heaviest element for which a broad variety of different isotopes was observed in MNT reactions. The open squares denote the results from our recent experiment with ${ }^{48} \mathrm{Ca}+{ }^{248} \mathrm{Cm}$ at the velocity filter SHIP [239] and for ${ }^{238} \mathrm{U}+{ }^{248} \mathrm{Cm}$ (circles) are from Ref. [234]. For more details, see the text. The heaviest known isotopes of the given chemical elements are indicated by vertical dotted lines. The thick dashed curves show primary (before neutron evaporation) isotopic distributions of $\mathrm{Rf}$ and $\mathrm{Db}$ in the ${ }^{238} \mathrm{U}+{ }^{254}$ Es reaction

direction using In-flight separation, is also tackled by other groups. So far, investigations were also performed at the gasfilled separator TASCA at GSI where heavy MNT products with proton numbers $Z=(83-90)$ were identified in collisions of ${ }^{50} \mathrm{Ti}+{ }^{249} \mathrm{Cf}$ [244]. And measurements at zero degree of light neutron-rich projectile-like nuclei from ${ }^{18} \mathrm{O}+{ }^{238} \mathrm{U}$ reactions were made at GANIL with the LISE achromatic spectrometer [204].

At SHIP, we investigated reactions of ${ }^{48} \mathrm{Ca}+{ }^{248} \mathrm{Cm}$ at an energy slightly above the Coulomb barrier and identified more than 100 different target-like MNT products (Fig. 24). The heaviest ones are two isotopes of Fm and, most likely, the isotope ${ }^{260} \mathrm{No}$, the latter with a total cross-section of $1.7 \mathrm{nb}$, corresponding to three events observed in two days of beamtime. Usually, a contiguous region of isotopes around the target and projectile nucleus is populated in MNT reactions. Therefore, one can assume that also many of the nuclei which are represented by blank squares in Fig. 24 were produced 


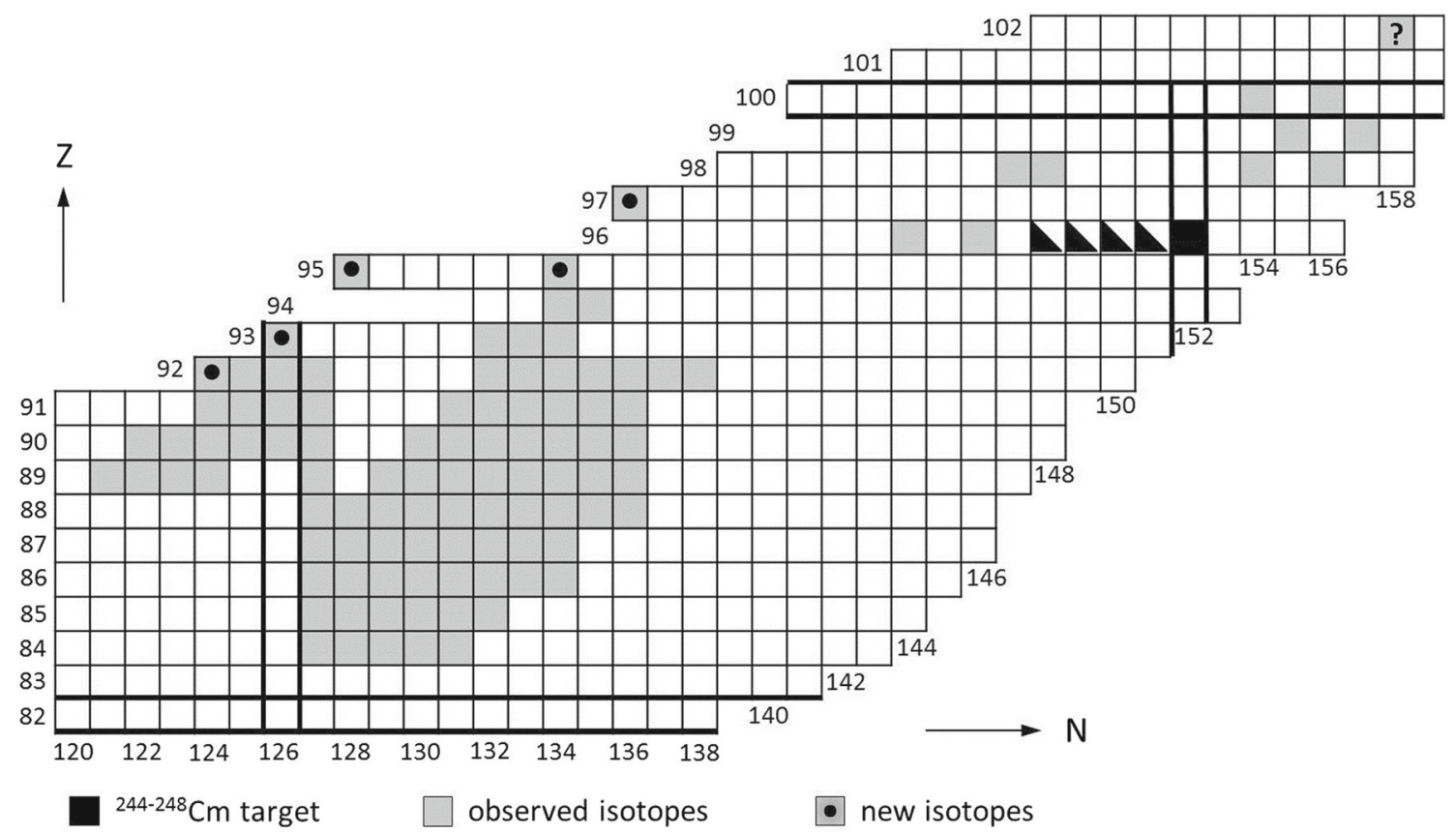

Fig. 24 MNT products (gray squares) observed in reactions of ${ }^{48} \mathrm{Ca}+{ }^{248} \mathrm{Cm}$ at the velocity filter SHIP at GSI [242]. Beside ${ }^{248} \mathrm{Cm}$ with a contribution of $96.85 \%$ (black square), the target contained also the isotopes ${ }^{244-247} \mathrm{Cm}$ with contributions of $0.0007 \%, 0.031 \%, 3.10 \%$ and

but could not be identified by decay tagging due to their too short or long half-lives and/or unfavorable decay channels like fission or beta decay.

Among the observed MNT products were also five new isotopes with $Z \geq 92$. With this, the experiments at SHIP lead for the first time to the discovery of new transuranium isotopes in MNT reactions. Remarkable is that all new isotopes are located on the very neutron-deficient side of the chart. This results from the applied method for isotope identification via $\alpha$ decay tagging, which is so far the only possibility to identify such heavy and slow nuclei. However, the method works only if the nuclei have sufficiently short half-lives and lead to sufficiently long $\alpha$ decay chains. This requirement is mainly fulfilled by neutron-deficient transuranium and superheavy nuclei but largely fails on the neutronrich side of the chart.

Neutron-deficient nuclei around uranium are so far synthesized in fusion-evaporation reactions [245-252]. Therefore, it is interesting to have a closer look and see if MNT reactions might be after all the better alternative to populate the still quite empty neutron-deficient transuranium region. Figure 25 compares measured fusion residue cross-sections of various uranium isotopes [253-259] with the MNT cross-sections measured at SHIP [243]. Figure 25 can be regarded as a representative example because the behavior is very similar for other elements in the uranium region. If cross-sections from
$0.015 \%$ (black triangles). Five new neutron-deficient isotopes were also identified in this experiment and are marked by dots. One of the observed spontaneous fission activities was attributed tentatively to ${ }^{260} \mathrm{No}$, which is indicated by the question mark in the respective square

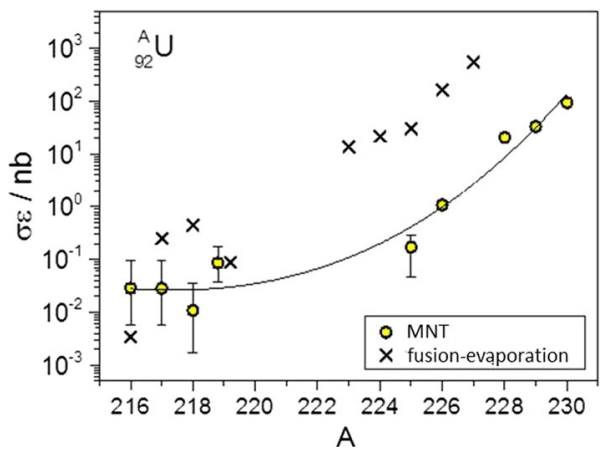

Fig. 25 The product of cross-section and experimental efficiency, $\sigma \varepsilon$, for uranium isotopes measured in fusion-evaporation reactions [253259] (crosses) and in MNT reactions in ${ }^{48} \mathrm{Ca}+{ }^{248} \mathrm{Cm}$ [243] (open circles). For ${ }^{219} \mathrm{U}$ a small offset on the $A$ value is put to avoid an overlap of the data points from complete fusion and MNT

more than one experiment were available for the same isotope, we put the largest known value. Instead of the pure production cross-sections, we put the product of cross-section and experimental efficiency, $\sigma \varepsilon$, because this is the actually relevant parameter which reflects the event count rate (assuming similar beam intensities and target thicknesses). In our example, $\varepsilon$ is mainly determined by the angular acceptance of the applied separators.

Figure 25 indicates that there is no overall valid answer if MNT or complete-fusion reactions are the better option. 
Rather one must distinguish between "neutron-deficient" and "very neutron-deficient" isotopes. For nuclei well above $A=220$ the value of $\sigma \varepsilon$ is significantly larger in fusionevaporation reactions. This is, on one hand, caused by the larger angular acceptances of the separators for fusionevaporation residues. Another reason, on the second hand, is that these isotopes can be produced with very asymmetric projectile-target combinations like ${ }^{22} \mathrm{Ne}+{ }^{208} \mathrm{~Pb} \rightarrow{ }^{230} \mathrm{U}^{*}$ where the low entrance channel Coulomb barriers and favorable $Q$ values result in relatively large fusion residue crosssections. In this mass region, fusion-evaporation reactions appear superior to MNT. The situation changes for very neutron-deficient isotopes where the values of $\sigma \varepsilon$ for MNT and fusion tend to approximate each other, despite the small angular efficiency for MNT products. To reach these nuclei in fusion reactions, more symmetric projectile-target combinations like ${ }^{40} \mathrm{Ar}+{ }^{182} \mathrm{~W} \rightarrow{ }^{222} \mathrm{U}^{*}$ must be applied. The larger entrance channel Coulomb barriers and less favorable $Q$ values lead to relatively small fusion residue cross-sections. Here, a clear advantage of MNT reactions comes now into play. It arises from the broad excitation functions of MNT products which leads to a wide-band population of many different nuclides with sizeable yields in the same experiment, while fusion reactions are only selective on very few specific isotopes. In this region MNT seems indeed in competition with fusion reactions and might become an attractive option for future experiments.

\subsection{The region of neutron-rich $N=126$ nuclei}

\subsubsection{Model predictions}

This region is of great interest to understand the astrophysical r-process path which proceeds on the neutron-rich side of the nuclide chart and starts to traverse very neutron-rich, still unknown territory above the Sn region. So far, r-process nuclei are produced in the lab in fragmentation and fission reactions. But in the course of new model calculations, MNT reactions are suggested as an alternative because they reveal cross-sections which are comparable, or even larger than those of fragmentation reactions. The DNS as well as the Langevin model suggest to use targets close to this region like ${ }^{208} \mathrm{~Pb}$ or ${ }^{198} \mathrm{Pt}$, but differ in the choice of the projectile. The Langevin model obtains the largest cross-sections with heavy beams like ${ }^{136} \mathrm{Xe}$, which have similar $A / Z$ as the target nuclei, while the DNS model pleads for neutronrich but lighter projectiles such as ${ }^{48} \mathrm{Ca}$ or ${ }^{64} \mathrm{Ni}$. As discussed earlier, this reflects the stronger dominance of the Coulomb force over the nuclear force in the DNS approach making very heavy systems unfavorable. Figures 26 and 27 show respective calculations from the DNS $[28,35]$ and the Langevin model $[26,41]$. In the ${ }^{136} \mathrm{Xe}+{ }^{208} \mathrm{~Pb}$ reaction the Langevin model predicts larger cross-sections for production

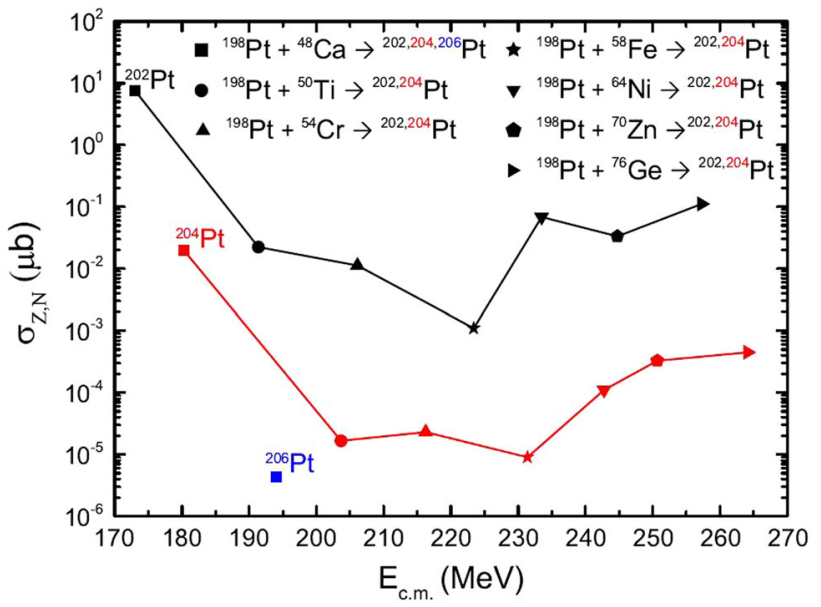

Fig. 26 DNS model calculations of the maximal MNT cross-sections [35] for the neutron-rich platinum isotopes ${ }^{202} \mathrm{Pt}$ (black symbols), ${ }^{204} \mathrm{Pt}$ (red symbols), ${ }^{206} \mathrm{Pt}$ (blue symbols) produced in collisions of the indicated projectiles with ${ }^{198} \mathrm{Pt}$ targets. The reaction products result from On evaporation channels

of neutron-rich Pt isotopes than the DNS model predicts for the reactions indicated in Fig. 26. However, the theoretical cross-sections in Ref. [41] are in better agreement with the data on the neutron-deficient side of the mass distribution for each isotope. Therefore, the cross-sections for neutron-rich isotopes might be overestimated in Fig. 27 [260].

Interesting is another approach which is suggested by the DNS model [28]. MNT reactions in ${ }^{48} \mathrm{Ca}+{ }^{238} \mathrm{U}$ are suggested to populate neutron-rich nuclei along $N=126$. The idea behind it is that the nucleon flow in MNT reactions is oriented along the stability line. This means that with $U$ targets, one can benefit from the preferred south-west flow of nucleons from target to projectile to populate isotopes along $N=126$. Indeed, the production of primary neutronrich products occurs with large cross-sections. However, they have enough excitation energy to evaporate at least three neutrons. The neutron evaporation shifts the isotopic distributions to the left and reduces the yields of neutron-rich nuclei of interest. With Pt targets the population of these nuclei depends on the rather suppressed flow in south-east direction, but Pt isotopes are produced "cold" which provides a gain for the yields of neutron-rich isotopes. Figure 28 shows the DNS model calculations for Pt nuclei produced in the MNT reaction ${ }^{48} \mathrm{Ca}+{ }^{238} \mathrm{U}$. The primary isotopic distributions are now shifted toward the neutron-rich side. After neutron evaporation, for example, ${ }^{204} \mathrm{Pt}$ is produced with about 50 times smaller cross-section $(0.4 \mathrm{nb})$ in the $\mathrm{Ca}+\mathrm{U}$ reaction than in the $\mathrm{Ca}+\mathrm{Pt}$ reaction $(20 \mathrm{nb})$. It is up to future experiments to verify this result of the model.

Cross-section calculations for nuclei along $N=126$ were also performed with other models $[45,47,50]$. For example, Zhu et al. investigated MNT reactions in various heavy sys- 

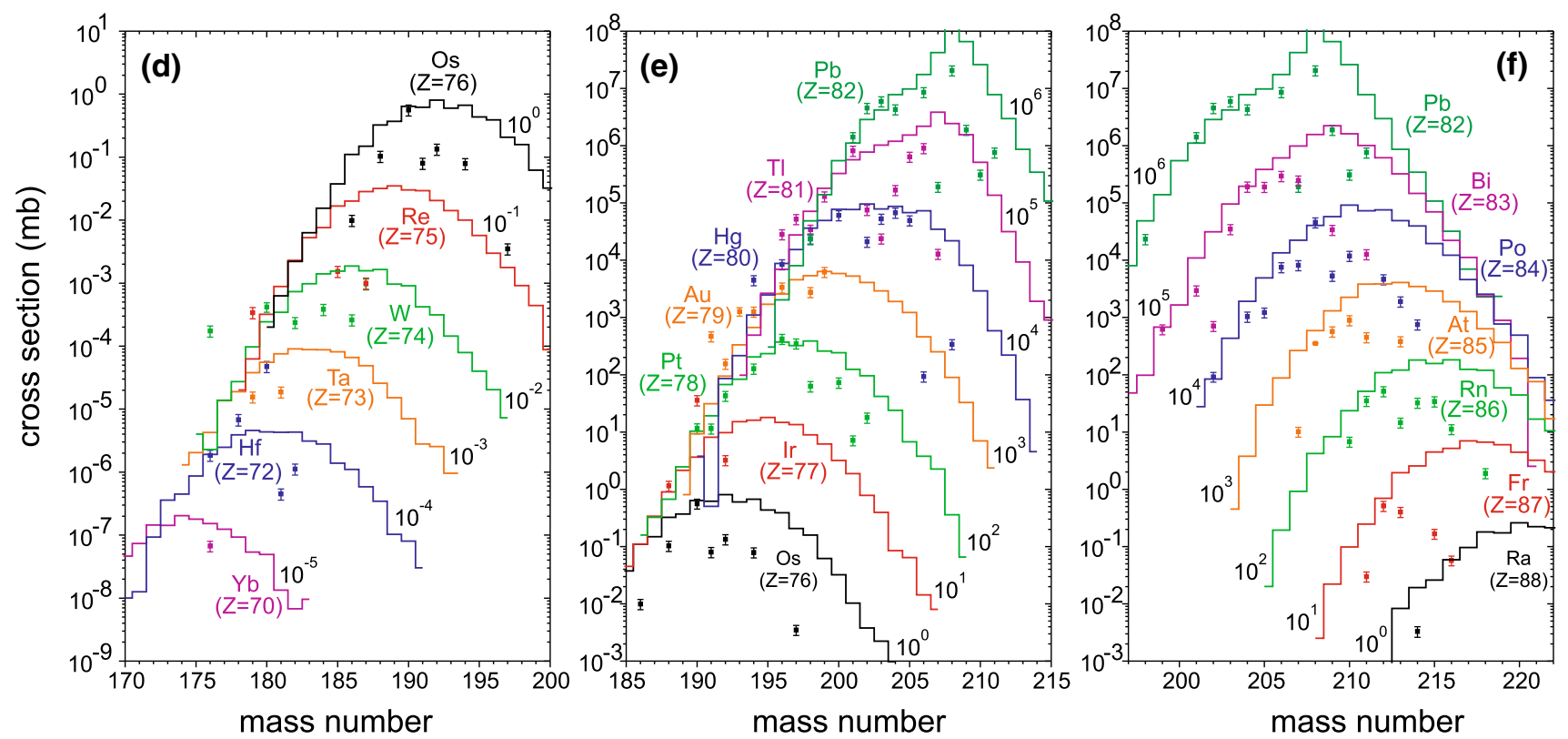

Fig. 27 Comparison of calculated (histograms, Langevin model [41]) and measured [260] (symbols) cross-sections for ${ }^{136} \mathrm{Xe}+{ }^{208} \mathrm{~Pb}$ at $E_{\mathrm{c} . \mathrm{m} .}=450$ $\mathrm{MeV}$

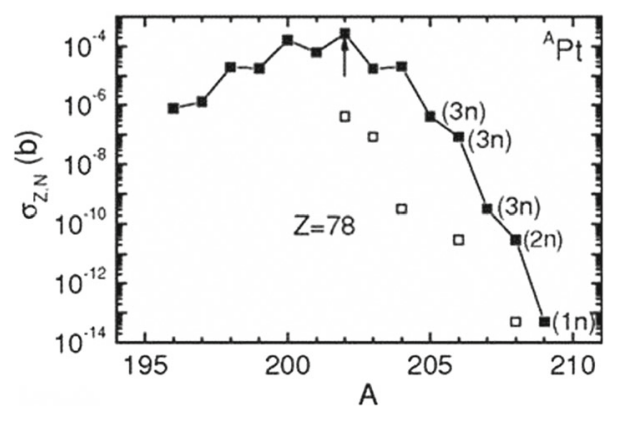

Fig. 28 DNS model calculations of MNT cross-sections for platinum isotopes produced in the ${ }^{48} \mathrm{Ca}+{ }^{238} \mathrm{U}$ reaction [28]. The yields of primary and secondary isotopes are shown by closed and open squares, respectively. Neutron evaporation channels for neutron-rich primary isotopes are indicated. The heaviest known isotopes are marked by arrows

tems like $\mathrm{W}+\mathrm{U}$ or $\mathrm{Xe}+\mathrm{Pt}$ by using the model which is based on the DNS approach and the isospin-dependent quantum molecular dynamics model [50]. The results of the various models are quite different and can vary by several orders of magnitude for the same isotope [50,261]. The values in Table 1 give an impression. In Ref. [41], the nucleon transfer before reaching thermal equilibrium was taken into account. This results in larger cross-sections than in Ref. [26]. Also the nucleon transfer rates and relevant degrees of freedom were specified in a more refined way. Though the existing models describe well the maxima of isotopic distributions, there is a large uncertainty in the slope on the right-hand side of the isotopic distributions. Therefore, the experiments on the production of neutron-rich isotopes with $N>126$ are desirable in order to reduce the theoretical uncertainties
Table 1 Predicted cross-sections of ${ }^{204} \mathrm{Pt}$ isotopes produced in MNT reactions with the denoted collision systems. The results originate from different theoretical models

\begin{tabular}{lll}
\hline & Cross-section & References \\
\hline${ }^{136} \mathrm{Xe}+{ }^{208} \mathrm{~Pb}$ & $8 \mu \mathrm{b}$ & {$[26]$} \\
${ }^{136} \mathrm{Xe}+{ }^{208} \mathrm{~Pb}$ & $200 \mu \mathrm{b}$ & {$[41]$} \\
${ }^{48} \mathrm{Ca}+{ }^{196} \mathrm{Pt}$ & $20 \mathrm{nb}$ & {$[35]$} \\
${ }^{48} \mathrm{Ca}+{ }^{238} \mathrm{U}$ & $0.4 \mathrm{nb}$ & {$[28]$} \\
\hline
\end{tabular}

and define the most efficient reactions for production of new neutron-rich nuclei with $N>126$.

\subsubsection{Experimental state of the art}

The population of neutron-rich nuclei along $N=126$ in MNT reactions was already studied in different experimental approaches. One can roughly distinguish them in experiments which identified the heavy target-like products directly [260,262-264] and in experiments which detected the projectile-like products and deduced from them the isotopic distributions of the complementary target-like reaction partners [265]. The results of the experiments which performed direct isotope identification reveal an astoundingly uniform picture, despite their different experimental approaches and collision systems. Krolas et al. started their first experiments about 15 years ago applying collisions of ${ }^{58,64} \mathrm{Ni}+{ }^{208} \mathrm{~Pb}$ at Legnaro [262,263]. In these "thick-target experiments" the reaction products were stopped in the target and their yield distributions were established from in- 


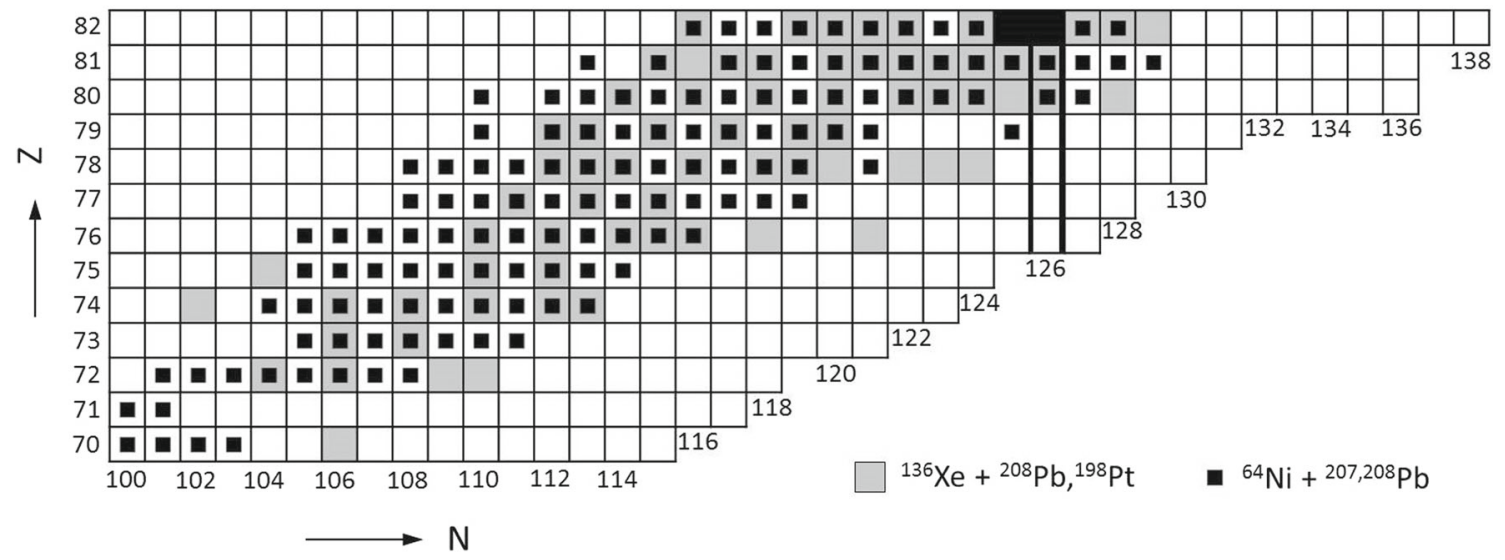

Fig. 29 Section of the chart of nuclides showing MNT products in the region below $\mathrm{Pb}$ which were populated in different experiments. The gray squares represent MNT products observed in reactions of ${ }^{136} \mathrm{Xe}$ beams with ${ }^{208} \mathrm{~Pb}$ and ${ }^{198} \mathrm{Pt}$ targets $[260,262,264]$. The small black

beam and off-line $\gamma$ spectroscopy. Actually, these experiments aimed for spectroscopic studies of the MNT products, which are usually created in excited energy and spin states, by applying the $\gamma-\gamma$ coincidence technique. Due to the stopping of the produced nuclei in the thick target foils, the emitted $\gamma$ rays are not affected by Doppler broadening, which enables high-resolution spectroscopy.

More recent studies were performed at Argonne National Laboratory (ANL) and by ourselves at GSI Helmholtz Centre. The ANL scientists investigated the ${ }^{136} \mathrm{Xe}+{ }^{208} \mathrm{~Pb}$ reaction in thick-target experiments and identified a broad region of projectile-like and target-like MNT products from $Z=48$ to $Z=88$ in off-line $\gamma$ ray spectroscopy [260]. At GSI, we studied the reaction ${ }^{64} \mathrm{Ni}+{ }^{207} \mathrm{~Pb}$ [264] with the velocity filter SHIP to separate the target-like MNT products which were emitted to zero degrees. Isotope identification was performed in the focal plane of the velocity filter by $\gamma$ spectroscopy. We want to stress that all experimental approaches used gamma spectroscopy to identify the target-like nuclei because it is presently the only method to identify heavy, low-energetic beta emitters. It is also noteworthy, that no new isotopes were observed in any of these experiments and that the lowest limit cross-sections were between $(1-10) \mu \mathrm{b}$ which is definitely not sensitive enough to advance deeper toward new neutron-rich nuclei. Figure 29 gives an overview on the isotopes with $Z=70-82$ which were so far produced in MNT reactions and directly identified. One can notice that the observed nuclei are still many neutrons far from the most neutron-rich known isotopes of the respective elements. The so far heaviest isotope, ${ }^{202} \mathrm{Pt}$, which was observed in MNT reactions was measured in Ref. [260].

Figure 30 shows measured MNT cross-sections of Os, $\mathrm{Ir}, \mathrm{Pt}, \mathrm{Au}, \mathrm{Hg}$ and $\mathrm{Tl}$ isotopes produced in collisions of squares indicate MNT products from the reactions ${ }^{64} \mathrm{Ni}+{ }^{207,208} \mathrm{~Pb}$. The limits of the chart given on the neutron-rich side correspond to the limits of the current Karlsruhe chart of nuclides of 2018

Nihbox $\mathrm{Pb}$ and $\mathrm{Xe}+\mathrm{Pb}$. Notably, the maximum crosssections reached in the different experiments are very similar and amount to several millibarns. But the isotopic distributions measured with ${ }^{136} \mathrm{Xe}$ beams are clearly shifted toward the neutron-rich side. Since isotopes in this area are usually produced in fragmentation reactions, we show in Fig. 30 also measured fragmentation cross-sections [266-270]. Interesting is that the MNT cross-sections are indeed similar or even larger than fragmentation cross-sections, like predicted by the models. And the discrepancy between MNT and fragmentation appears to increase toward the neutron-rich side to the advantage of MNT reactions. Also, a trend is indicated that MNT cross-sections increase with respect to fragmentation cross-sections toward smaller proton numbers. This trend was particularly observed by Watanabe et al. who investigated projectile-like MNT products from ${ }^{136} \mathrm{Xe}+{ }^{198} \mathrm{Pt}$ collisions at the VAMOS spectrometer at GANIL [265]. VAMOS was positioned at an angle of $30^{\circ}$, close to the grazing angle and supplemented by the EXOGAM array [271,272] around the target to measure prompt $\gamma$ rays. The $A$ and $Z$ resolutions were $\Delta A / A=1 / 200$ and $\Delta Z / Z=1 / 60$, allowing for identification of Xe-like fragments. The isotopic distributions of target-like MNT products were then deduced from the measured distributions of projectile-like nuclei.

So far, the results of all direct and indirect studies of MNT products below $\mathrm{Pb}$ are in good agreement and indeed indicate that MNT cross-sections overtake fragmentation crosssections toward the neutron-rich side and toward smaller proton numbers of the reaction products. But at this point it is important to consider that the experimental feasibility is not only determined by cross-sections but finally by the resulting yields which depend on experimental conditions like beam intensity, applicable target thickness and efficiency of the 

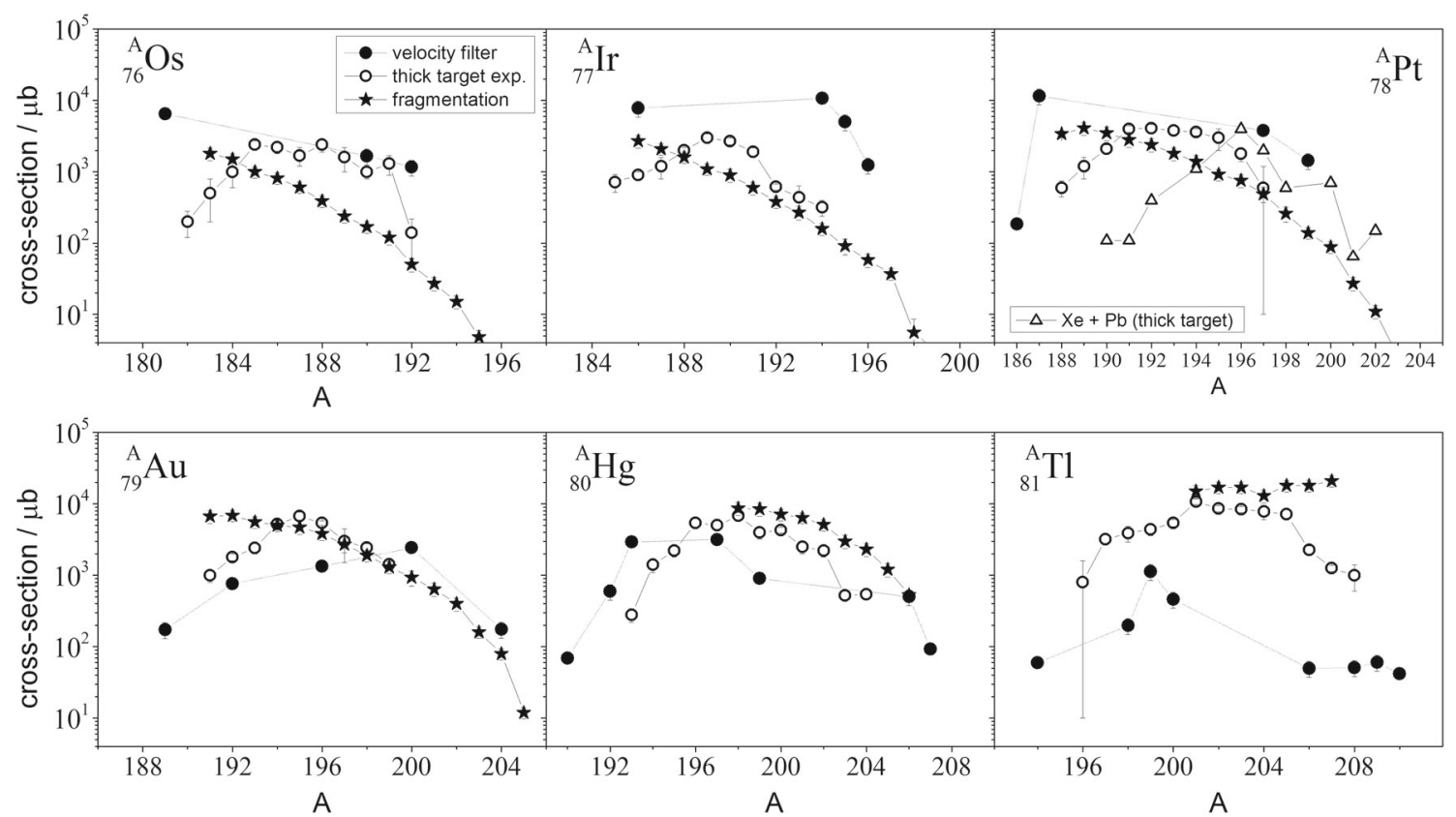

Fig. 30 Cross-sections of $\mathrm{Os}, \mathrm{Ir}, \mathrm{Pt}, \mathrm{Au}, \mathrm{Hg}$ and $\mathrm{Tl}$ isotopes produced in MNT reactions. Full circles represent the data from ${ }^{64} \mathrm{Ni}+{ }^{207} \mathrm{~Pb}$ collisions measured with the velocity filter SHIP at GSI [264]; the denoted cross-section values are corrected for the angular efficiency of SHIP, which was $0.5 \%$ in this case. Open circles denote data from a thick target experiment with ${ }^{64} \mathrm{Ni}+{ }^{208} \mathrm{~Pb}$ performed at Legnaro by Krolas et al. [262]. Open triangles represent the data on Pt isotopes measured by Barrett et al. at ANL in ${ }^{136} \mathrm{Xe}+{ }^{208} \mathrm{~Pb}$ collisions, equally in a thick-

experimental setup. And these are quite different in MNT and fragmentation reactions. In fragmentation reactions at relativistic energies, targets of $10 \mathrm{~g} / \mathrm{cm}^{2}$ can be used, while in MNT reactions at the Coulomb barrier target thicknesses are about $1 \mathrm{mg} / \mathrm{cm}^{2}$. In Fig. 31, we show the expected yields for MNT and fragmentation products, taking the cross-sections from Fig. 30 and using the parameters for beam current and target thicknesses given in the figure caption. Note that the yields in Fig. 31 are given at the target and do therefore not yet include the efficiencies of the experimental setups which can vary strongly between the different experiments. The overall trend indicates that the expected yields seem in favor of fragmentation reactions, mainly given by the possibility to use 10,000 times thicker targets. Apart from this, projectile fragmentation reactions combine further advantages, which make them much more efficient than MNT reactions: (i) projectile fragments are emitted in a very narrow forward cone, enabling very efficient and fast In-flight separation, while MNT products are emitted in a wide angular range which makes their collection/separation ineffective; (ii) $A$ and $Z$ identification of the relativistic fragments can be performed with the $E-\Delta E-\mathrm{TOF}-B \rho$ method which makes identification independent of decay properties; also, the method is very sensitive and is in principle applicable for a single event. In target experiment [260]. The cross-sections measured in fragmentation reactions $[266,267]$ are shown by asterisks. In fragmentation reactions, isotopes with still several more neutrons were discovered so far (see chart in Fig. 29 and Refs. [268-270]), but they are not all displayed here. The $N=126$ shell for $\mathrm{Os}, \mathrm{Ir}, \mathrm{Pt}, \mathrm{Au}, \mathrm{Hg}$, and $\mathrm{Tl}$ is located at mass numbers $A=202,203,204,205,206$, and 207 in the respective distributions

contrast, the lack of appropriate and sensitive identification techniques for heavy MNT products is still a serious bottleneck which makes the method inefficient and cumbersome.

\subsection{Shell effects in MNT reactions}

Experiments on DIC, many of them performed in the 1980s, indicate that shell effects play an important role during the DNS evolution [62,86-92] and influence the yield distributions of deep-inelastic reaction products. For example, in the symmetric reaction system ${ }^{238} U+{ }^{238} U$ [93] one observes an unexpectedly intense diffusion of protons compared with other symmetric systems like ${ }^{208} \mathrm{~Pb}+{ }^{208} \mathrm{~Pb}$ [94]. The variances of mass distributions in ${ }^{154} \mathrm{Sm}+{ }^{154} \mathrm{Sm}$ and ${ }^{144} \mathrm{Sm}+{ }^{144} \mathrm{Sm}$ reactions strongly differ, which is explained in [95] by the large neutron binding energy in the latter reaction. The cross-sections for compound nucleus formation in the reactions ${ }^{100} \mathrm{Mo}+{ }^{100} \mathrm{Mo}$ and ${ }^{110} \mathrm{Pd}+{ }^{110} \mathrm{Pd}$ differ by four orders of magnitude, although the masses of the interacting nuclei differ by only 10 units $[96,97]$.

The asymmetric systems ${ }^{16} \mathrm{O}+{ }^{92} \mathrm{Mo}$ and ${ }^{52} \mathrm{Cr}+{ }^{56} \mathrm{Fe}$ [98] or ${ }^{40} \mathrm{Ar}+{ }^{232} \mathrm{Th}$ and ${ }^{32} \mathrm{~S}+{ }^{238} \mathrm{U}$ [90] lead to the same compound nuclei but result in different charge distributions of the reaction products, indicating different paths of evolu- 

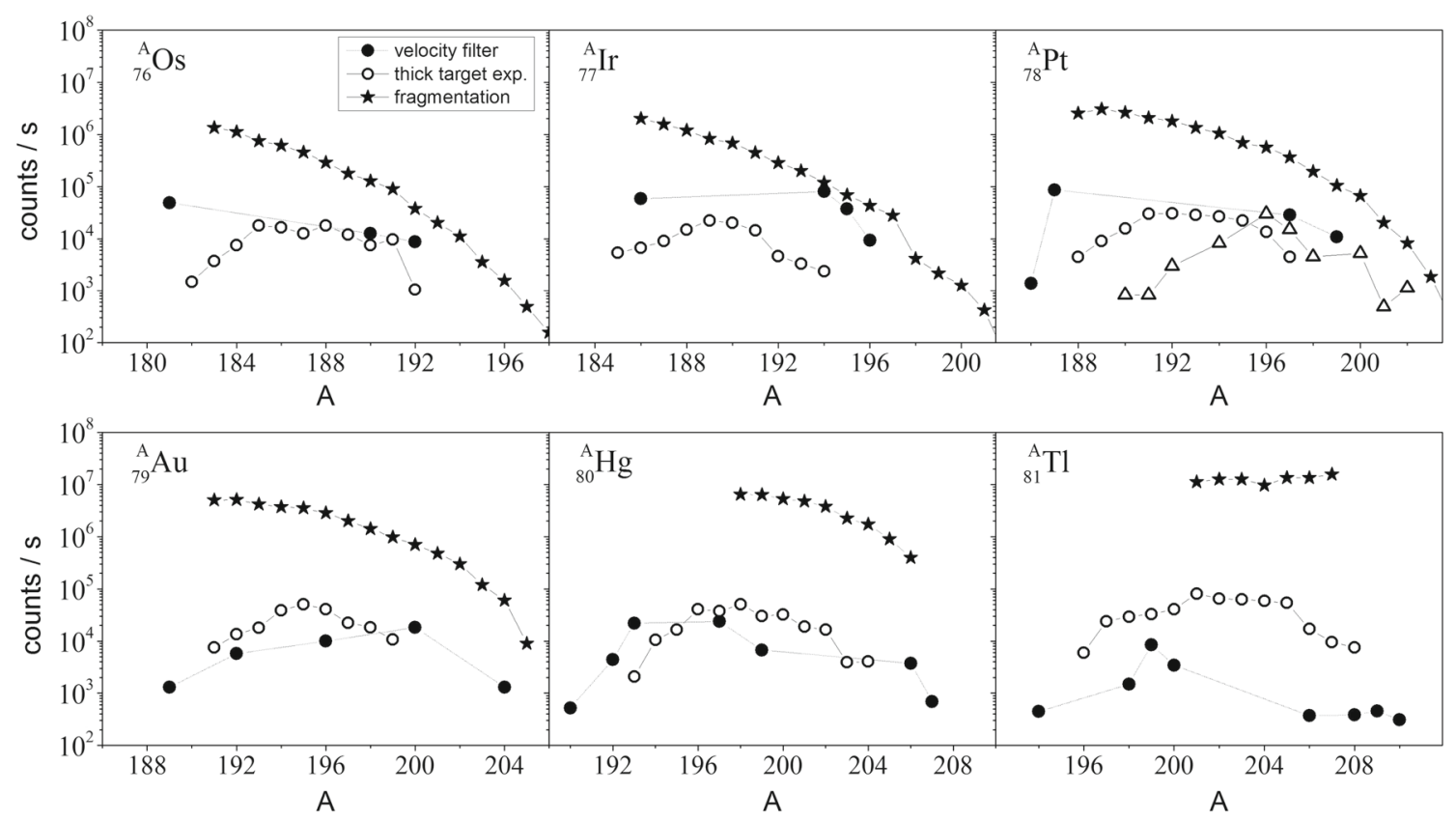

Fig. 31 Expected count rates per second of $\mathrm{Os}, \mathrm{Ir}, \mathrm{Pt}, \mathrm{Au}, \mathrm{Hg}$, and $\mathrm{Tl}$ isotopes produced in $\mathrm{MNT}$ reactions of $\mathrm{Ni}+\mathrm{Pb}$ and $\mathrm{Xe}+\mathrm{Pb}$. The count rates were calculated from the cross-sections in Fig. 30 by assuming the beam intensities and target thicknesses denoted in the follow- ing. Fragmentation: $\mathrm{I}=10^{10} / \mathrm{s}, \mathrm{d}=5 \mathrm{~g} / \mathrm{cm}^{2}$; MNT: $\mathrm{I}=5 \times 10^{12} / \mathrm{s}$, $\mathrm{d}=500 \mu \mathrm{g} / \mathrm{cm}^{2}$. The count rates are given at the target and do not include possible losses which occur during separation and detection of the nuclei at the individual setups tion of the respective DNS. In the ${ }^{48} \mathrm{Ca}+{ }^{238} \mathrm{U}$ reaction, a large mass variance is observed along with a small change in the mean mass number of the light fragment, but it is not the case in the ${ }^{40} \mathrm{Ca}+{ }^{238} \mathrm{U}$ reaction $[67,68]$.

Reactions with ${ }^{238} \mathrm{U}$ beams and targets of ${ }^{110} \mathrm{Pd}$ and ${ }^{124} \mathrm{Sn}$ were studied at GSI in the mid 1980s [273,274]. In both cases the bombarding energy was about $7 \%$ above the corresponding Coulomb barrier. Strong nuclear structure effects were not only found in the initial phase of the reaction with small energy transfer but also in the final phase with large energy dissipation. In ${ }^{238} \mathrm{U}+{ }^{110} \mathrm{Pd}$ reactions at $5.7 \mathrm{MeV} /$ nucleon, the nucleon flow toward symmetry was strongly oriented along the minimum of the driving potential in the early stage of the reaction until the $\mathrm{U}$-like MNT product reached the $Z_{P} \approx 82$, $N_{P} \approx 126$ shells and the target-like nucleus, nearly simultaneously, reached the $Z_{T} \approx 56, N_{T} \approx 82$ shells. After this, mainly protons flow from the heavy partner to the lighter one by keeping the neutron numbers of the two nuclei close to magicity. In the initial phase of the reaction ${ }^{238} \mathrm{U}+{ }^{124} \mathrm{Sn}$ at $6.02 \mathrm{MeV} /$ nucleon, equal numbers of protons and neutrons were transferred. However, starting from $Z_{P} \approx 82$, $N_{P} \approx 135$ and $Z_{T} \approx 60, N_{T} \approx 83$, mainly proton transfer was observed as in the ${ }^{238} \mathrm{U}+{ }^{110} \mathrm{Pd}$ case. A periodic variation of the multiproton transfer probability as a function of the nuclear charges of the residual light nuclei was also observed in both reactions [274]. Thus, the neutron transfers in these studies reflect driving potentials towards the $N_{T}=82$ and
$N_{P}=126$ closed shells $[273,274]$. In other words, the driving potential carries information about the paths of evolution of the DNS. Maxima in the mass and charge yields arise from minima in the driving potential and are caused by shell effects in the DNS nuclei $[20,29,160,167]$.

Also in the new generation of theoretical and experimental studies shell effects are discussed as an important factor for the yield distributions of MNT products. In the Langevin model, shell effects in very heavy systems like ${ }^{238} \mathrm{U}+{ }^{248} \mathrm{Cm}$ lead to a pronounced minimum at $Z_{P}=82, N_{P}=126$ in the driving potential and enhanced cross-sections for $\mathrm{Pb}$-like MNT products. As a consequence, also the cross-sections of the complemenatary superheavy target-like MNT product $\left(Z_{T}=106\right.$ in the case of $\mathrm{U}+\mathrm{Cm}$ collisions) will be enhanced [33]. New experimental data on shell effects in heavy systems are still scarce. Our studies of ${ }^{238} \mathrm{U}+{ }^{238} \mathrm{U}$ collisions did not reveal sufficiently strong signatures for shell effects to draw convincing conclusions [75]. Only, the measured isotopic distributions indicate that MNT products within the environment of four protons around $\mathrm{Pb}$ are created with lower excitation energies than more distant nuclei. Similar effects, but more pronounced, we observed in collisions of ${ }^{58,64} \mathrm{Ni}+{ }^{207} \mathrm{~Pb}$ at the velocity filter SHIP [71]. The influence of shell effects, particularly of the closed neutron shell at $N_{T}=126$, on the excitation energy of the transfer products was seen at the lowest energies, directly at the Coulomb barrier and faded with increasing beam 
energy. Also, we observed in different collision systems $\left({ }^{58,64} \mathrm{Ni}+{ }^{207} \mathrm{~Pb},{ }^{48} \mathrm{Ca}+{ }^{238} \mathrm{U},{ }^{48} \mathrm{Ca}+{ }^{248} \mathrm{Cm}\right)$ at SHIP that the cross-sections of MNT products in the region around $\mathrm{Pb}$ decreased only slightly, and the slope became steeper when the products move away from the $Z \approx 82$ shell [275]. Similar observations are reported in Refs. [78,79,276-278] for the systems ${ }^{48} \mathrm{Ca}+{ }^{232} \mathrm{Th},{ }^{238} \mathrm{U},{ }^{244} \mathrm{Pu},{ }^{248} \mathrm{Cm}$ as well as for ${ }^{36} \mathrm{~S},{ }^{48} \mathrm{Te},{ }^{64} \mathrm{Ni}+{ }^{238} \mathrm{U},{ }^{136} \mathrm{Xe}+{ }^{208} \mathrm{~Pb},{ }^{88} \mathrm{Sr}+{ }^{176} \mathrm{Yb}$ and ${ }^{156,160} \mathrm{Gd}+{ }^{186} \mathrm{~W}$.

All these results suggest that a careful choice of the collision system seems very important to exploit shell effects for enhancing the cross-sections of exotic nuclei in distinct regions of the nuclide chart.

\subsection{Transfer-type reactions at intermediate energies}

Besides MNT reactions at low energies, heavy-ion collisions at intermediate energies is a well established method for the production of rare isotopes [279-291]. As shown in Refs. [64,292], in the ${ }^{209} \mathrm{Bi}+{ }^{136} \mathrm{Xe}$ and ${ }^{197} \mathrm{Au}+{ }^{208} \mathrm{~Pb}$ reactions at the lower boundary of the Fermi energy domain the total reaction cross-section is almost entirely accounted for by binary collisions, irrespective of a possible further disassembly of the two highly excited primary fragments. Dissipative binary dynamics at these bombarding energies has been also observed in Refs. [293-295]. The observed influence of the target isospin on the final isospin of the projectilelike fragments and broadening of the projectile-like fragments charge distributions with increasing energy dissipation is similar to that encountered in MNT reactions at lower bombarding energies up to a few $\mathrm{MeV} /$ nucleon above the Coulomb barrier. The transfer process is found in Refs. [296305] to be a very strong component for the projectile-like products in peripheral collisions. In the following collision systems nucleon pickup products have been observed among the products of projectile fragmentation reactions at bombarding energies above the Fermi energy:

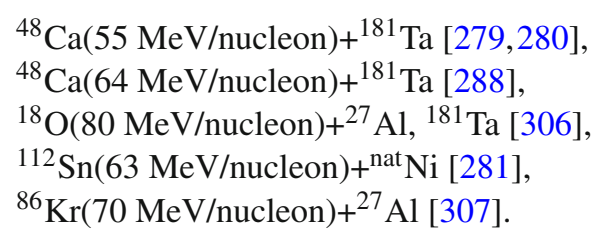

As well it was observed in reactions of ${ }^{40} \mathrm{Ca},{ }^{48} \mathrm{Ca},{ }^{58} \mathrm{Ni}$, ${ }^{64} \mathrm{Ni}$ beams at $140 \mathrm{MeV} /$ nucleon on ${ }^{9} \mathrm{Be}$ and ${ }^{181} \mathrm{Ta}$ targets [308]. As shown in Ref. [289], in the collisions of nuclei ${ }^{48} \mathrm{Ca}+{ }^{9} \mathrm{Be},{ }^{\text {nat }} \mathrm{W}$ at incident energy $142 \mathrm{MeV} /$ nucleon the yields of the most neutron-rich isotopes of light nuclei tend toward the $Q_{g g}$-systematics [57,62]. This allows us to assume the binary character of the interaction, contributing to the production of neutron-rich nuclei in the reactions ${ }^{48} \mathrm{Ca}+{ }^{9} \mathrm{Be},{ }^{\text {nat }} \mathrm{W}$. The larger yields of neutron-rich nuclei with ${ }^{\text {nat }} \mathrm{W}$ targets compared to ${ }^{9}$ Be targets indicates the strong contribution of reaction types other than fragmentation. In fragmentation reactions the sequential evaporation of light particles from the strongly excited nucleus leads to a somewhat uniform distribution of the final products which underlies the semi-empirical EPAX systematics based on the data from many high-energy experiments [309]. The disagreement of the yields of neutron-rich nuclei with the EPAX formula [309] supports the assumption that MNT binary reactions play an important role in the production of exotic nuclei even at quite high bombarding energy. The mechanism of this reaction seems to be the same like the mechanism of DIC. The collisions should occur with large angular momenta (large impact parameters) to supply small excitation energy in the neutron-rich products.

Nucleon transfer reactions at incident energies around $100 \mathrm{MeV} /$ nucleon must occur in nearly peripheral collisions to avoid high excitation in the DNS and fragmentation. The excitation energy of the DNS with an exotic nucleus $(Z, N)$ is $E^{*}(Z, N)$. Assuming thermal equilibrium, the excitation energy of the light nucleus with mass $A=Z+N$ in this DNS is $E_{P}^{*}(Z, N)=E^{*}(Z, N) A / A_{\text {tot }}$. It is clear that the formation probability of the DNS with exotic nucleus $(Z, N)$ increases with $E^{*}\left(Z_{i}, N_{i}, J\right)$. However, this increase is only possible up to the moment when $E_{P}^{*}(Z, N)$ becomes equal to the neutron separation energy $S_{n}(Z, N)$. Further increase of $E^{*}\left(Z_{i}, N_{i}, J\right)$ would lead to a strong loss of neutron-rich nuclei due to neutron emission. Taking $E_{P}^{*}(Z, N) \approx S_{n}(Z, N)$, we find the optimal value of $E^{*}\left(Z_{i}, N_{i}, J_{Z, N}\right)$ and the corresponding angular momentum $J_{Z, N}=R_{b}\left(2 \mu\left[E_{\text {c.m. }}-V\left(R_{b}, Z_{i}, N_{i}, J=\right.\right.\right.$ $\left.\left.0)-E^{*}\left(Z_{i}, N_{i}, J_{Z, N}\right)\right]\right)^{1 / 2}$. Only the partial waves with $J$ in the vicinity of $J_{N, Z}$ contribute to $\sigma_{Z, N}$. The collisions with $J<J_{N, Z}$ lead to $E_{L}^{*}(Z, N)>S_{n}(Z, N)$, and the contribution of collisions with $J>J_{N, Z}$ to $\sigma_{Z, N}$ decreases with increasing $J$ because the formation probability $Y_{Z, N, J}$ decreases. Therefore,

$\sigma_{Z, N} \approx \frac{\pi \hbar^{2}}{2 \mu E_{\mathrm{c} . \mathrm{m} .}} \Delta J\left(2 J_{N, Z}+1\right) Y_{Z, N, J_{Z, N}}$,

where $\mu$ is the reduced mass of the projectile-target combination and $\Delta J$ is the angular momentum interval above $J_{N, Z}$ which mainly contributes to the cross-section. In our calculation we set $\Delta J=20$ which corresponds to a change of the impact parameter of less than $0.2 \mathrm{fm}$ at the incident energies considered. Only a narrow region of partial waves contributes to the transfer cross-section, strongly selecting the initial condition.

To estimate $Y_{Z, N, J}$, the simple statistical method is used. The diffusion in mass and charge asymmetries is important in the DNS evolution. The simultaneous investigation of the diffusion in these collective coordinates allows us to calculate the formation probability $Y_{Z, N, J}$ of the DNS configu- 
ration. We assume that the average interaction time of two nuclei is much larger than the transient times in mass and charge asymmetries. We approximate the expression for the formation probability rate with the Kramers-type formula (33) [310] and obtain

$$
Y_{Z, N, J_{Z, N}} \approx 0.5 \exp \left(-\frac{\Delta B_{Z, N, J_{Z, N}}}{\Theta\left(Z_{i}, N_{i}\right)}\right)
$$

$\Delta B_{Z, N, J_{Z, N}}=U\left(R_{\mathrm{m}}, Z, N, J_{Z, N}\right)-U\left(R_{\mathrm{m}}, Z_{i}, N_{i}, J_{Z, N}\right)$ and the average interaction time $t_{0}=5 \mathrm{MeV}^{-1} \hbar \approx 3 \times$ $10^{-21} \mathrm{~s}$ of the two nuclei is assumed to be equal to the characteristic time of DIC. Note that the decay of the DNS from the initial configuration is the dominant decay channel here. As seen, the value of $\Delta B_{Z, N, J_{Z, N}}$ contains the corresponding $Q$-value to expect the $Q_{g g}$-systematics for the isotopic distribution.

The production cross-sections of neutron-rich isotopes calculated within our approach in the reactions ${ }^{48} \mathrm{Ca}+{ }^{181} \mathrm{Ta}$, ${ }^{n a t} \mathrm{~W}$ at intermediate incident energies are listed in Table 2. The comparison of these results with available experimental data has sense only for those neutron-rich isotopes which are mainly produced as primary products of binary reactions. To the yields of lighter isotopes there are contributions of fragmentation processes as well as of de-excitation by neutron emission of heavier primary isotopes. In order to describe these yields the extension of the present model is necessary.

The calculated results are in good agreement with most of the experimental data. This strongly supports the proposed model. Our calculations clarify that the MNT process is the main process which contributes to the total reaction yields of most of the exotic nuclei in the intermediate energy region. The observed new isotopes with neutron numbers larger than the projectile neutron number in the above named reactions are most probably transfer products from collisions at large angular momentum (peripheral collisions) leading to small excitation energy in the primary neutron-rich products. At $J<J_{Z, N}$, the primary neutronrich nuclei are excited and transformed into the secondary nuclei with less number of neutrons due to de-excitation by nucleon emission. The yield of these secondary nuclei follows the $Q_{g g}$-systematics as well because of the binary character of the reaction. Indeed, in Ref. [289] the $Q_{g g}$ systematics fit well the yields of various isotopes. The value of the angular momentum in the entrance channel governs the competition between fragmentation and massive transfer processes.

Instead of the $Q_{g g}$-systematics, a systematics based on the binding energy per nucleon of the neutron-rich isotope is suggested in Ref. [311]. The binding energy of the neutron-rich isotope correlates with the $Q_{g g}$-value because the mass excess of the conjugated heavy fragment weakly changes with mass number. The cross-sections calculated for very neutron-rich isotopes with the EPAX [309]
Table 2 Calculated [25] production cross-sections of nuclides in the indicated reactions at intermediate energies are compared with the available experimental data for the reactions ${ }^{48} \mathrm{Ca}(142 \mathrm{MeV} /$ nucleon) $+{ }^{\text {nat }} \mathrm{W}$ [289], ${ }^{48} \mathrm{Ca}(140 \mathrm{MeV} /$ nucleon $)+{ }^{181} \mathrm{Ta}$ [308] and ${ }^{48} \mathrm{Ca}(64$ $\mathrm{MeV} /$ nucleon $)+{ }^{181} \mathrm{Ta}$ [288]

\begin{tabular}{|c|c|c|c|c|}
\hline Reaction & $\begin{array}{l}E_{\mathrm{lab}} \\
\text { (MeV/nucleon) }\end{array}$ & Nuclide & $\sigma_{Z, N}$ (th.) & $\sigma_{Z, N}(\exp )$. \\
\hline \multirow[t]{9}{*}{${ }^{48} \mathrm{Ca}+{ }^{\text {nat }} \mathrm{W}$} & 142 & ${ }^{41} \mathrm{Si}$ & $4 \mathrm{nb}$ & $13_{-8}^{+6} \mathrm{nb}$ \\
\hline & 142 & ${ }^{42} \mathrm{Si}$ & $1.4 \mathrm{nb}$ & $0.9_{-0.3}^{+0.3} \mathrm{nb}$ \\
\hline & 142 & ${ }^{43} \mathrm{Si}$ & $4.4 \mathrm{pb}$ & $5_{-2}^{+2} \mathrm{pb}$ \\
\hline & 142 & ${ }^{44} \mathrm{Si}$ & $0.6 \mathrm{pb}$ & $0.7_{-0.5}^{+0.5} \mathrm{pb}$ \\
\hline & 142 & ${ }^{46} \mathrm{Si}$ & $32 \mathrm{fb}$ & \\
\hline & 142 & ${ }^{36} \mathrm{Mg}$ & $12.4 \mathrm{nb}$ & $5_{-1}^{+1} \mathrm{nb}$ \\
\hline & 142 & ${ }^{37} \mathrm{Mg}$ & $123 \mathrm{pb}$ & $90_{-20}^{+30} \mathrm{pb}$ \\
\hline & 142 & ${ }^{38} \mathrm{Mg}$ & $7 \mathrm{pb}$ & $40_{-10}^{+10} \mathrm{pb}$ \\
\hline & 142 & ${ }^{40} \mathrm{Mg}$ & $0.13 \mathrm{pb}$ & \\
\hline \multirow[t]{32}{*}{${ }^{48} \mathrm{Ca}+{ }^{181} \mathrm{Ta}$} & 140 & ${ }^{38} \mathrm{Si}$ & $17 \mu \mathrm{b}$ & $\sim 4 \mu \mathrm{b}$ \\
\hline & 140 & ${ }^{40} \mathrm{Si}$ & $55.9 \mathrm{nb}$ & $\sim 100 \mathrm{nb}$ \\
\hline & 64 & ${ }^{42} \mathrm{Si}$ & $0.8 \mathrm{nb}$ & \\
\hline & 64 & ${ }^{44} \mathrm{Si}$ & $0.4 \mathrm{pb}$ & \\
\hline & 64 & ${ }^{46} \mathrm{Si}$ & $24 \mathrm{fb}$ & \\
\hline & 64 & ${ }^{36} \mathrm{Mg}$ & $7.1 \mathrm{nb}$ & \\
\hline & 64 & ${ }^{38} \mathrm{Mg}$ & $4 \mathrm{pb}$ & $\sim 35 \mathrm{nb}$ \\
\hline & 64 & ${ }^{40} \mathrm{Mg}$ & $75 \mathrm{fb}$ & \\
\hline & 64 & ${ }^{41} \mathrm{Al}$ & $73 \mathrm{pb}$ & $\sim 8 \mathrm{nb}$ \\
\hline & 64 & ${ }^{43} \mathrm{Al}$ & $40 \mathrm{fb}$ & \\
\hline & 64 & ${ }^{45} \mathrm{Al}$ & $0.1 \mathrm{fb}$ & \\
\hline & 64 & ${ }^{45} \mathrm{P}$ & $54 \mathrm{pb}$ & \\
\hline & 64 & ${ }^{47} \mathrm{P}$ & $0.5 \mathrm{pb}$ & \\
\hline & 64 & ${ }^{46} \mathrm{~S}$ & $25 \mathrm{nb}$ & \\
\hline & 64 & ${ }^{48} \mathrm{~S}$ & $22 \mathrm{pb}$ & \\
\hline & 64 & ${ }^{50} \mathrm{~S}$ & $50 \mathrm{fb}$ & \\
\hline & 64 & ${ }^{49} \mathrm{Cl}$ & $2.2 \mathrm{nb}$ & \\
\hline & 64 & ${ }^{51} \mathrm{Cl}$ & $1.6 \mathrm{pb}$ & \\
\hline & 64 & ${ }^{53} \mathrm{Cl}$ & $2 \mathrm{fb}$ & \\
\hline & 140 & ${ }^{50} \mathrm{Ar}$ & $346 \mathrm{nb}$ & $\sim 150 \mathrm{nb}$ \\
\hline & 64 & ${ }^{52} \mathrm{Ar}$ & $0.82 \mathrm{nb}$ & \\
\hline & 64 & ${ }^{54} \mathrm{Ar}$ & $0.71 \mathrm{pb}$ & \\
\hline & 64 & ${ }^{54} \mathrm{Ar}$ & $0.71 \mathrm{pb}$ & \\
\hline & 64 & ${ }^{53} \mathrm{~K}$ & $30.6 \mathrm{nb}$ & \\
\hline & 64 & ${ }^{55} \mathrm{~K}$ & $17.3 \mathrm{pb}$ & \\
\hline & 64 & ${ }^{57} \mathrm{~K}$ & $0.19 \mathrm{pb}$ & \\
\hline & 64 & ${ }^{59} \mathrm{~K}$ & $3 \mathrm{fb}$ & \\
\hline & 64 & ${ }^{56} \mathrm{Ca}$ & $7.9 \mathrm{nb}$ & \\
\hline & 64 & ${ }^{58} \mathrm{Ca}$ & $83 \mathrm{pb}$ & \\
\hline & 64 & ${ }^{60} \mathrm{Ca}$ & $0.16 \mathrm{pb}$ & \\
\hline & 64 & ${ }^{59} \mathrm{Sc}$ & $3.5 \mathrm{nb}$ & \\
\hline & 64 & ${ }^{61} \mathrm{Sc}$ & $28 \mathrm{pb}$ & \\
\hline
\end{tabular}


Table 2 continued

\begin{tabular}{lllll}
\hline Reaction & $\begin{array}{l}E_{\text {lab }} \\
(\mathrm{MeV} / \text { nucleon })\end{array}$ & Nuclide & $\sigma_{Z, N}$ (th.) & $\sigma_{Z, N}$ (exp.) \\
\hline 64 & ${ }^{63} \mathrm{Sc}$ & $0.12 \mathrm{pb}$ \\
64 & ${ }^{60} \mathrm{Ti}$ & $136 \mathrm{nb}$ \\
64 & ${ }^{62} \mathrm{Ti}$ & $1.6 \mathrm{nb}$ \\
64 & ${ }^{64} \mathrm{Ti}$ & $15 \mathrm{pb}$ \\
64 & ${ }^{66} \mathrm{Ti}$ & $0.12 \mathrm{pb}$ \\
\hline
\end{tabular}

model in the case of fragmentation of ${ }^{48} \mathrm{Ca}$ projectiles [25] are larger than the experimental ones. The EPAX model describes well the yields of the isotopes of $\mathrm{Mg}$ and $\mathrm{Si}$ with $N-Z<10$. It is apparent that in the binary reaction the projectile must be as close as possible to the region of the nuclide to be produced because in this case a smaller number of nucleons has to be transferred.

Since the predicted production cross-sections for the new exotic isotopes ${ }^{47} \mathrm{P},{ }^{51,53,55,57} \mathrm{Cl},{ }^{52,54} \mathrm{Ar},{ }^{56,58,60} \mathrm{Ca}$, ${ }^{59,61,63} \mathrm{Sc}$, and ${ }^{62,64,66} \mathrm{Ti}$ are larger than $0.1 \mathrm{pb}$, they can be synthesized and detected with present experimental possibilities. The predicted cross-sections seem to be optimistic, specially for the isotopes of $\mathrm{Ca}, \mathrm{Sc}$ and $\mathrm{Ti}$, in the sense that the predictions are done by assuming that the excitation energy of the DNS is divided proportionally to the mass numbers of the fragments. In transfer reactions the excitation energy would be preferentially generated in the primary pickup products with $N+Z>N_{i}+Z_{i}$. One should also mention that the production cross-section weakly depends on the bombarding energy. For example, in the reactions ${ }^{48} \mathrm{Ca}+{ }^{181} \mathrm{Ta}$ the cross-section at beam energy $64 \mathrm{MeV} /$ nucleon is about of 5\% larger than at $140 \mathrm{MeV} /$ nucleon. This is because of the very weak dependence of the ratio $\left(2 J_{Z, N}+1\right) / E_{\mathrm{c} . \mathrm{m}}$. on $E_{\mathrm{c} . \mathrm{m}}$. in Eq. (58).

One can see that the cross-sections in ${ }^{48} \mathrm{Ca}+{ }^{\text {nat }} \mathrm{W}$ reactions are larger than the corresponding cross-sections in reactions of ${ }^{48} \mathrm{Ca}+{ }^{181} \mathrm{Ta}$. Irradiating heavier targets by ${ }^{48} \mathrm{Ca}$ beams for producing neutron-rich isotopes, we gain in the $Q_{g g}$ value as well as in the value of $\Delta B_{Z, N, J_{Z, N}}$. Therefore, heavier targets are preferable for the production of neutron-rich nuclei. For example, replacing ${ }^{181} \mathrm{Ta}$ or ${ }^{n a t} \mathrm{~W}$ by ${ }^{232} \mathrm{Th}$ or ${ }^{238} \mathrm{U}$ or ${ }^{248} \mathrm{Cm}$, one can increase the yield of neutron-rich isotopes. This effect should be taken into consideration in the planned experiments. Summarizing, we see that MNT reactions can occur also at intermediate energies and overlap with the fragmentation reactions. This leaves the question which energy is optimal for the production of new isotopes in MNT reactions.
4.6 Incomplete fusion of light weakly bound nuclei

To complete our considerations about nucleosynthesis in transfer reactions, we will discuss here the situation if one of the reaction partners is a very light nucleus. Understanding fusion dynamics of light weakly bound nuclei is very important for describing astrophysical reactions important for nucleosynthesis [312]. The breakup mechanism of weakly bound nuclei is crucial to reveal the dynamics of fusion as well as the consequences of breakup $[313,314]$. There are three possibilities of reaction processes upon breakup of a weakly bound nucleus. The first is where no fragments are captured and is termed non-capture breakup (NCBU). The second is where not all fragments are captured, which is termed incomplete fusion. The final possibility is where the light nucleus is captured completely by the target nucleus and is termed complete fusion [315]. An important aspect is the interplay between breakup and other reaction processes like the transfer process. However, due to the transfer process's similarities with the ICF process it is hard to separate them from an experimental point of view as the fusion products from both processes are the same. Transfer can also cause the breakup of weakly bound nuclei during low-energy collisions [316-324].

Various theoretical approaches for understanding the incomplete-fusion process have been developed in the past, spanning a range of concepts and considerations, including breakup fusion, angular momentum window for incomplete fusion, promptly emitted particles, Fermi-jet, exciton, and moving source, thereby explaining the measured energy spectra and angular distribution of the emitted fragments and population of angular momentum in the compound nucleus [325]. New types of models have been used to address low-energy fusion dynamics of weakly bound nuclei, ranging from classical to quantum-mechanical methods. Reference [315] provides a critical survey of different theoretical approaches. New studies on the inclusive non-elastic breakup cross-section may provide a quantum-mechanical route to the calculation of the ICF cross-section of weakly bound nuclei [326,327]. Another interesting quantum-mechanical framework is the time-dependent wave-packet (TDWP) method [329,330]. This method calculates the incomplete- and complete-fusion cross-sections unambiguously [330], which is a challenge using the continuum discretized coupledchannels method [331-333]. The TDWP approach is currently undergoing further development to be implemented using a three-dimensional reaction model.

Some of the challenges of the quantum-mechanical models can be overcome via the use of the three-dimensional classical dynamical model [334-336]. This model is implemented using the PLATYPUS code [336], which uses classical trajectories in conjunction with stochastic breakup $[334,335]$. This is done through the input, which includes 
a breakup function [335,337], determined from sub-barrier breakup measurement [320,322], that undergoes MonteCarlo sampling [334,335]. This breakup function encodes the effect of the Coulomb and nuclear interactions that cause the breakup, making this approach a quantitative dynamical model for relating the sub-barrier NCBU to the above-barrier incomplete and complete fusion of weakly bound nuclei, rather than a breakup model [334-336]. In contrast to most existing models for incomplete fusion, PLATYPUS treats the dynamics of incomplete fusion and provides a number of differential cross-sections that are critical for understanding exclusive experimental data [338]. This approach has very recently been extended to the incomplete fusion of complex projectiles [339]. It is also important to note that this fusion model only works at energies above the Coulomb barrier between the projectile and target. This is due to the absence of quantum tunneling that is the primary way of fusion at suband near-barrier energies. There has been a recent attempt to amend this classical model by adding a correction at sub- and near-barrier energies, to take into account quantum tunneling. This was done by incorporating a tunneling factor based on the WKB approximation [340]. This improved the results outputted from the model, relative to experimental sub-barrier fusion measurements [340]. Additional modifications have recently been suggested for interpreting sub-barrier breakup measurements $[323,324]$. The role of (i) prompt and delayed, direct breakup, and (ii) transfer-triggered breakup modes on incomplete fusion of ${ }^{6} \mathrm{Li}+{ }^{209} \mathrm{Bi}$ collisions at energies above the Coulomb barrier has been addressed with an extended version of PLATYPUS [341]. For instance, prompt breakup happens in the instant the excitation of the ${ }^{6} \mathrm{Li}$ projectile is chosen to take place. At this point ${ }^{6} \mathrm{Li}$ is converted into its cluster fragments (alpha-deuteron) and then the fragments and target propagate according to the defined interactions between them [335]. Delayed breakup is induced by reaching the $1^{+}, 2^{+}$or $3^{+}$resonant states in ${ }^{6} \mathrm{Li}$, which then triggers the dissociation of ${ }^{6} \mathrm{Li}$ with the delay coming from the half-life of the resonant ${ }^{6} \mathrm{Li}$ state. The $3^{+}$resonant state has a much longer half-life than the $2^{+}$and $1^{+}$resonant states, so the ${ }^{6} \mathrm{Li}$ breakup takes place at the outgoing branch of its trajectory, far away from the target nucleus, not affecting fusion. So the effect of the $3^{+}$resonance on fusion can be neglected.

Figure 32 shows the incomplete-fusion excitation function in collisions of ${ }^{6} \mathrm{Li}+{ }^{209} \mathrm{Bi}$. A number of reaction processes contributes to the formation of incomplete-fusion products, the dominant process being the neutron-stripping channel (see solid and dashed lines) that involves the breakup (delayed and/or prompt) of the projectile-like nucleus ${ }^{5} \mathrm{Li}$ [341]. In contrast, most quantum-mechanical fusion calculations assume that the direct breakup of ${ }^{6} \mathrm{Li}$ into $\alpha$ and deuteron is the dominant incomplete-fusion channel, which is not the main observed breakup channel [322-324].
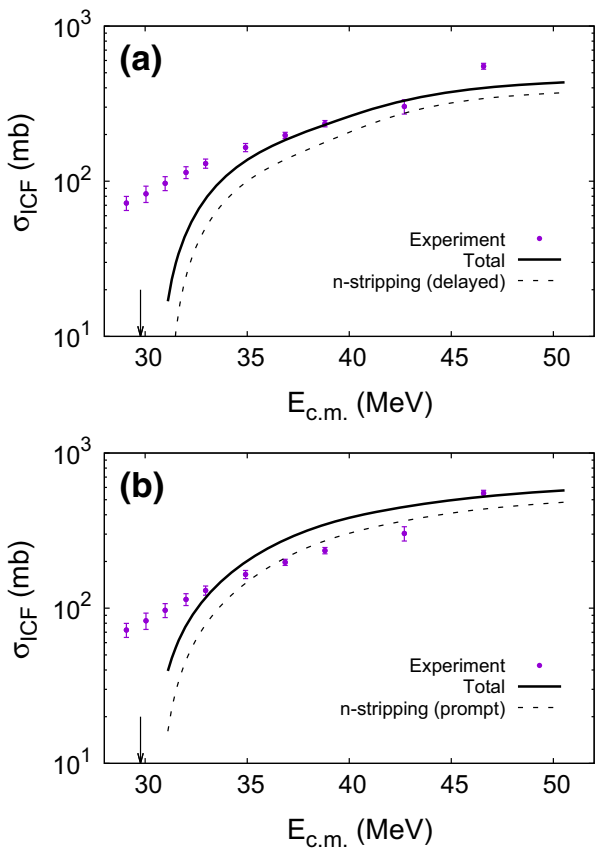

Fig. 32 Experimental incomplete-fusion cross-sections for ${ }^{6} \mathrm{Li}+{ }^{209} \mathrm{Bi}$ [342] are compared with PLATYPUS calculations at above-barrier (arrow) energies. Direct breakup as well as transfer-triggered breakup channels are included. a For delayed direct breakup of ${ }^{6} \mathrm{Li}$, delayed breakup of ${ }^{8} \mathrm{Be}$ after $d$-pickup, and delayed breakup of ${ }^{5} \mathrm{Li}$ after $n$-stripping. $\mathbf{b}$ The same but for prompt breakup processes. Clearly, the neutron-stripping process dominates the formation of ICF products (i.e., actinium and polonium isotopes)

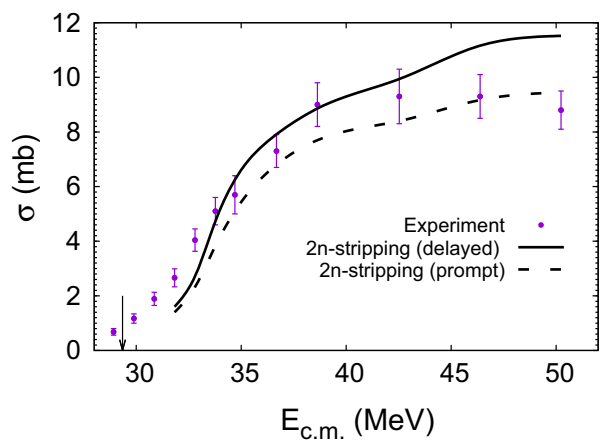

Fig. 33 Experimental production cross-sections of ${ }^{211} \mathrm{Bi}$ in ${ }^{7} \mathrm{Li}+{ }^{209} \mathrm{Bi}$ collisions [342] are compared with PLATYPUS calculations at abovebarrier (arrow) energies. The transfer-triggered breakup channels of ${ }^{5} \mathrm{Li}$ (either delayed or prompt) are included

The $2 n$-stripping channel is important for the production of ${ }^{211} \mathrm{Bi}$ in collisions of ${ }^{7} \mathrm{Li}+{ }^{209} \mathrm{Bi}$ at Coulomb energies [342], as shown in Fig. 33. This process also involves the breakup (delayed and/or prompt) of the projectile-like nucleus ${ }^{5} \mathrm{Li}$. Although the ${ }^{211} \mathrm{Bi}$ production is explained by this extended version of PLATYPUS [341], it fails in explaining the incomplete-fusion excitation function for ${ }^{7} \mathrm{Li}+{ }^{209} \mathrm{Bi}$, as shown in Fig. 34. This is due to the large contribution of the triton-transfer channel that cannot be treated within the 


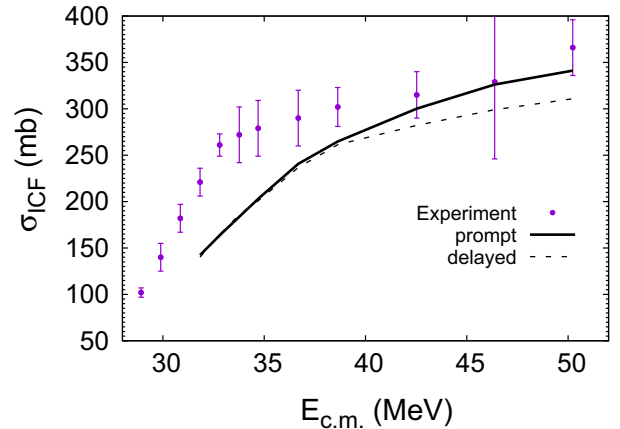

Fig. 34 Experimental incomplete-fusion cross-sections for ${ }^{7} \mathrm{Li}+{ }^{209} \mathrm{Bi}$ [342] are compared with PLATYPUS calculations at above-barrier energies. Theoretical calculations [341] include either prompt or delayed breakup of both ${ }^{7} \mathrm{Li}$ and projectile-like nuclei $\left({ }^{8} \mathrm{Be},{ }^{6} \mathrm{Li}\right.$ and $\left.{ }^{5} \mathrm{Li}\right)$ after single-particle transfer processes. The triton-transfer channel from the ${ }^{7} \mathrm{Li}$ ground state cannot be treated within the PLATYPUS model, but it is very important for the formation of specific incomplete-fusion products [328]
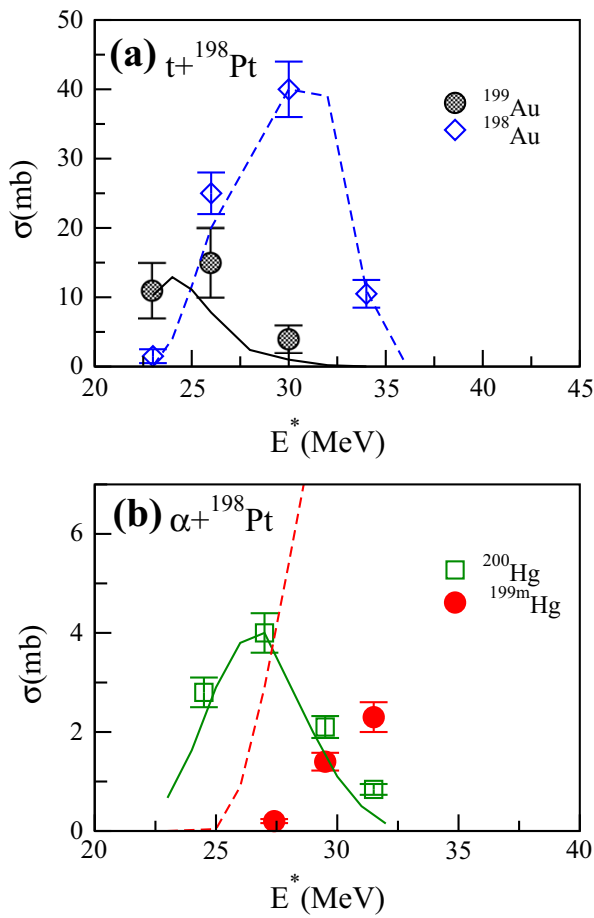

Fig. 35 Experimental production cross-sections (symbols) for indicated $\mathbf{a} \mathrm{Au}$ and $\mathbf{b} \mathrm{Hg}$ isotopes as a function of the excitation energy of the primary compound nucleus in the incomplete fusion of ${ }^{7} \mathrm{Li}+{ }^{198} \mathrm{Pt}$ are compared with PLATYPUS + PACE2 calculations (lines) [338]

PLATYPUS model. Very recent measurements [328] have confirmed the importance of this channel for the yield of specific incomplete-fusion products.

Figure 35 displays the production cross-section of some evaporation residues of (a) gold and (b) mercury isotopes in the incomplete fusion of ${ }^{7} \mathrm{Li}+{ }^{198} \mathrm{Pt}$ [338], after the direct breakup of ${ }^{7} \mathrm{Li}$ into triton and $\alpha$ fragments. Particle$\gamma$ coincidence measurements in conjunction with PLATY-
PUS+PACE2 calculations have provided insights into the formation mechanisms of these incomplete-fusion-evaporation residues [338], suggesting that the breakup fusion mechanism drives their formation. In case of ${ }^{199} \mathrm{Hg}$, the $\gamma$-ray transitions only above the $\left(13 / 2^{+}\right)$isomeric state were considered, therefore the measured cross-sections only provide a lower limit for this channel [338].

The present results for weakly bound stable ${ }^{6,7} \mathrm{Li}$ beams are useful for the theoretical developments, and also have implications in predicting production cross-sections of exotic nuclei with radioactive ion beams.

\section{Nucleosynthesis with radioactive ion beams}

The application of neutron-rich RIBs would allow one to use fusion-evaporation reactions, and thus to profit from the well defined forward oriented kinematics of the fusionevaporation residues. Besides, fusion reactions with neutronrich RIBs could be a further alternative to reach nuclei along the $N=126$ shell. Another possibility would be to use neutron-rich RIBs in MNT reactions [35,343]. Accelerator facilities which provide intense RIBs at Coulomb barrier energies are still in the process of arising. Nevertheless one can make some feasibility considerations based on model calculations. To prepare the discussion, we give in the next section an overview on the state of the art in RIB technologies and the yields which we could expect in such experiments.

\subsection{Radioactive ion beams: production and yields}

Principally, there are two different techniques of exotic ion beam production: the isotope separation on-line (ISOL) [344] and the In-flight method [345]. There are numerous review articles about RIB technologies to which we refer for details while we limit ourselves here to a short overview. The basic principles of the ISOL and In-flight technique are sketched in Fig. 36. The ISOL technique uses mostly proton beams in the $1 \mathrm{GeV}$ energy range to induce fragmentation or fission reactions in target nuclei of heavier elements up to uranium. Characteristic for the ISOL technique is the application of thick targets in which the reaction products are stopped. The targets are usually part of a combined target-ion-source system for production, extraction and ionization of the produced nuclei. From the target-ion-source, the ions are extracted with voltages of several $10 \mathrm{keV}$. A subsequent magnetic separator selects RIBs of the desired species. The ISOL technique is the oldest technology for RIB production [344]. A typical representative of an ISOL facility is the facility at CERN. The Inflight technique is in some sense complementary to the ISOL method. It uses the principle of projectile fragmentation or fission for which projectiles up to uranium are accelerated to energies of several $100 \mathrm{MeV} /$ nucleon up to $1 \mathrm{GeV} /$ nucleon 

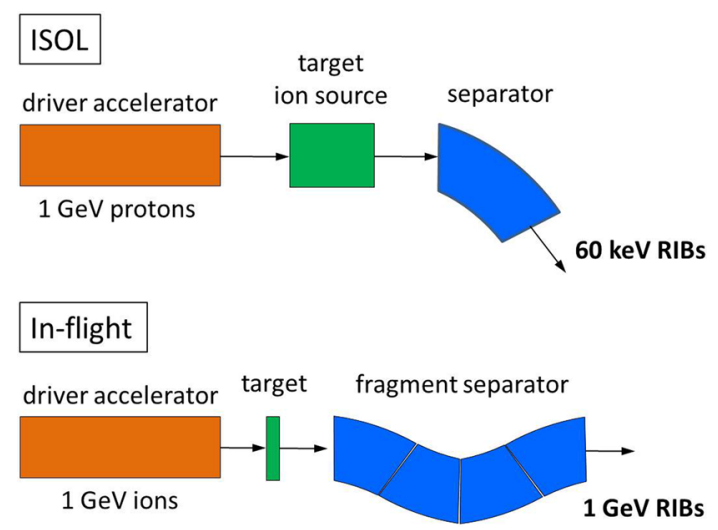

Fig. 36 The two basic methods for Radioactive Ion Beam (RIB) production. The ISOL (Isotope Separation Online) technique uses $\sim 1 \mathrm{GeV}$ proton beams to induce fragmentation or fission reactions in the target nuclei. The reaction products are stopped in the thick target and after extraction and ionization accelerated to energies of several $10 \mathrm{keV}$ by an electrostatic field (the here given value of $60 \mathrm{keV}$ corresponds to the CERN ISOLDE facility). A subsequent magnetic separator selects RIBs of the desired species. The In-flight technique uses the method of projectile fragmentaion or fission, where ion beams up to uranium are accelerated to energies of several $100 \mathrm{MeV} /$ nucleon up to $1 \mathrm{GeV} /$ nucleon and interact with a thin target from light elements like $\mathrm{Be}$ or $\mathrm{C}$. The reaction products emerge at relativistic energies and are strongly forward focused. RIBs of a certain species are then selected with a fragment separator

and collide with light target nuclei like beryllium or carbon. The created fragments are emitted with relativistic energies to a narrow forward cone and enter a subsequent fragment separator.

Due to the different techniques, the elemental and isotopic yields from ISOL and In-flight production are different (Figs. 37 [346], 38 [347]). The principal difference is that the ISOL technique cannot provide isotopes of all elements because the chemical properties of the elements determine how efficiently they can be ionized and extracted from the target-ion-source. Figure 37 reveals the gaps in the isotope (element) yields which emerge from this effect. The highest yields are obtained for heavy alkali elements and nobel gases. Also, the availability of very light RIBs $Z<\sim 25$ ) is quite restricted and their intensity is typically much higher in In-flight production. These restrictions do not concern the Inflight technique which can provide isotopes of all elements.

\subsection{How to get RIBs to Coulomb barrier energies?}

Fusion-evaporation reactions require RIBs at Coulomb barrier energy, which is around $5 \mathrm{MeV} /$ nucleon for asymmetric collision systems leading to superheavy nuclei. But neither the ISOL nor the In-flight technique can directly produce RIBs with these energies. Therefore, post-acceleration or deceleration, respectively, is necessary. In the following we discuss established and thinkable techniques and estimate the

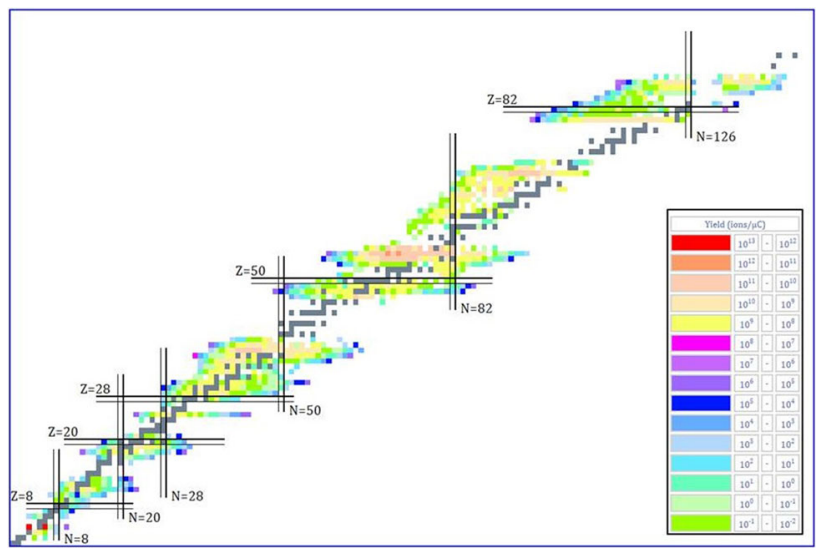

Fig. 37 Typical elemental and isotopic yields of RIBs produced with the Isotope Separation Online (ISOL) technique. The figure shows exemplary the presently available isotopes and their intensities at CERN ISOLDE [346]

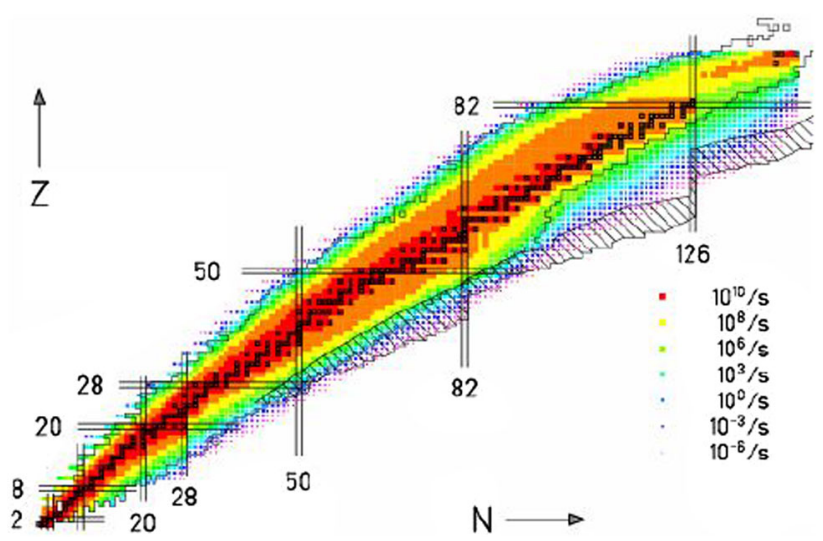

Fig. 38 Chart of nuclides with the expected isotopic yields of RIBs produced with the In-flight technique [347]

beam losses which are connected with them. The individual methods are depicted in Fig. 39.

\subsubsection{Post-acceleration of ISOL beams}

Radioactive ion beams produced with the ISOL method must be post-accelerated to bring them to Coulomb barrier energies (see scheme in Fig. 39). A classical example for such a facility is ISOLDE at CERN which delivers since recent years RIBs with energies between (5-10) MeV/nucleon by using the post-accelerators REX-ISOLDE [348] and HIE-ISOLDE [349]. For efficient post-acceleration, charge breeding is necessary. But before injection to the charge breeder, the mass separated RIBs with original charge state $1^{+}$are accumulated, cooled and bunched in a Penning trap. After extraction from the Penning trap, the ion bunches are injected to the charge breeder, where they are bombarded with electrons to further ionize them to higher charge states. This procedure is responsible for the main intensitiy losses of ISOL beams. 


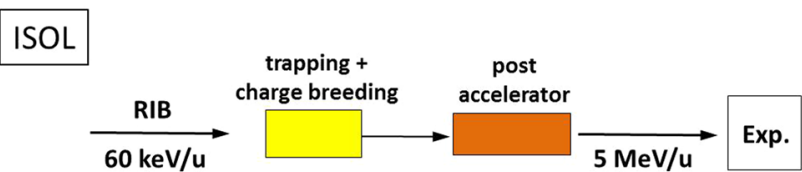

In-flight

(a)

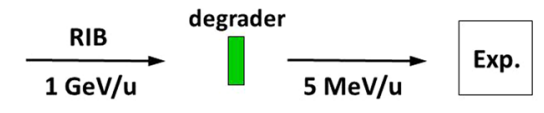

(b)

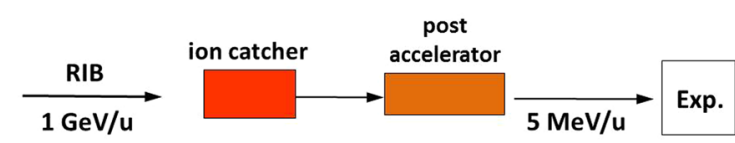

(c)

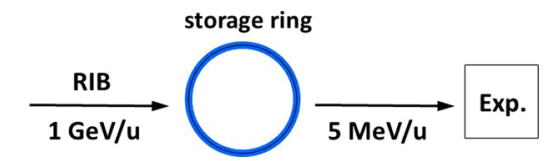

Fig. 39 Different techniques to obtain exotic ion beams at Coulomb barrier energies. For RIBs produced with the ISOL technique, postacceleration after charge breeding is applied. For high-energy RIBs produced with the In-flight technique, different methods can be used for deceleration: a application of degraders, $\mathbf{b}$ stopping in an ion catcher, followed by charge breeding and re-acceleration, $\mathbf{c}$ injection and deceleration in a storage ring

For example, the trapping and charge breeding system at ISOLDE has efficiencies between $5 \%$ and $20 \%$, depending mainly on the ion mass [350]. It reaches a maximum around $A=50$ and decreases toward larger masses. One has also to consider that cooling and charge breeding needs time. Cooling times are typically longer than $\sim 10 \mathrm{~ms}$. The breeding time varies between few milliseconds and $500 \mathrm{~ms}$, also depending mainly on the ion mass. Therefore only ions with sufficiently long half-lives survive the procedure. Roughly, the half-life of the nuclei should at least be on the order of seconds to avoid additional losses due to radioactive decays before reaching the production target. Postaccelerated ISOL beams are of good quality, having small emittance and energy spread.

\subsubsection{Deceleration of In-flight beams}

Deceleration with degraders The simplest method to slow down relativistic RIBs is the application of degrader foils. For RIBs with energies of several $100 \mathrm{MeV} / \mathrm{u}$, one needs degrader thicknesses of at least $10 \mathrm{~g} / \mathrm{cm}^{2}$. Interesting is to see the impact of such thick foils on beam emittance and energy spread. Respective studies were performed for the low-energy branch of the future Super-FRS facility $[351,352]$ at FAIR/GSI. Figure 40 shows the simulation
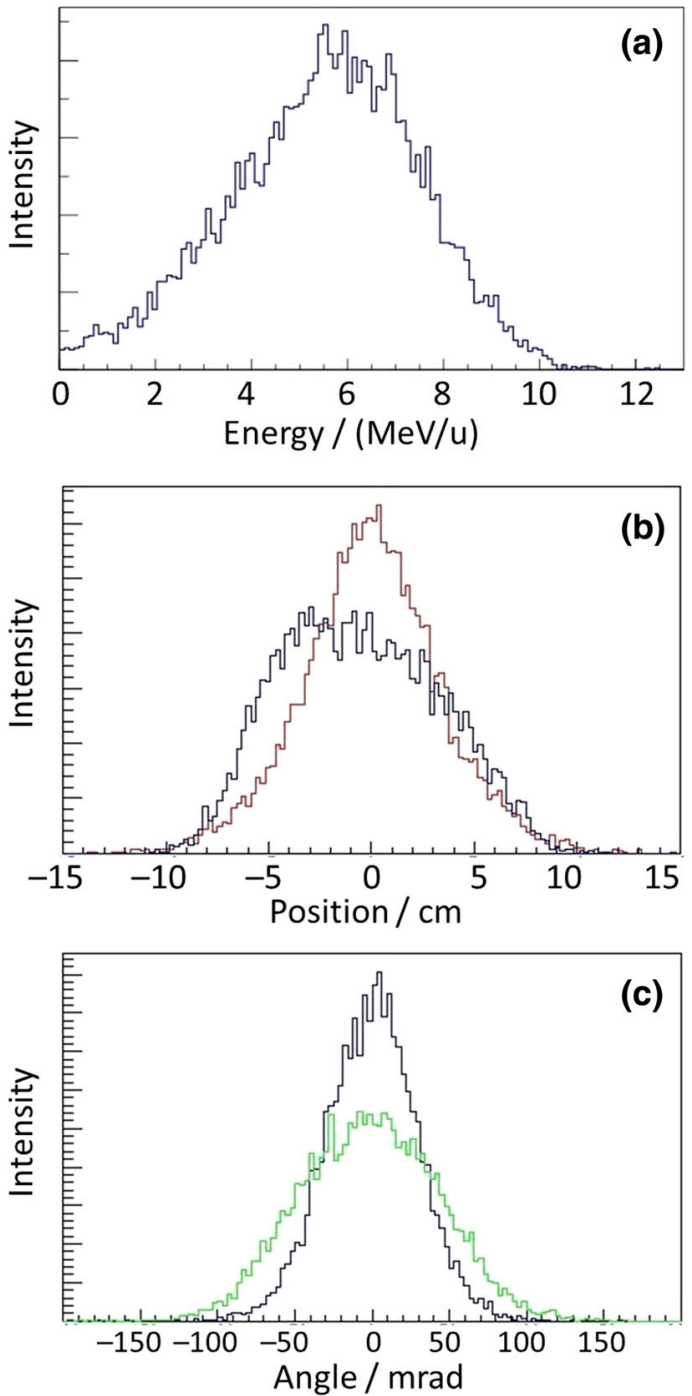

Fig. 40 Simulation of a beam energy distribution, $\mathbf{b}$ beam spot size and c opening angle distribution after decelerating a (radioactive) oxygen beam with degrader foils from $190 \mathrm{MeV} /$ nucleon to $6 \mathrm{MeV} /$ nucleon [353]. For details see text

results for the example of oxygen RIBs with an energy of $190 \mathrm{MeV} /$ nucleon [353]. In this example, four thinner degraders instead of a thick one were installed in different places along the beam line through the Super-FRS and subsequent energy buncher. The degraders were assumed as aluminum wedges with thicknesses of $3.7 \mathrm{~g} / \mathrm{cm}^{2}, 1.8 \mathrm{~g} / \mathrm{cm}^{2}$, $3.46 \mathrm{~g} / \mathrm{cm}^{2}$ and $0.63 \mathrm{~g} / \mathrm{cm}^{2}$ which decelerate the beam stepwise to $6 \mathrm{MeV} /$ nucleon. Figure 40a shows the energy distribution of the oxygen ions after the last degrader. It is peaked around $6 \mathrm{MeV} /$ nucleon with a full width at half maximum (FWHM) of $4 \mathrm{MeV} /$ nucleon $(\Delta E / E=67 \%)$. The excitation functions of hot fusion reactions leading to superheavy nuclei have relative widths of $\Delta E / E \approx 20 \%$ at FWHM which means that only $\sim 10 \%$ of the beam intensity can be effectively used for the synthesis reaction. Another factor 10 
of beam intensity is lost due to small angle scattering in the degraders in course of the deceleration process. Finally, the RIB intensity which is effectively available for the synthesis reaction is $\sim 1 \%$ of the original RIB intensity. Besides, the beam spot size and emittance are large: the beam diameter at the production target is $10 \mathrm{~cm}(!)$ and the opening angle is $100 \mathrm{mrad}$ (Fig. 40b, c). The deceleration with degraders is a fast method, where the lower time limit is only given by the flight time of the RIBs through the separator, but it leads to bad beam quality.

Gas stopping and post-acceleration Another possibility to decelerate In-flight RIBs is their stopping in a gas catcher and successive re-acceleration after extraction. This method provides decelerated RIBs of good quality. A respective technique has so far been developed at the Facility for Rare Isotope Beams (FRIB) at the National Superconducting Cyclotron Laboratory (NSCL) of Michigan State University [354]. The bottlenecks in such systems are the stopping and extraction efficiencies of the ion catcher and the efficiency of the charge breeding which is performed at NSCL with an electron beam ion source. With state-ofthe-art ion catchers, stopping and extraction efficiencies of some $10 \%$ can be reached. The charge breeder efficiencies are on the same scale. At NSCL it is planned to replace in the future the gas-stopping cell by a gas-filled reverse-cyclotron [354,355].

Deceleration in a storage ring Another imaginable possibility to obtain Coulomb barrier beams with good quality could be the deceleration of In-flight RIBs in a storage ring (Fig. 39). Already existing storage rings for exotic beams like the ESR [356] at GSI Helmholtzzentrum or HIRFL-CSR [357] in Lanzhou would be predestined for this method. The GSI ESR, for example, can decelerate relativistic ions down to energies of $4 \mathrm{MeV} /$ nucleon. During the deceleration process, beam cooling is necessary, which is performed by electron cooling. With this, the complete deceleration process takes about $10 \mathrm{~s}$, which means that the method is suitable for ions with lifetimes larger than roughly $10 \mathrm{~s}$. The bottlenecks in this method are beam losses during injection in the storage ring and during the deceleration and cooling process leading in worst cases to efficiencies on the $1 \%$ scale. But technical improvements are always possible.

If one carries the thought further, one could even imagine to let the decelerated RIBs circulate in the storage ring and let them traverse in each turn a thin target installed in the ring. At Coulomb barrier energies, the beam performs about $10^{5}$ turns per second in a ring with circumference of about $100 \mathrm{~m}$, like the ESR. This would effectively enhance the RIB intensity by the same factor $10^{5}$. There are so far no feasibility studies how realistic such a scenario could be. The critical point is the target, which leads to energy loss and deterioration of the beam emittance, followed by beam losses.
5.3 Superheavy nuclei from fusion reactions with RIBs?

\subsubsection{Expected cross-sections and yields}

Figure 41 shows the superheavy element region of the Chart of Nuclides with the presently known isotopes. Besides, it contains three areas of major interest which are still empty or not confirmed, respectively. One of these areas are the gaps between nuclei produced in cold and in hot fusion reactions. Filling these gaps would join the presently isolated region of relatively neutron-rich superheavy nuclei to the remaining, well established part of the nuclide chart. To fill these gaps, one does not even need RIBs because nearly all of them can be synthesized in fusion-evaporation reactions with stable beams, for example by using ${ }^{248} \mathrm{Cm}$ targets and the beams indicated on the right side of the chart. Another area of interest are the endpoint nuclei of the decay chains of superheavy isotopes from hot fusion reactions. Nuclei below $Z=112$ in these chains were so far never produced directly and their direct synthesis would substantiate their correct assignment as well as the one of their mother nuclei. And finally, there is the "island of stability" with the predicted but still not confirmed new spherical shell closures in the superheavy element region. Concerning the proton shell closure, the model predictions diverge: $Z=114,120$ or 126 are suggested, but the location of the neutron shell is found by most theoretical models at $N=184$ [358-362]. Figure 41 reveals that

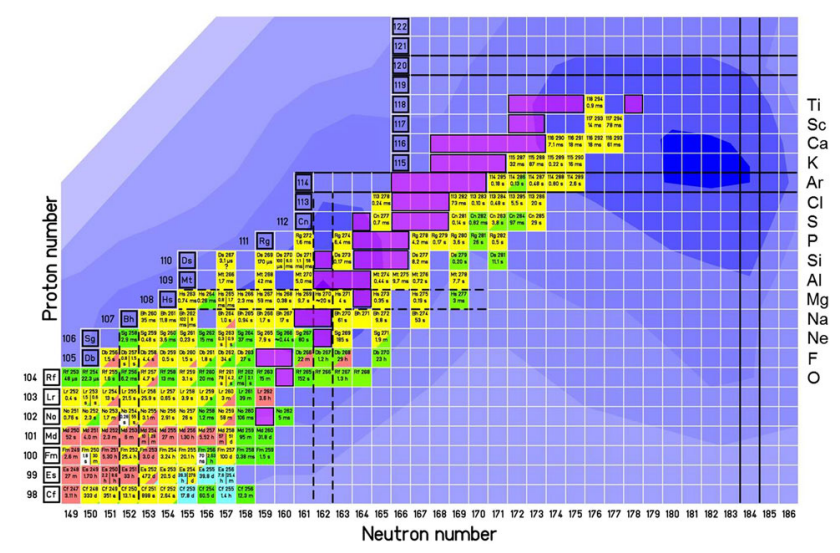

Fig. 41 Chart of Nuclides with the known isotopes of superheavy elements. The blue background represents shell correction energies (i.e. fission barriers) calculated in the macroscopic-microscopic model [358]. The chart contains three still empty, but very interesting regions: (i) the island of enhanced stability around $Z=114, N=184$, where spherical shell closures are expected; (ii) the endpoint nuclei of the decay chains of the most neutron-rich known isotopes, which were so far not produced directly and (iii) nuclei which fill the gaps between isotopes produced in hot and cold fusion reactions. To fill areas (i) and (ii), fusion reactions with radioactive ion beams or MNT reactions must be applied, while nuclei in area (iii) can be produced in fusion reactions with stable beams. The violet squares mark isotopes which can, for example, be produced in fusion reactions of ${ }^{248} \mathrm{Cm}$ targets with stable isotopes of the projectile nuclei indicated on the right side 
the most neutron-rich known isotopes are still seven neutrons away from $N=184$. The previous section revealed that MNT reactions are realistic to produce several endpoint nuclei of decay chains from isotopes produced in hot fusion reactions, but they will very likely not allow one to synthesize nuclei on the island of stability. There remains the question if fusion reactions with RIBs would allow one to access it.

To get now an idea about the yields of superheavy nuclei which can be expected from fusion reactions with RIBs, we start with some basic considerations: All experiments on superheavy element synthesis with stable beams revealed so far that complete-fusion reactions with as asymmetric as possible projectile-target combinations lead to the largest evaporation residue cross-sections [104,105], because in this case the Coulomb repulsion between the reaction partners is minimized. There is currently no serious reason to assume that the situation would change if radioactive projectiles are used instead of stable ones. Principally there are, like in fusion reactions with stable beams, two possible approaches: (i) cold fusion reactions using $\mathrm{Pb}$ or $\mathrm{Bi}$ targets and (ii) hot fusion reactions using actinide targets. In cold fusion reactions, the evaporation residues are quite neutron-deficient. Also, the experiments with stable beams revealed so far that nuclei above $Z=112$ are produced with significantly larger crosssections in hot fusion reactions. Therefore, we concentrate in the following representative examples on hot fusion reactions with ${ }^{248} \mathrm{Cm}$ targets, which is one of the heaviest and most neutron-rich available target material.

We first take a look on two representative model predictions of fusion-evaporation residue cross-sections. The first example in Fig. 42 shows DNS model cross-sections of Moscovium isotopes $(Z=115)$ from complete-fusion reactions of radioactive ${ }^{47} \mathrm{~K}$ beams with ${ }^{245,247,248} \mathrm{Cm}$ targets [363]. The most neutron-rich fusion products are ${ }^{291} \mathrm{Mc}$ and ${ }^{292} \mathrm{Mc}$, resulting from $4 n$ and $3 n$ evaporation channels. These are just the next neighbors of the already known isotope ${ }^{290} \mathrm{Mc}$ [364], and still seven neutrons far from the $N=184$ shell. The maximum cross-section is about $6 \mathrm{pb}$, which is on the same order as cross-sections measured for Mc isotopes with stable projectiles. It has to be remarked that also the adiabatic model finds very similar cross-sections for superheavy nuclei in the same region [26]. The second example (Fig. 43) shows excitation functions of the neutron-rich rutherfordium isotopes ${ }^{266,267} \mathrm{Rf}$ produced in collisions of ${ }^{22} \mathrm{O}$ beams with ${ }^{248} \mathrm{Cm}$ targets. These calculations were performed with the adiabatic model which predicts maximum cross-sections of $50 \mathrm{nb}$ and $5 \mathrm{nb}$, respectively [26]. The isotopes ${ }^{266,267} \mathrm{Rf}$ are the endpoint nuclei of the decay chains of ${ }^{282} \mathrm{Nh}$ [365] and ${ }^{291} \mathrm{Lv}$ [366] and were so far not produced directly.

The crucial parameter which decides about the experimental feasibility is the available RIB intensities. To get an impression, we show in Table 3 the expected average measurement times which would be necessary to produce one

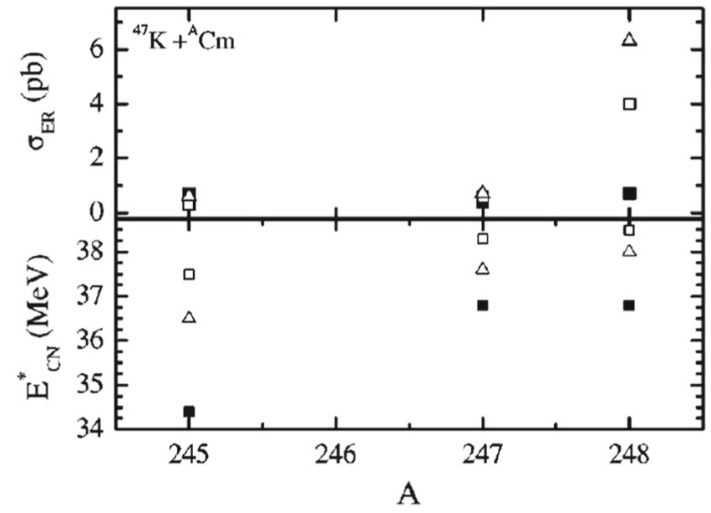

Fig. 42 DNS model calculations [363] of the maximal fusionevaporation residue cross-sections for $Z=115$ nuclei produced in collisions of neutron-rich ${ }^{47} \mathrm{~K}$ projectiles with ${ }^{245,247,248} \mathrm{Cm}$ targets (upper part). Different symbols belong to different excitation energies of the respective compound nuclei (lower part)

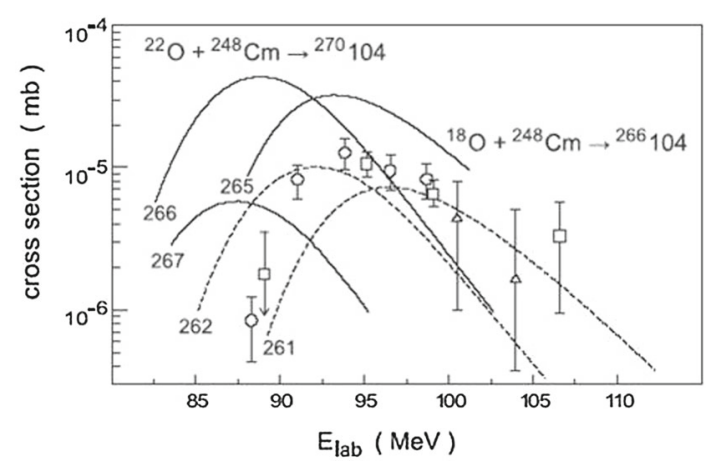

Fig. 43 Calculated [26] (lines) and experimental (symbols) excitation functions for the indicated fusion-evaporation residues with $\mathrm{Z}=104$ produced in collisions of stable and neutron-rich oxygen beams with ${ }^{248} \mathrm{Cm}$ targets

Table 3 Expected yields of the neutron-rich superheavy isotopes ${ }^{266} \mathrm{Rf}$ and ${ }^{291} \mathrm{Mc}$ produced in fusion-evaporation reactions of radioactive ${ }^{22} \mathrm{O}$ and ${ }^{47} \mathrm{~K}$ beams with ${ }^{248} \mathrm{Cm}$ targets. The isotope ${ }^{291} \mathrm{Mc}$, which has a proton number close to the possible shell closure at $Z=114$, is still 8 neutrons far from the predicted $N=184$ neutron shell closure. The presently available intensities of the RIBs and expectation time for observing 1 event are listed

\begin{tabular}{lll}
\hline Isotope & ${ }^{266} \mathrm{Rf}$ & ${ }^{291} \mathrm{Mc}$ \\
\hline Projectile & ${ }^{22} \mathrm{O}$ & ${ }^{47} \mathrm{~K}$ \\
$\sigma_{\max }$ & $50 \mathrm{nb}$ & $6 \mathrm{pb}$ \\
$I_{\text {RIB,ISOL }}$ & $\sim 10^{3} / \mathrm{s}$ & $3 \times 10^{6} / \mathrm{s}$ \\
$I_{\text {RIB,In-flight }}$ & $\sim 10^{5} / \mathrm{s}$ & $\sim 10^{6} / \mathrm{s}$ \\
$t_{1-\text { event,ISOL }}$ & $\sim 300$ years & $\sim 600$ years \\
$t_{1-\text { event,In-flight }}$ & $\sim 3$ years & $\sim 600$ years \\
\hline
\end{tabular}


nucleus of ${ }^{266} \mathrm{Rf}$ or ${ }^{291} \mathrm{Mc}$, with presently available intensities of ${ }^{22} \mathrm{O}$ and ${ }^{47} \mathrm{~K}$ and taking the theoretical cross-sections from Figs. 42, 43 and targets of $1 \mathrm{mg} / \mathrm{cm}^{2}$ thickness. The values in Table 3 indicate some trends which are confirmed if one looks to further example reactions. We want to stress that the numbers which we gave above do not yet take into account the intensity losses of the RIBs during acceleration (or deceleration, respectively) to Coulomb barrier energies.

The production yields of superheavy nuclei around the expected "island of stability" appear to be out of feasibility over the long term. But the synthesis of "light" superheavy nuclei with RIBs seems indeed realistic, particularly at future RIB facilities which will provide much higher beam intensities. By trend, inflight facilities seem best suited for such experiments because the intensities of the needed very light RIBs (roughly with $A<25$ ) are much higher in In-flight production compared to ISOL. We want to stress that there is other work which considered this topic and arrived at very similar conclusions (e.g., [367,368]).

\subsubsection{An "emergency solution”?}

The question arises if there is an "emergency solution" to learn about possible new shell closures in the region of superheavy elements. It can be answered with "maybe". Namely, by studying QF and FF reactions, which have significantly larger cross-sections than fusion residues, a possible influence of shell closures could be explored. The presumption is that shell closures of the compound system might also be reavealed in the mass, angle and energy distributions of QF and FF fragments. So far, the only appropriate facility to perform such experiments is HIE-ISOLDE at CERN, which can provide since recently RIBs with energies up to $10 \mathrm{MeV} /$ nucleon. Concerning the choice of projectile ion, one is also in such experiments bound to RIBs with intensities not lower than $\sim 10^{6} / \mathrm{s}$ in order to achieve statistical relevant data. This leaves ${ }^{95} \mathrm{Rb}$ as the most suitable projectile. In combination with ${ }^{209} \mathrm{Bi}$ targets, ${ }^{95} \mathrm{Rb}$ allows one to reach a compound system with proton and neutron shells at $Z=120, N=184$. An approved proposal for this experiment at HIE-ISOLDE is existing [369].

\subsection{Existing and upcoming RIB facilities}

There are activities in accelerator laboratories worldwide to upgrade existing RIB facilities or construct new ones to deliver high intensity exotic ion beams. The chart [370] in Fig. 44 gives an overview on presently existing ISOL and In-flight facilities as well as on facilities which are in planning or construction. In the following, we will pick out and describe a few of them to create an impression about the bandwidth of different technical approaches and potentials for future experiments, specifically in context with DIC at Coulomb barrier energies.

The "mother" of ISOL-type facilities is CERN ISOLDE which is in operation since 1967. Presently, ISOLDE is the only RIB facility which can provide intense exotic ion beams at Coulomb barrier energies up to $10 \mathrm{MeV} /$ nucleon by using the post-accelerators REX-ISOLDE [348] and HIE ISOLDE [349] (Fig. 45). These energies enable nuclear reactions also in very heavy collision systems. Typical for ISOL facilities, the highest yields are obtained for heavier alkali metals like $\mathrm{Rb}$ or $\mathrm{Cs}$, and noble gases like $\mathrm{Xe}$, for a broad variety of their isotopes from neutron-deficient to neutron-rich. With presently available beam intensities and high-quality ISOLtype beams, ISOLDE provides a good potential for studying DIC and MNT reactions with exotic projectiles (see also Sect. 5.3.2).
Fig. 44 Present and upcoming radioactive ion beam facilities worldwide [370]

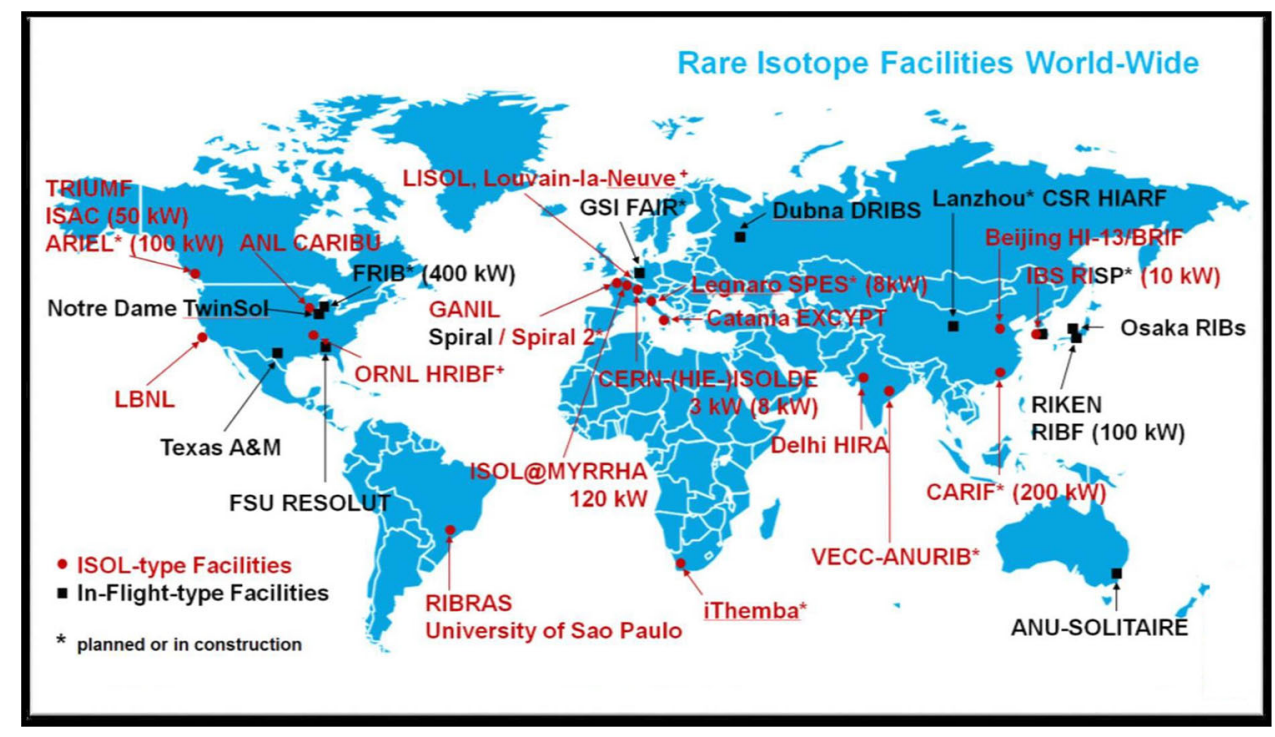




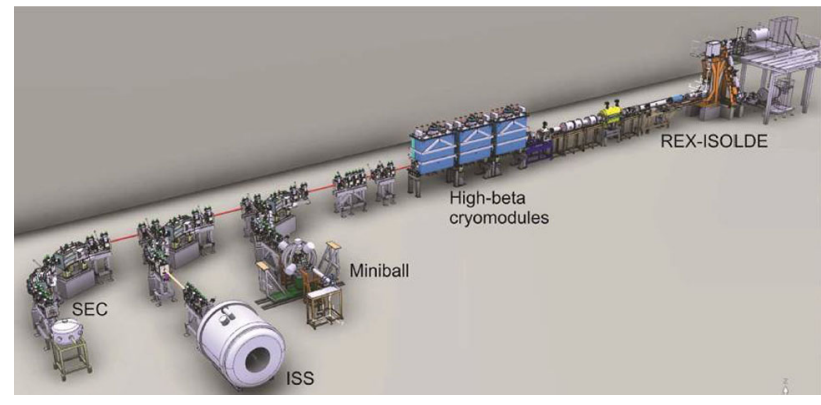

Fig. $453 D$ view [371] of the post-accelerators REX-ISOLDE and HIE-ISOLDE which can accelerate the ISOL-type RIBS of ISOLDE at CERN to energies up to $10 \mathrm{MeV} /$ nucleon. The picture shows also the three experiment stations

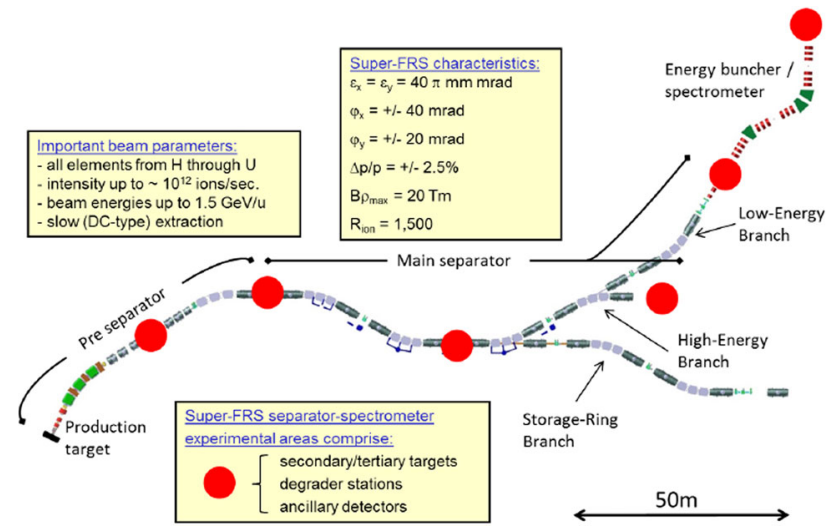

Fig. 46 Sketch of the future SuperFRS facility at FAIR [373] with its three experiment branches. The low-energy branch with the energy buncher spectrometer at its end is also foreseen to provide RIBs at Coulomb barrier energies for DIC studies. It is planned to decelerate the relativistic RIBs by using degraders

A typical representative of an In-flight facility is the fragment separator (FRS) facility [372] at GSI/FAIR which is in operation since 30 years and uses primary beams with energies up to $1 \mathrm{GeV} /$ nucleon. About 300 new isotopes were discovered at FRS so far. Presently, the new-generation RIB facility SuperFRS is under construction which is foreseen to deliver up to 100 times higher beam intensities compared to the present facility. Three branches for experiments are planned at the exit of the SuperFRS (see Fig. 46). The lowenergy branch, which will include an energy buncher, can also be used for DIC experiments at Coulomb barrier energies. At SuperFRS it is considered to decelerate the beams by using degraders. This leads, however, to fairly bad beam quality like discussed in Sect. 5.2.2. But there might be a way out which was so far not studied more closely, namely, to use a storage ring for RIB deceleration. For such studies, even the present FRS which is connected to the experimental storage ring (ESR) would be predestined.

The first powerful new-generation In-flight facility which is already in operation, is the Radioactive Isotope Beam Factory RIBF [374] at RIKEN Nishina Centre, Japan. Presently

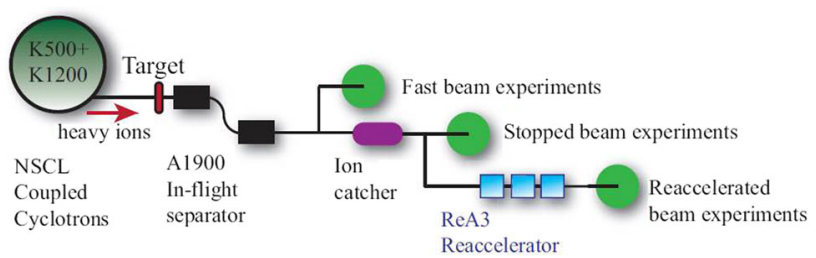

Fig. 47 Scheme of the radioactive ion beam facility at the National Superconducting Cyclotron Laboratory (NSCL) of Michigan State University [354]. After separation, the RIBs can either be used at full energy or stopped in an ion catcher. After extraction from the ion catcher one can use the beam directly for experiments at very low energy or reaccelerate it to Coulomb barrier energies

it uses primary beams up to uranium with maximum energies of about $350 \mathrm{MeV} /$ nucleon. Like the future SuperFRS, also RIBFs isotope separator BigRIPS will use superconducting magnets. RIBF is presently the worlds most powerful In-flight facility and provides the highest primary beam intensities.

The Facility for Rare Isotope Beams FRIB is also an Inflight type facility which is under construction at the National Superconducting Cyclotron Laboratory (NSCL) of Michigan State University [354]. There will be three branches for experiments at FRIB (Fig. 47). After In-flight separation, the RIBs can either be used at full energy at the "fast beam" branch. Or they can be stopped in an ion catcher. After extraction from the ion catcher, the beam can directly be used for experiments at very low energy. Or it can be reaccelerated to Coulomb barrier energies to use it for MNT experiments and DIC studies. As mentioned already in Sect. 5.2.2, it is planned to replace in the future the gas-stopping cell by a gas-filled reverse-cyclotron for beam deceleration.

Finally, there are RIB facilities under construction which will offer both, ISOL-type as well as In-flight production of exotic ion beams. One such representative is SPIRAL2 [375] at GANIL in Caen, France.

\section{The quest for new detection techniques}

Heavy MNT and fusion-evaporation products are emitted with low energies, therefore their identification via the universal $E-\Delta E$-TOF method fails due to the pulse-height deficit. For these nuclei, the most sensitive and effective identification technique is $\alpha$ decay tagging. The signatures of an $\alpha$ decay chain are mostly so clear that a single decay chain is sufficient to pin down the isotope. But it is obvious that the method is not universal and restricts to $\alpha$ emitters with appropriate half-lives and unambiguous decay chains. For example, it is not applicable for neutron-rich $\beta$ emitters below $\mathrm{Pb}$ if one considers to produce them in MNT reactions. All respective experiments used so far the emitted $\gamma$ rays for identification which is not nearly as sensitive as $\alpha$ 
spectroscopy; cross-sections below $1 \mu \mathrm{b}$ were not reached in any of these experiments. Also, many superheavy nuclei toward the neutron-rich side cannot be identified by $\alpha$ decays. Therefore, the success of extending the upper part of the nuclide chart depends crucially on the availability of sensitive and preferably universal detection techniques, regarding the expected small cross-sections. In the following we will consider possible techniques which are presently in discussion.

\subsection{High-precision mass measurements}

Reaction products can be identified if their mass is measured with sufficiently high resolution to distinguish different isobars. For this, mass resolving powers of $m / \Delta m \approx$ $\left(10^{5}-10^{6}\right)$ are needed like shown in the example in Fig. 48. Penning traps or multiple reflection time-of-flight mass spectrometers (MR-TOF-MS) are appropriate devices to reach these resolutions. In MNT reactions a broad region of isotopes is populated which results usually in the appearance of several isobars for each $A$, containing also already known ones. Therefore, the mass parabola will comprise also known masses beside the new ones and the location of the new mass on the parabola will identify the nucleus, even if $Z$ is not directly determined. Investigations of such a method are ongoing at the GSI fragment separator facility. The setup consists of a cryogenic stopping cell [376] and an MR-TOFMS [377] which are installed behind the fragment separator. The idea is to install targets with thicknesses of several $10 \mu \mathrm{m}$ inside the stopping cell which is filled with helium gas to stop the reaction products. This allows one to collect reaction products emitted to a wide angular range, which is particularly important for MNT products. The relativistic beams from FRS are decelerated by degraders, such that its mean energy is about $10 \mathrm{MeV} /$ nucleon when it impinges the reaction target inside the ion catcher. The stopped reac-

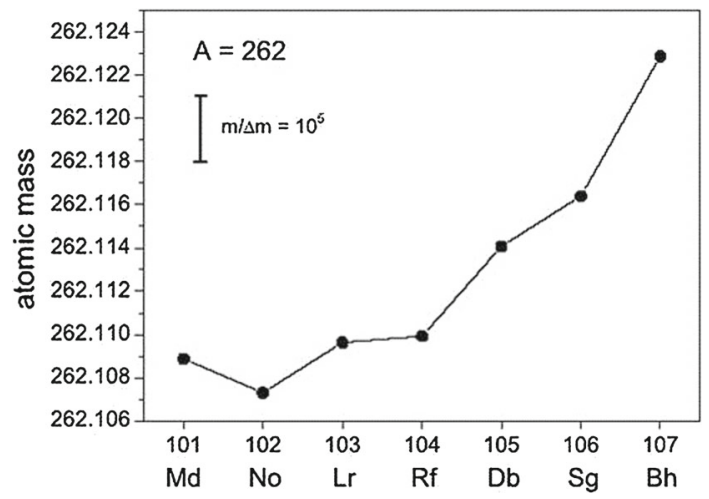

Fig. 48 Mass parabola for $A=260$ isotopes of elements with $Z=$ $101-107$. Masses are denoted up to the third decimal place. The figure reflects that some of the isobars can already be distinguished if their masses are measured with a resolution of $m / \Delta m=10^{5}$ tion products are extracted and injected to the MR-TOF-MS which allows for the broadband detection of different isotopes. For a sufficiently precise mass measurement, about 10 events are necessary. The method allows one to detect nuclei with half-lives $\geq 10 \mathrm{~ms}$. In order to avoid space charge effects in the stopping cell, the maximum beam intensity is limited. Estimates for uranium beams arrived at maximum intensities of $10^{7}$ ions/s. According to simulations, the method requires presently reaction product cross-sections of $100 \mu \mathrm{b}$ or more [378].

\subsection{Laser resonance ionization}

Another approach is the $Z$ identification of MNT products by laser resonance ionization. A respective system which focuses on neutron-rich beta-emitters below $\mathrm{Pb}$ was set up at the KEK Isotope Separation System (KISS) at RIKEN [379, 380]. The MNT products are stopped in an argon gas filled ion catcher. The ion catcher is doughnut-shaped to prevent the primary beam from entering the cell. Laser resonance ionization inside the ion catcher is used for $Z$ selection. After extraction from the gas cell, the ions are passing a magnetic dipole field for $A / q$ separation. The selected ions can then be guided to a detection system to perform spectroscopy studies. The method is applicable for ions with lifetime of $1 \mathrm{~s}$ or more. This is mainly determined by the extraction times from the gas catcher which are about $0.5 \mathrm{~s}$. The overall efficiency of the present KISS setup is on the scale of $0.1 \%$.

\subsection{Combination of mass separation and decay tagging}

A combination of mass separation and decay tagging in the region of heaviest elements was for the first time successfully applied at LBNL (see [381] and the references therein). Isotopes of element 115 were produced in fusion reactions of ${ }^{48} \mathrm{Ca}+{ }^{243} \mathrm{Am}$. After passing the gas-filled magnetic separator BGS, they were stopped in a radiofrequency gas catcher. After extraction, ion bunches were created in an RFQ trap and from there injected in a trochoid spectrometer. The separated ions were then implanted in a silicon strip detector, where their $\alpha$ decay chains were measured. With this method it was possible to measure directly the $A$ of ${ }^{288} \mathrm{Mc}$ and its decay daughter ${ }^{284} \mathrm{Nh}$. Ion losses occur mainly during stopping and extraction from the gas catcher. The transport efficiency from the exit of BGS to the focal plane detector is denoted in [381] with $14 \%$. With this, the method is applicable for production cross-sections on the picobarn scale. Transportation times through the system are on the scale of several $10 \mathrm{~ms}$.

\section{Summary considerations}

The present chart of nuclides reveals still large empty areas which need to be filled. To our opinion, the "conventional" 
fragmentation, fusion and fission reactions will continue to provide the highest potential for laboratory nucleosynthesis. Most promising are the new powerful exotic ion beam facilities which are presently emerging in many places. They are designed to deliver up to three orders of magnitude higher beam currents which will allow one to extend considerably the chart of nuclides in the region below uranium by further using fragmentation and fission reactions. Particularly the sill sparse area of neutron-rich r-process nuclei will profit from it.

More critical is the region of transuranium nuclei which is presently populated solely in complete-fusion reactions. Particularly their limitation toward the neutron-rich side cannot be overcome readily because fusion reactions with stable projectiles lead to rather neutron-deficient residues due to the bending of the stability line. Some new isotopes can be produced in charged particle evaporation channels in the de-excitation of $\mathrm{CN}$ [382-385]. Fusion reactions with RIBs would be the most elegant way to produce neutron-rich transuranium nuclei, but the available intensities of suitable RIBs are still far too low and would mostly require unrealistically long measurement times. Regarding this situation, also the new generation of RIB facilities will not provide a satisfactory solution. Interesting is that about 50 new superheavy isotopes between $Z=(104-118)$ can still be produced in fusion reactions with stable projectiles and actinide targets and would fill the present gap between the regions of cold and hot fusion-evaporation residues.

Aiming for a solution to synthesize more neutron-rich superheavy nuclei, we arrive at MNT reactions. The kinematics of MNT reactions is less favorable than fusion kinematics because it leads to broad angular and energy distributions of the residual nuclei. This makes their efficient separation from background events difficult and leads necessarily to larger losses of reaction products compared to fusion reactions. Nevertheless, the so far accomplished experiments and theoretical predictions indicate that one can expect a variety of new isotopes from MNT reactions. Toward the neutronrich side, one can realistically expect new isotopes of elements with proton numbers $Z \leq 108$ and neutron numbers next to the already known ones. This will allow for the direct synthesis of the endpoint nuclei of hot fusion decay chains. Rather unrealistic seems from the present point of view the synthesis of very heavy MNT products with $Z>108$. A large potential of MNT reactions we expect, however, for the region of very neutron-deficient transuranium nuclei, even if it can also be populated in complete-fusion reactions with stable projectiles. Experiments revealed so far that the yields which can be expected from complete fusion and MNT are comparable in this area. However, in MNT reactions a wide span of different nuclides can be populated at the same experimental setting due to their broad excitation functions which makes MNT reactions in this case more efficient.
There remains the question how to reach the predicted center of the "island of stability" at $Z=114,120-126$ and $N=184$. Nuclei with such large neutron numbers are from present state of knowledge neither reachable in MNT reactions nor in complete-fusion reactions with RIBs due to the tiny cross-sections. But RIBs might hold an "emergency solution". The RIB intensities are large enough to study quasifission and fusion-fission reactions in respective systems because these reactions have large cross-sections. The hope is that possible shell closures leave their fingerprint not only in fusion-evaporation residues but also in the mass, angle and energy distributions of quasi-fission and fusion-fission fragments. The challenge is to distinguish these signatures in the measured distributions. We will start respective studies in near future at the new HIE-ISOLDE facility at CERN.

Synthesis of new nuclides is usually connected with experiments at the limits of feasibility. Therefore, reliable model predictions are extremely important for the successful conductance of the experiments. Roughly, the different models can be divided into macroscopic, macroscopic-microscopic, and purely microscopic. Among them, the presently most wide-spread models are the macroscopic-microscopic models which can again be divided in two main branches. One is the class of DNS models, which use the diffusion master equation, diabatic internuclear potentials and the quantum nature of interacting nuclei. Models of the other class use Langevin-type equations of motion and almost adiabatic internuclear potentials. These models indicate some common trends and act as good guidelines for experiments.

\section{Ten supplementary questions}

1. How many new transuranium and superheavy nuclei can we expect in the near and mid-term future?

We estimate that MNT reactions can provide roughly 150 new transuranium isotopes in the neutron-deficient and neutron-rich area. Another 50 new superheavy nuclei can still be expected from fusion reactions with stable beams, resulting in a total of about 200 new isotopes. This is a good number compared to the 348 presently known transuranium nuclides.

2. Which collision systems seem more favorable to synthesize neutron-rich superheavy nuclei in MNT reactions, intermediate heavy projectiles on heavy target nuclei like ${ }^{48} \mathrm{Ca}+{ }^{248} \mathrm{Cm}$, or heavy projectiles on heavy target nuclei like ${ }^{238} \mathrm{U}+{ }^{248} \mathrm{Cm}$ ?

Experimental results reveal that the choice of projectile plays no big role as long as the envisaged MNT products are not too far from the target nucleus (see Sect. 4.2.2). For MNT products with up to 5 protons above the target, the crosssections obtained with ${ }^{48} \mathrm{Ca}$ and ${ }^{238} \mathrm{U}$ projectiles on ${ }^{248} \mathrm{Cm}$ 
targets were the same within error bars. But one must also consider that lighter beams like ${ }^{48} \mathrm{Ca}$ are usually provided with much higher intensities than ${ }^{238} \mathrm{U}$ beams, which will lead to, respectively, larger yields at the same cross-section.

3. Which are the most serious bottlenecks if we plan to use MNT reactions to produce neutron-rich superheavy nuclei?

We regard the lack of appropriate separation and detection techniques as most serious restriction. The wide angular and energy distributions of MNT products make In-flight separation inefficient and for isotope identification the sensitive $\alpha$ decay tagging method largely fails in the neutron-rich superheavy region. We have to deal with cross-sections on the picobarn scale resulting in event rates of one nucleus per week. Therefore we cannot afford large losses, and the sensitivity of the experimental setup must approximately meet the production cross-section. The most sensitive techniques which are presently investigated as possible alternatives to $\alpha$ decay tagging are so far applicable for microbarn crosssections and we still need to bridge six orders of magnitude to make them suitable for picobarns.

4. Are $M N T$ reactions really better than fragmentation to synthesize $N=126$ nuclei?

At the moment, we regard fragmentation reactions still as unrivalled to produce neutron-rich nuclei below $\mathrm{Pb}$. It is true that first experiments confirmed model predictions according to which MNT cross-sections are comparable to, or even exceeding fragmentation cross-sections. But in projectile fragmentation reactions the experimental conditions are unequally better which means that: (i) 10000 times thicker targets lead to 10,000 times higher yields for the same crosssection and beam intensity, (ii) strongly forward directed kinematics enables very efficient In-flight separation, (iii) relativistic energies allow for isotope identification by $\mathrm{E}-\Delta \mathrm{E}-$ TOF measurement, sensitive enough to detect single isotopes independent of their decay properties. MNT products in this region can so far only be identified via their gamma decays which means serious restrictions: the method is only applicable if excited states in the daughter nucleus are populated with sufficient branching and gamma transitions in the daughter nucleus must be already well known in order to attribute them correctly. Nevertheless, it is still very important to collect more data on MNT reactions in this region. Particularly the observed trend that MNT cross-sections increase strongly with respect to fragmentation cross-sections toward lower $\mathrm{Z}$ of the MNT products is very interesting. In reactions of $\mathrm{Xe}+\mathrm{Pb}, \mathrm{MNT}$ cross-sections started to exceed fragmentation cross-sections by four orders of magnitude for residues with $\mathrm{N}=126, \mathrm{Z} \leq 77$. So far, these MNT cross-sections were deduced indirectly from the ones of projectile-like MNT products and it is now very important to verify this effect by directly identifying the target-like MNT products.
5. Are MNT reactions with RIBs an option to produce neutron-rich isotopes?

It is revealed experimentally and theoretically that more neutrons in the system shift the isotopic distributions of MNT products toward the neutron-rich side. Therefore, MNT reactions with neutron-rich RIBs should lead to more neutronrich MNT products. However, one is quite restricted in the choice of suitable exotic projectile nuclei from terms of intensity. The best suitable RIBs are heavy alkali elements like Rb or Cs and noble gases like Xe. They are available with good intensity for a broad region of their exotic isotopes. But the intensities of stable and relatively neutron-rich beams like ${ }^{48} \mathrm{Ca}$ are still some orders of magnitude larger. Finally, it is the product of beam intensity and cross-section, $I_{\text {beam }} \times \sigma$, which determines if the yield of a certain isotope is larger with an exotic or with a stable projectile.

6. Would it be better to use inverse kinematics for MNT reactions?

Apart from the advantages of the more forward focused kinematics in such reactions, one has to consider some technical aspects. For example, very heavy beams are usually delivered with much smaller intensities than lighter beams which leads to smaller yields at a given cross-section. Also, if one uses the heavy reaction partner as beam, one is limited to uranium as heaviest possible projectile. This is, for example, unfavorable if one wants to synthesize new superheavy nuclei which are well above uranium. In earlier experiments it was found that the cross-section of heavy MNT products decreases on average by one order of magnitude with the transfer of every proton more.

7. Which still empty region on the nuclide chart is most difficult to access?

This is the predicted island of stability in the superheavy element region. Fusion reactions with stable projectiles do not provide enough neutrons. Fusion reactions with RIBs fail due to the by far too small RIB intensities. Transfer reactions appear not feasible due to the tiny cross-sections. In near and mid-term future one can expect only indirect information about possible enhanced stability in this area, for example by studying quasi-fission and fusion-fission reactions with neutron-rich RIBs (see Sect. 5.3.2).

8. Are MNT reactions rather an emergency solution or a rich source for new isotopes?

It depends on the region which we intend to populate. To produce new neutron-rich superheavy nuclei, MNT reactions are presently the only alternative - and to our opinion an emergency solution. The kinematically much more favorable fusion reactions are not applicable here due to the lack of neutrons in the system if one uses stable projectiles and due to the tiny intensities if one uses neutron-rich RIBs. The weak point in low-energy MNT reactions is their kinematics. Large 
Table 4 Overview of techniques, elemental region of MNT products and smallest cross-sections which were observed in previous and present experiments on MNT reactions

\begin{tabular}{lll}
\hline Technique & MNT products & Sensitivity \\
\hline$B \rho-\Delta E-E$ & $Z=6-17$ & $\mu \mathrm{b}$ \\
TOF- $\Delta E-E$ & $Z=20-30$ & $\mu \mathrm{b}$ \\
$B \rho+$ decay tagging & $\mathrm{Z} \leq 89$ & $\mu \mathrm{b}$ \\
Chemical separation + decay tagging & $\mathrm{Z} \leq 103$ & $20 \mathrm{nb}$ \\
Velocity filter + decay tagging & $\mathrm{Z} \leq 102$ & $1 \mathrm{nb}$ \\
\hline
\end{tabular}

angular distributions and energy spread makes their separation and distinction from background events very difficult.

To produce neutron-rich $N=126$ nuclei below $\mathrm{Pb}, \mathrm{MNT}$ reactions might even be not necessary because the very effective fragmentation reactions seem still the better alternative (see question 4).

Finally, there is the region of neutron-deficient transuranium isotopes which can principally also be accessed in complete-fusion reactions. Experimental data reveal that the yields become increasingly comparable in fusion and MNT reactions the more one moves toward the neutron-deficient side. At that point, an advantage of MNT reactions comes into play, namely, that they are not selective on few distinct reaction products like complete fusion but allow for the population of a broad region of nuclei in the same experiment. Here, indeed MNT reactions might be the better source for new isotopes.

\section{Which are the best experimental setups for nucleosynthesis in MNT reactions?}

Table 4 summarizes the experimental techniques which were so far applied in searches for new isotopes in MNT reactions. The lowest cross-sections were so far reached with the radiochemical and the velocity filter method. In some sense they are complementary to each other. With radiochemical methods MNT products emitted to large opening angles can be collected while the velocity filter is selective on products emitted to forward angles. Another difference is that radiochemical methods need more time and are better suitable for nuclei with minimum half-lives of about $1 \mathrm{~min}$. The In-flight technique is suitable for very short-lived nuclides, where the limitation is mainly given by the flight time of the nuclei through the separator which is typically on the $1 \mu \mathrm{s}$ scale. An important and noteworthy point is that both techniques reached their small cross-section limits only for isotopes which can be identified by $\alpha$ decay tagging!

10. Is it reasonable to consider MNT reactions at intermediate energies?

Binary reaction products have been experimentally observed among the products of projectile fragmentation reactions at incident energies around and above the Fermi energy. The yields of the most neutron-rich isotopes of light nuclei tend toward the $\mathrm{Q}_{g g}$ systematics, indicating MNT processes. Therefore, the question arises of how important the
MNT binary process is in peripheral collisions at quite high bombarding energies. Experimental and theoretical investigations of this subject are highly desirable.

Acknowledgements Open Access funding provided by Projekt DEAL. This work was partly supported by RFBR (17-52-12015) and DFG Grants HE 5469/3-1. The work of N.V.A. was supported by Tomsk Polytechnic University Competitiveness Enhancement Program Grant A.D-T was supported by the STFC Consolidated Grant (ST/P005314/1). We would like to express our gratitude to Aradhana Shrivastava for providing Fig. 35.

Data Availability Statement This manuscript has no associated data or the data will not be deposited. [Authors' comment: Any data that support the findings of this study are included within the article.]

Open Access This article is licensed under a Creative Commons Attribution 4.0 International License, which permits use, sharing, adaptation, distribution and reproduction in any medium or format, as long as you give appropriate credit to the original author(s) and the source, provide a link to the Creative Commons licence, and indicate if changes were made. The images or other third party material in this article are included in the article's Creative Commons licence, unless indicated otherwise in a credit line to the material. If material is not included in the article's Creative Commons licence and your intended use is not permitted by statutory regulation or exceeds the permitted use, you will need to obtain permission directly from the copyright holder. To view a copy of this licence, visit http://creativecomm ons.org/licenses/by/4.0/.

\section{References}

1. G. Fea, Nuovo Cimento 6, 1 (1935)

2. M. Thoennessen, Rep. Prog. Phys. 76, 056301 (2013)

3. M. Thoennessen, The Discovery of Isotopes, A Complete Compilation (Springer International Publishing, Cham, 2016)

4. O. Hahn, F. Strassmann, Naturwiss 27, 11 (1939)

5. O. Hahn, F. Strassmann, Naturwiss 27, 89 (1939)

6. H.H. Hopkins Jr., B.B. Cunningham, Phys. Rev. 73, 1406 (1948)

7. J. Wilczynski, V.V. Volkov, P. Decowski, Yad. Fiz. 5, 942 (1967)

8. A.G. Artukh, G.F. Gridnev, V.L. Mikheev, V.V. Volkov, Nucl. Phys. A 137, 348 (1969)

9. G.F. Gridnev, V.V. Volkov, J. Wilczynski, Nucl. Phys. A 142, 385 (1970)

10. A.G. Artukh, V.V. Avdeichikov, G.F. Gridnev, V.L. Mikheev, V.V. Volkov, J. Wilczynski, Nucl. Phys. A 176, 284 (1971)

11. A.G. Artukh, G.F. Gridnev, V.L. Mikheev, V.V. Volkov, J. Wilczynski, Nucl. Phys. A 211, 299 (1973)

12. A.G. Artukh, J. Wilczynski, V.V. Volkov, G.F. Gridnev, V.L. Mikhiev, Yad. Fiz. 17, 1126 (1973)

13. A.G. Artukh, G.F. Gridnev, V.L. Mikheev, V.V. Volkov, J. Wilczynski, Nucl. Phys. A 215, 91 (1973)

14. J. Galin, D. Guerreau, M. Lefort, J. Peter, X. Tarrago, R. Basile, Nucl. Phys. A 159, 461 (1970) 
15. F. Hanappe, M. Lefort, C. Ngo, J. Peter, B. Tamain, Phys. Rev. Lett. 32, 738 (1974)

16. L.G. Moretto, D. Heunemann, R.C. Jared, R.C. Gatti, S.G. Thompson, Physics and Chemistry of Fission 1973, vol. 2 (IAEA, Vienna, 1974), p. 351

17. K.L. Wolf, J.P. Unik, J.R. Huizenga, J. Birkelund, H. Freiesleben, V.E. Viola, Phys. Rev. Lett. 33, 1105 (1974)

18. S.G. Thompson, L.G. Moretto, R.C. Jared, R.P. Babinet, J. Galin, M.M. Fowler, R.C. Gatti, J.B. Hunter, Phys. Scr. Suppl. A 10, 36 (1974)

19. C.H. Dasso, G. Pollarolo, A. Winther, Phys. Rev. Lett. 73, 1907 (1994)

20. G.G. Adamian, N.V. Antonenko, W. Scheid, Phys. Rev. C 68, 034601 (2003)

21. G.G. Adamian, N.V. Antonenko, Phys. Rev. C 72, 064617 (2005)

22. G.G. Adamian, N.V. Antonenko, A.S. Zubov, Phys. Rev. C 71, 034603 (2005)

23. YuE Penionzhkevich, G.G. Adamian, N.V. Antonenko, Phys. Lett. B 621, 119 (2005)

24. YuE Penionzhkevich, G.G. Adamian, N.V. Antonenko, Eur. Phys. J. A 27, 187 (2006)

25. G.G. Adamian, N.V. Antonenko, S.M. Lukyanov, YuE Penionzhkevich, Phys. Rev. C 78, 024613 (2008)

26. V.I. Zagrebaev, W. Greiner, Phys. Rev. Lett. 101, 122701 (2008)

27. Z.-Q. Feng, G.-H. Jin, J.-Q. Li, Phys. Rev. C 80, 068601 (2009)

28. G.G. Adamian, N.V. Antonenko, V.V. Sargsyan, W. Scheid, Phys. Rev. C 81, 057602 (2010)

29. G.G. Adamian, N.V. Antonenko, V.V. Sargsyan, W. Scheid, Phys. Rev. C 81, 024604 (2010)

30. D.J. Kedziora, C. Simenel, Phys. Rev. C 81, 044613 (2010)

31. V.I. Zagrebaev, Walter Greiner, Phys. Rev. C 83, 044618 (2011)

32. K. Sekizawa, K. Yabana, Phys. Rev. C 88, 014614 (2013)

33. V.I. Zagrebaev, W. Greiner, Phys. Rev. C 87, 034608 (2013)

34. V.I. Zagrebaev, B. Fornal, S. Leoni, Walter Greiner, Phys. Rev. C 89, 054608 (2014)

35. Myeong-Hwan Mun, G.G. Adamian, N.V. Antonenko, Yongseok Oh, Youngman Kim, Phys. Rev. C 89, 034622 (2014)

36. K. Zhao, Z. Li, N. Wang, Y. Zhang, Q. Li, Y. Wang, X. Wu, Phys. Rev. C 92, 024613 (2015)

37. K. Zhao, Z. Li, Y. Zhang, N. Wang, Q. Li, C. Shen, Y. Wang, X. Wu, Phys. Rev. C 94, 024601 (2016)

38. L. Zhu, J. Su, W.-J. Xie, F.-S. Zhang, Phys. Rev. C 94, 054606 (2016)

39. K. Sekizawa, K. Yabana, Phys. Rev. C 93, 054616 (2016)

40. C. Li, F. Zhang, J. Li, L. Zhu, J. Tian, N. Wang, F.-S. Zhang Phys. Rev. C 93, 014618 (2016)

41. A.V. Karpov, V.V. Saiko, Phys. Rev. C 96, 024618 (2017)

42. L. Zhu, F.-S. Zhang, P.-W. Wen, J. Su, W.-J. Xie, Phys. Rev. C 96, 024606 (2017)

43. K. Sekizawa, Phys. Rev. C 96, 014615 (2017)

44. K. Sekizawa, Phys. Rev. C 96, 041601 (2017)

45. Z.-Q. Feng, Phys. Rev. C 95, 024615 (2017)

46. F. Niu, P.-H. Chen, Y.-F. Guo, G.-W. Ma, Z.-Q. Feng, Phys. Rev. C 96, 064622 (2017)

47. F. Niu, P.-H. Chen, Y.-F. Guo, G.-W. Ma, Z.-Q. Feng, Phys. Rev. C 97, 034609 (2018)

48. L. Zhu, P.-W. Wen, C.-J. Lin, X.-J. Bao, J. Su, C. Li, C.-C. Guo, Phys. Rev. C 97, 044614 (2018)

49. K. Sekizawa, Front. Phys. 7, 20 (2019)

50. Long Zhu, Cheng Li, Su Jun, Chen-Chen Guo, Wei Hua, Phys. Lett. B 791, 20 (2019)

51. V.V. Saiko, A.V. Karpov, Phys. Rev. C 99, 014613 (2019)

52. V.V. Saiko, A.V. Karpov, Acta Phys. Pol. B 50, 495 (2019)

53. Z. Wu, L. Guo, Phys. Rev. C 100, 014612 (2019)

54. S. Ayik et al., Phys. Rev. C 100, 014609 (2019)
55. Myeong-Hwan Mun, Kyujin Kwak, G.G. Adamian, N.V. Antonenko, Phys. Rev. C 99, 054627 (2019)

56. E. Haseltine, http://discovermagazine.com/2002/feb/cover

57. V.V. Volkov, Phys. Rep. 44, 93 (1978)

58. R. Bass, Nuclear Reactions with Heavy Ions (Springer, Berlin, 1980), p. 203

59. W. Nörenberg, Heavy Ion Collisions, ed. by R. Bock, vol. 2 (NorthHolland, Amsterdam, 1980), p. 1

60. R.V. Jolos, R. Schmidt, Fiz. Elem. Chastits At. Yadra 12, 324 (1981)

61. R.V. Jolos, R. Schmidt, Sov. J. Part. Nucl. 12, 129 (1981)

62. V.V. Volkov, Nuclear Reactions of Deep Inelastic Transfers (Energoizdat, Moscow, 1982)

63. V.V. Volkov, Treatise on Heavy-Ion Science, ed. by D.A. Bromley, vol. 8 (Plenum Press, New York, 1989) p. 101

64. W.U. Schröder, J.R. Huizenga, Treatise on Heavy-Ion Science, ed. by D.A. Bromley, vol. 8 (Plenum Press, New York, 1989), p. 115

65. J. Wilczynski, Phys. Lett. B 47, 484 (1973)

66. H.H. Freiesleben, J.V. Kratz, Phys. Rep. 106, 1 (1984)

67. R.T. de Souza, J.R. Huizenga, W.U. Schröder, Phys. Rev. C 37, 1901 (1988)

68. R.T. de Souza, W.U. Schröder, J.R. Huizenga, J. Tõke, S.S. Datta, J.L. Wile, Phys. Rev. C 39, 114 (1989)

69. R. Planeta, S.H. Zhou, K. Kwiatkowski, W.G. Wilson, V.E. Viola, H. Breuer, D. Benton, F. Khazaie, R.J. McDonald, A.C. Mignerey, A. Weston-Dawkes, R.T. de Souza, J.R. Huizenga, W.U. Schröder, Phys. Rev. C 38, 195 (1988)

70. V.F. Comas, S. Heinz, S. Hofmann, D. Ackermann, J. Heredia, F.P. Heßberger, J. Khuyagbaatar, B. Kindler, B. Lommel, R. Mann, Eur. Phys. J. A 48, 180 (2012)

71. V.F. Comas, S. Heinz, S. Hofmann, D. Ackermann, J. Heredia, F.P. Heßberger, J. Khuyagbaatar, B. Kindler, B. Lommel, R. Mann, Eur. Phys. J. A 49, 112 (2013)

72. M. Wilpert, B. Gebauer, Th Wilpert, W. von Oertzen, H.G. Bohlen, J. Speer, Phys. Rev. C 51, 680 (1995)

73. S. Heinz, O. Beliuskina, V. Comas, H.M. Devaraja, C. Heinz, S. Hofmann, E. Kozulin, F. Morherr, G. Münzenberg, D. Ackermann, F.P. Heßberger, B. Kindler, B. Lommel, R. Mann, J. Maurer, Eur. Phys. J. A 51, 140 (2015)

74. H. Freiesleben, K.D. Hildenbrand, F. Pühlhofer, W.F.W. Schneider, R. Bock, Dv Harrach, H.J. Specht, Z. Phys. A 292, 171 (1979)

75. C. Golabek, S. Heinz, W. Mittig, F. Rejmund, A.C.C. Villari, S. Bhattacharyva, D. Boilley, G. De France, A. Drouart, L. Gaudefroy, L. Giot, V. Maslov, M. Morjean, G. Mukherjee, Yu. Penionzkevich, P. Roussel-Chomaz, C. Stodel, Eur. Phys. J. A 43, $251(2010)$

76. J. Töke, R. Bock, G.X. Dai, A. Gobbi, S. Gralla, K.D. Hildenbrand, J. Kuzminski, W.F.J. Müller, A. Olmi, H. Stelzer, B.B. Back, S. Bjørnholm, Nucl. Phys. A 440, 327 (1985)

77. E.M. Kozulin, A.A. Bogachev, M.G. Itkis, I.M. Itkis, G.N. Knyazheva, N.A. Kondratiev, L. Krupa, I.V. Pokrovsky, E.V. Prokhorova, Instr. Exp. Techn. 51, 44 (2008)

78. E.M. Kozulin, G.N. Knyazheva, I.M. Itkis, M.G. Itkis, A.A. Bogachev, E.V. Chernysheva, L. Krupa, F. Hanappe, O. Dorvaux, L. Stuttge, W.H. Trzaska, C. Schmitt, G. Chubarian, Phys. Rev. C 90, 054608 (2014)

79. E.M. Kozulin, G.N. Knyazheva, K.V. Novikov, I.M. Itkis, M.G. Itkis, S.N. Dmitriev, YuTs Oganessian, A.A. Bogachev, N.I. Kozulina, I. Harca, W.H. Trzaska, T.K. Ghosh, Phys. Rev. C 94, 054613 (2016)

80. M.G. Itkis, I.M. Itkis, G.N. Knyazheva, E.M. Kozulin, J. Phys. Conf. Ser. 863, 012043 (2017)

81. E.M. Kozulin, G.N. Knyazheva, T.K. Ghosh, A. Sen, I.M. Itkis, M.G. Itkis, K.V. Novikov, I.N. Diatlov, I.V. Pchelintsev, C. Bhattacharya, S. Bhattacharya, K. Banerjee, E.O. Saveleva, I.V. Vorobiev, Phys. Rev. C 99, 014616 (2019) 
82. K. Nishio, J. Phys. Conf. Ser. 282, 012011 (2011)

83. D.J. Hinde et al., EPJ Web Conf. 131, 04004 (2016)

84. E. Prasad, D.J. Hinde, E. Williams, M. Dasgupta, I.P. Carter, K.J. Cook, D.Y. Jeung, D.H. Luong, C.S. Palshetkar, D.C. Rafferty, K. Ramachandran, C. Simenel, A. Wakhle, Phys. Rev. C 96, 034608 (2017)

85. K. Hammerton, D.J. Morrissey, Z. Kohley, D.J. Hinde, M. Dasgupta, A. Wakhle, E. Williams, I.P. Carter, K.J. Cook, J. Greene, D.Y. Jeung, D.H. Luong, S.D. McNeil, C. Palshetkar, D.C. Rafferty, C. Simenel, K. Stiefel, Phys. Rev. C 99, 054621 (2019)

86. A. Türler et al., Phys. Rev. C 46, 1364 (1992)

87. R.V. Jolos, S.M. Lukyanov, A.K. Nasirov, V.P. Permyakov, V.S. Salamatin, G.G. Chubarian, Yad. Fiz. 50, 382 (1989)

88. R.V. Jolos, S.M. Lukyanov, A.K. Nasirov, V.P. Permyakov, V.S. Salamatin, G.G. Chubarian, Sov. J. Nucl. Phys. 50, 239 (1989)

89. W. Bohne, P. Fröbrich, K. Grabisch, K. Hartmann, H. Lehr, H. Morgenstern, W. Stöffler, Z. Phys. A 313, 19 (1983)

90. P. Gippner, K.D. Schilling, W. Seidel, F. Stary, E. Will, H. Sodan, S.M. Lukyanov, V.S. Salamatin, YuE Penionzhkevich, G.G. Chubarian, R. Schmidt, Z. Phys. A 325, 335 (1986)

91. G. Guarino, A. Gobbi, K.D. Hildenbrand, W.F.J. Müller, A. Olmi, H. Sann, S. Bjørnholm, G. Rudolf, Nucl. Phys. A 424, 157 (1984)

92. V.V. Volkov, Izv. Akad. Nauk SSSR Ser. Fiz 50, 1879 (1986)

93. K.D. Hildenbrand, H. Freiesleben, F. Pühlhofer, W.F.W. Schneider, R. Bock, Dv Harrach, H.J. Specht, Phys. Rev. Lett. 39, 1065 (1977)

94. T. Tanabe, R. Bock, M. Dakowski, A. Gobbi, H. Sann, H. Stelzer, U. Lynen, A. Olmi, D. Pelte, Nucl. Phys. A 342, 194 (1980)

95. K.D. Hildenbrand, H. Freiesleben, A. Gobbi, U. Lynen, A. Olmi, H. Sann, E.C. Wu, Nucl. Phys. A 405, 179 (1983)

96. A.B. Quint et al., Z. Phys. A 346, 119 (1993)

97. N.V. Antonenko, E.A. Cherepanov, A.K. Nasirov, V.P. Permjakov, V.V. Volkov, Phys. Lett. B 319, 425 (1993)

98. S. Agarwal, J. Galin, B. Gatty, D. Guerreau, M. Lefort, X. Tarrago, R. Babinet, J. Girard, Z. Phys. A 296, 287 (1980)

99. B.B. Back, R.R. Betts, J.E. Gindler, B.D. Wilkins, S. Saini, M.B. Tsang, C.K. Gelbke, W.G. Lynch, M.A. McMahan, P.A. Baisden, Phys. Rev. C 32, 195 (1985)

100. W.Q. Shen, J. Albinski, A. Gobbi, S. Gralla, K.D. Hildenbrand, N. Herrmann, J. Kuzminski, W.F.J. Müller, H. Stelzer, J. Töke, B.B. Back, S. Bjornholm, S.P. Sorensen, Phys. Rev. C 36, 115 (1987)

101. B.B. Back, S. Bjornholm, T. Dossing, W.Q. Shen, K.D. Hildenbrand, A. Gobbi, S.P. Sorensen, Phys. Rev. C 41, 1495 (1990)

102. H.Q. Zhang, C.L. Zhang, C.J. Lin, Z.H. Liu, F. Yang, A.K. Nasirov, G. Mandaglio, M. Manganaro, G. Giardina, Phys. Rev. C 81, 034611 (2010)

103. A. Shamlath et al., Phys. Rev. C 95, 034610 (2017)

104. S. Hofmann, G. Münzenberg, Rev. Mod. Phys. 72, 733 (2000)

105. YuTs Oganessian, V.K. Utyonkov, Rep. Prog. Phys 78, 036301 (2015)

106. J. Maruhn, W. Greiner, Z. Phys. 251, 431 (1972)

107. D.H.E. Gross, H. Kalinowski, Phys. Rep. 45, 175 (1978)

108. P. Fröbrich, Phys. Rep. 116, 337 (1984)

109. W.J. Swiatecki, Prog. Part. Nucl. Phys. 4, 383 (1980)

110. W.J. Swiatecki, Phys. Scr. 24, 113 (1981)

111. D.H.E. Gross, R.C. Nayak, L. Satpathy, Z. Phys. A 299, 63 (1981)

112. M. Münchow, W. Scheid, Nucl. Phys. A 468, 59 (1987)

113. J.A. Maruhn, W. Greiner, W. Scheid, Heavy Ion Collisions, ed. by R. Bock, vol. 2 (North-Holland, Amsterdam, 1980), p. 397

114. R. Schmidt, Fiz. Elem. Chastits At. Yadra 13, 1203 (1982)

115. R. Schmidt, Sov. J. Part. Nucl. 13, 513 (1982)

116. O.F. Nemets, V.G. Neudachin, A.T. Rudtchik, YuF Smirnov, YuM Tchuvilski, Nucleon Associations in Nuclei and Nuclear Reactions of Multi-Nucleon Transfers (Naukova Dumka, Kiev, 1988). (in Russian)
117. A. Lukasiak, W. Cassing, W. Nörenberg, Nucl. Phys. A 426, 181 (1984)

118. D. Berdichevsky, A. Lukasiak, W. Nörenberg, P. Rozmej, Nucl. Phys. A 499, 609 (1989)

119. J. Blocki, Y. Boneh, J.R. Nix, J. Randrup, M. Robel, A.J. Sierk, W.J. Swiatecki, Ann. Phys. (N.Y.) 113, 330 (1978)

120. H.J. Fink, J. Maruhn, W. Scheid, W. Greiner, Z. Phys. A 268, 321 (1974)

121. A. Pop, A. Sandulescu, H. Scutaru, W. Greiner, Z. Phys. A 329, 357 (1988)

122. W. Nörenberg, Phys. Lett. B 52, 289 (1974)

123. L.G. Moretto, J.S. Sventek, Phys. Lett. B 58, 26 (1975)

124. S. Ayik, B. Schürmann, W. Nörenberg, Z. Phys. A 277, 299 (1976)

125. S. Ayik, B. Schürmann, W. Nörenberg, Z. Phys. A 279, 145 (1976)

126. H. Hofmann, Phys. Rep. 284, 137 (1997)

127. H.A. Weidenmüller, Progr. Part. Nucl. Phys. 3, 49 (1980)

128. J. Randrup, Nucl. Phys. A 307, 319 (1978)

129. H. Feldmeier, Rep. Prog. Phys. 50, 915 (1987)

130. S. Grossman, U. Brosa, Z. Phys. A 319, 327 (1984)

131. S. Ayik, Z. Phys. A 292, 257 (1979)

132. P. Madler, Fiz. Elem. Chastits At. Yadra 15, 418 (1984)

133. P. Madler, Phys. Part. Nucl. 15, 190 (1984)

134. K.T.R. Davies, K.R.S. Devi, S.E. Koonin, M.R. Strayer, Treatise on Heavy-Ion Science, ed. by D.A. Bromley, vol. 3 (Plenum Press, New York, 1984), p. 2

135. V.M. Kolomiets, Local Density Approximation in Atomic and Nuclear Physics (Naukova Dumka, Kiev, 1990)

136. R.A. Broglia, A. Winther, Phys. Rep. 4, 153 (1972)

137. V.V. Sargsyan, Z. Kanokov, G.G. Adamian, N.V. Antonenko, Part. Nucl. 41, 175 (2010)

138. R.A. Kuzyakin, V.V. Sargsyan, G.G. Adamian, N.V. Antonenko, Part. Nucl. 48, 158 (2017)

139. Bing Wang, Kai Wen, Wei-Juan Zhao, En-Guang Zhao, Shan-Gui Zhou, At. Data Nucl. Data Tables 114, 281 (2017)

140. K. Dietrich, K. Hara, Nucl. Phys. A 211, 349 (1973)

141. G. Bertsch, R. Schäffer, Nucl. Phys. A 277, 509 (1977)

142. R.V. Jolos, R. Schmidt, J. Teichert, Nucl. Phys. A 249, 139 (1984)

143. D. Boose, J. Richert, Nucl. Phys. A 433, 511 (1985)

144. F. Catara, E.G. Lanza, Nucl. Phys. A 451, 299 (1986)

145. M. Baldo, J. Rapisarda, in The Response of Nuclei Under Extreme Conditions, ed. by R.A. Broglia (Pienum Press, New York, 1988), p. 201

146. S.P. Ivanova, R.V. Jolos, Nucl. Phys. A 530, 232 (1991)

147. R.V. Jolos, A.K. Nasirov, Yad. Fiz. 40, 721 (1984)

148. R.V. Jolos, A.K. Nasirov, Sov. J. Nucl. Phys. 40, 463 (1984)

149. R.V. Jolos, A.K. Nasirov, Yad. Fiz. 42, 175 (1985)

150. R.V. Jolos, A.K. Nasirov, Sov. J. Nucl. Phys. 42, 109 (1985)

151. R.V. Jolos, A.I. Muminov, A.K. Nasirov, Yad. Fiz. 44, 357 (1986)

152. R.V. Jolos, A.I. Muminov, A.K. Nasirov, Sov. J. Nucl. Phys. 44, $228(1986)$

153. N.V. Antonenko, R.V. Jolos, Yad. Fiz. 50, 98 (1989)

154. N.V. Antonenko, R.V. Jolos, Sov. J. Nucl. Phys. 50, 62 (1989)

155. N.V. Antonenko, R.V. Jolos, Yad. Fiz. 51, 690 (1989)

156. N.V. Antonenko, R.V. Jolos, Sov. J. Nucl. Phys. 51, 438 (1989)

157. G.G. Adamyan, R.V. Jolos, A.K. Nasirov, Yad. Fiz. 55, 660 (1992)

158. G.G. Adamyan, R.V. Jolos, A.K. Nasirov, Sov. J. Nucl. Phys. 55, $366(1992)$

159. G.G. Adamian, R.V. Jolos, A.K. Nasirov, Z. Phys. A 347, 203 (1994)

160. G.G. Adamian, N.V. Antonenko, R.V. Jolos, A.K. Nasirov, Nucl. Phys. A 551, 321 (1993)

161. S. Ayik, W. Nörenberg, Z. Phys. A 309, 121 (1982)

162. K. Niita, W. Nörenberg, S.J. Wang, Z. Phys. A 326, 69 (1987)

163. M. Tohyama, A.S. Umar, Phys. Rev. C 93, 034607 (2016)

164. C. Simenel, Phys. Rev. Lett. 106, 112502 (2011)

165. H.S. Köhler, Nucl. Phys. A 343, 315 (1980) 
166. H.S. Köhler, Nucl. Phys. A 378, 181 (1982)

167. G.G. Adamyan, N.V. Antonenko, R.V. Jolos, A.K. Nasirov, Phys. Part. Nucl. 25, 583 (1994)

168. T.C. Awes, R.L. Ferguson, R. Novotny, F.E. Obenshain, F. Plasil, S. Pontoppidan, V. Rauch, G.R. Young, H. Sann, Phys. Rev. Lett. 52, 251 (1984)

169. R. Vandenbosch, A. Lazzarini, D. Leach, D.-K. Lock, A. Ray, A. Seamster, Phys. Rev. Lett. 52, 1964 (1984)

170. J. Töke, W.U. Schröder, Ann. Rev. Nucl. Part. Sci. 42, 401 (1992)

171. H. Keller, R. Bellwied, K. Lützenkirchen, J.V. Kratz, W. Brüchle, H. Gäggler, K.J. Moody, M. Schädel, G. Wirth, Z. Phys. A 328, 255 (1987)

172. G. Beier, J. Friese, W. Henning, P. Kienle, H.J. Körner, W. Wagner, W.A. Mayer, W. Mayer, Z. Phys. A 336, 217 (1990)

173. K. Nashinohara, N. Takigawa, Z. Phys. A 324, 139 (1986)

174. A. Diaz-Torres, N.V. Antonenko, W. Scheid, Nucl. Phys. A 652, 61 (1999)

175. A. Diaz-Torres, G.G. Adamian, N.V. Antonenko, W. Scheid, Phys. Lett. B 481, 228 (2000)

176. C.-Y. Wong, Phys. Rev. Lett. 31, 766 (1973)

177. G.G. Adamian, N.V. Antonenko, R.V. Jolos, S.P. Ivanova, O.I. Melnikova, Int. J. Mod. Phys. E 5, 191 (1996)

178. A.S. Umar, V.E. Oberacker, Phys. Rev. C 74, 021601(R) (2006)

179. A.S. Umar, V.E. Oberacker, J.A. Maruhn, P.-G. Reinhard, Phys. Rev. C 81, 064607 (2010)

180. C. Simenel, R. Keser, A.S. Umar, V.E. Oberacker, Phys. Rev. C 88, 024617 (2013)

181. T.K. Steinbach, J. Vadas, J. Schmidt, C. Haycraft, S. Hudan, R.T. deSouza, L.T. Baby, S.A. Kuvin, I. Wiedenhöver, A.S. Umar, V.E. Oberacker, Phys. Rev. C 90, 041603(R) (2014)

182. A.S. Umar, V.E. Oberacker, J.A. Maruhn, P.-G. Reinhard, Phys. Rev. C 80, 041601(R) (2009)

183. K. Washiyama, D. Lacroix, Phys. Rev. C 78, 024610 (2008)

184. K. Washiyama, D. Lacroix, S. Ayik, Phys. Rev. C 79, 024609 (2009)

185. S. Ayik, Phys. Lett. B 658, 174 (2008)

186. S. Ayik, O. Yilmaz, B. Yilmaz, A.S. Umar, A. Gokalp, G. Turan, D. Lacroix, Phys. Rev. C 91, 054601 (2015)

187. B. Yilmaz, S. Ayik, D. Lacroix, K. Washiyama, Phys. Rev. C 83, 064615 (2011)

188. S. Ayik, K. Washiyama, D. Lacroix, Phys. Rev. C 79, 054606 (2009)

189. K. Washiyama, S. Ayik, D. Lacroix, Phys. Rev. C 80, 031602 (2009)

190. D.R. Benton, H. Breuer, F. Khazaie, K. Kwiatkowski, V.E. Viola, S. Bradley, A.C. Mignerey, A.P. Weston-Dawkes, Phys. Rev. C 38, 1207 (1988)

191. J. Tõke, W.U. Schröder, J.R. Huizenga, Phys. Rev. C 40, R1577 (1989)

192. D. Pade, W.U. Schröder, J. Tõke, J.L. Wile, R.T. DeSouza, Phys. Rev. C 43, 1288 (1991)

193. G.G. Adamian, R.V. Jolos, A.K. Nasirov, A.I. Muminov, Phys. Rev. C 53, 871 (1996)

194. N. Wang, Zh Li, X. Wu, J. Tian, Y. Zhang, M. Liu, Phys. Rev. C 69, 034608 (2004)

195. Kai Wen, F. Sakata, Zhu-Xia Li, Xi-Zhen Wu, Ying-Xun Zhang, Shan-Gui Zhou, Phys. Rev. Lett. 111, 012501 (2013)

196. Kai Wen, F. Sakata, Zhu-Xia Li, Xi-Zhen Wu, Ying-Xun Zhang, Shan-Gui Zhou, Phys. Rev. C 90, 054613 (2014)

197. E. Vigezzi, A. Winther, Ann. Phys. (NY) 192, 432 (1989)

198. A. Winther, Nucl. Phys. A 572, 191 (1994)

199. A. Winther, Nucl. Phys. A 594, 203 (1995)

200. A. Winther, program GRAZING. http://www.to.infn.it/ nanni/ grazing

201. Lu Gao-Feng Dai, En-Guang Zhao Guo, Shan-Gui Zhou, Phys. Rev. C 90, 044609 (2014)
202. A. Diaz-Torres, G.G. Adamian, N.V. Antonenko, W. Scheid, Phys. Rev. C 64, 024604 (2001)

203. V.I. Zagrebaev, A.V. Karpov, W. Greiner, Phys. Rev. C 85, 014608 (2012)

204. I. Stefan et al., Phys. Lett. B 779, 456 (2018)

205. N.V. Antonenko, R.V. Jolos, Z. Phys. A 338, 423 (1991)

206. G. Audi, O. Bersillan, J. Blachot, A.H. Wapstra, Nucl. Phys. A 729, 3 (2003)

207. P. Möller, J.R. Nix, W.D. Myers, W.J. Swiatecki, At. Data Nucl. Data Tables 59, 185 (1995)

208. S. Raman, C.W. Nester, P. Tikkanen, At. Data Nucl. Data Tables 78, 1 (2001)

209. L. Corradi, A.M. Vinodkumar, A.M. Stefanini, E. Fioretto, G. Prete, S. Beghini, G. Montagnoli, F. Scarlassara, G. Pollarolo, F. Cerutti, Aage Winther, Phys. Rev. C 66, 024606 (2002)

210. L. Corradi, G. Pollarolo, S. Szilner, J. Phys. G 36, 113101 (2009)

211. L. Corradi, A.M. Stefanini, C.J. Lin, S. Beghini, G. Montagnoli, F. Scarlassara, G. Pollarolo, A. Winther, Phys. Rev. C 59, 261 (1999)

212. S. Szilner et al., Phys. Rev. C 71, 044610 (2005)

213. ShA Kalandarov, G.G. Adamian, N.V. Antonenko, W. Scheid, Phys. Rev. C 82, 044603 (2010)

214. S.A. Kalandarov, G.G. Adamian, N.V. Antonenko, W. Scheid, J.P. Wieleczko, Phys. Rev. C 84, 064601 (2011)

215. A. Korgul et al., Phys. Rev. C 77, 034301 (2008)

216. ShA Kalandarov, G.G. Adamian, N.V. Antonenko, J.P. Wieleczko, Phys. Rev. C 90, 024609 (2014)

217. A.G. Artukh, V.V. Avdeichikov, G.F. Gridnev, V.L. Mikheev, V.V. Volkov, J. Wilczynski, Phys. Lett. B 31, 129 (1970)

218. A.G. Artukh, V.V. Avdeichikov, L.P. Chelnokov, G.F. Gridnev, V.L. Mikheev, V.I. Vakatov, V.V. Volkov, J. Wilczynski, Phys. Lett. B 32, 49 (1970)

219. P. Auger, T.H. Chiang, J. Galin, B. Gatty, D. Guerreau, E. Nolte, J. Pouthas, X. Tarrago, J. Girard, Z. Phys. A 289, 255 (1979)

220. D. Guerreau, J. Galin, B. Gatty, X. Tarrago, J. Girard, R. Lucas, C. Ngô, Z. Phys. A 295, 105 (1980)

221. H. Breuer et al., Phys. Rev. C 22, 2454 (1980)

222. R. Kirchner et al., Nucl. Phys. A 378, 549 (1982)

223. K. Rykaczewski, R. Kirchner, W. Kurcewicz, D. Schardt, N. Kaffrell, P. Peuser, E. Runte, W.-D. Schmidt-Ott, P. TidemandPetersson, K.-L. Gippert, Z. Phys. A 309, 273 (1983)

224. E. Runte et al., Nucl. Phys. A 399, 163 (1983)

225. U. Bosch et al., Phys. Lett. B 164, 22 (1985)

226. E. Runte, F. Meissner, V. Freystein, T. Hild, H. Salewski, W.-D. Schmidt-Ott, R. Michaelsen, Z. Phys. A 328, 373 (1987)

227. R.M. Chasteler, J.M. Nitschke, R.B. Firestone, K.S. Vierinen, P.A. Wilmarth, A.A. Shihab-Eldin, Z. Phys. A 332, 239 (1989)

228. K. Rykaczewski et al., Nucl. Phys. A 499, 529 (1989)

229. K. Becker et al., Nucl. Phys. A 522, 557 (1991)

230. C. Bruske, K.H. Burkard, W. Huller, R. Kirchner, O. Klepper, E. Roeckl, Nucl. Instrum. Methods Phys. Res. 186, 61 (1981)

231. Ch. Wennemann, W.-D. Schmidt-Ott, T. Hild, K. Krumbholz, V. Kunze, F. Meissner, H. Keller, R. Kirchner, E. Roeckl, Z. Phys. A 347, 185 (1994)

232. K.-L. Gippert et al., Nucl. Phys. A 453, 1 (1986)

233. M. Schädel et al., Phys. Rev. Lett. 41, 469 (1978)

234. M. Schädel et al., Phys. Rev. Lett. 48, 852 (1982)

235. M. Schädel et al., Phys. Rev. C 33, 1547 (1986)

236. H. Gäggeler et al., Phys. Rev. C 33, 1983 (1986)

237. D. Lee, H. von Gunten, B. Jacak, M. Nurmia, Yuan-fang Liu, Cheng Luo, G.T. Seaborg, D.C. Hoffman, Phys. Rev. C 25, 286 (1982)

238. D.C. Hoffman et al., Phys. Rev. C 31, 1763 (1985)

239. H.M. Devaraja et al., Eur. Phys. J. A 55, 25 (2019)

240. G.G. Adamian, N.V. Antonenko, V.V. Sargsyan, W. Scheid, A.S. Zubov, Phys. Rev. C 82, 017601 (2010) 
241. C. Simenel, Phys. Rev. Lett. 105, 192701 (2010)

242. H.M. Devaraja et al., Phys. Lett. B 748, 199 (2015)

243. S. Heinz et al., Eur. Phys. J. A 52, 278 (2016)

244. A. Di Nitto et al., Phys. Lett. B 784, 199 (2018)

245. C.-C. Sahm et al., Nucl. Phys. A 441, 316 (1985)

246. M. Veselsky et al., Proceedings of the Third International Conference on Dynamical Aspects of Nuclear Fission, CastaPapernicka, 1996, ed. by J. Kliman, B.I. Pustylnik (JINR, Dubna, 1997), p. 129

247. D. Velmeulen et al., Z. Phys. A 318, 157 (1984)

248. K. Nishio, H. Ikezoe, S. Mitsuoka, K. Satou, C.J. Lin, Phys. Rev. C 68, 064305 (2003)

249. K. Satou, H. Ikezoe, S. Mitsuoka, K. Nishio, C.J. Lin, S.C. Jeong, Phys. Rev. C 73, 034609 (2006)

250. K. Satou, H. Ikezoe, S. Mitsuoka, K. Nishio, S.C. Jeong, Phys. Rev. C 65, 054602 (2002)

251. A.N. Andreyev, D.D. Bogdanov, V.I. Chepigin, A.P. Kabachenko, S. Sharo, G.M. Ter-Akopian, A.V. Yeremin, O.N. Malyshev, Z. Phys. A 337, 231 (1990)

252. A.N. Andreyev, D.D. Bogdanov, V.I. Chepigin, A.P. Kabachenko, O.N. Malyshev, A.G. Popeko, R.N. Sagaidak, G.M. Ter-Akopian, M. Veselsky, A.V. Yeremin, Z. Phys. A 347, 225 (1994)

253. H.B. Yang et al., Eur. Phys. J. A 51, 88 (2015)

254. O.N. Malyshev et al., Eur. Phys. J. A 8, 295 (2000)

255. A.P. Leppänen et al., Phys. Rev. C 75, 054307 (2007)

256. A.N. Andreyev, D.D. Bogdanov, V.I. Chepigin, A.P. Kabachenko, O.N. Malyshev, G.M. Ter-Akopian, A.V. Yeremin, Z. Phys. A 338, 363 (1991)

257. F.P. Heßberger et al., Z. Phys. A 333, 111 (1989)

258. A.V. Yeremin et al., Nucl. Instrum. Methods A 350, 608 (1994)

259. Z. Kalaninova, S. Antalic, F.P. Heßberger, D. Ackermann, B. Andel, B. Kindler, M. Laatiaoui, B. Lommel, J. Maurer, Phys. Rev. C 92, 014321 (2015)

260. J.S. Barrett et al., Phys. Rev. C 91, 064615 (2015)

261. V.V. Desai et al., Phys. Rev. C 99, 044604 (2019)

262. W. Krolas, R. Broda, B. Fornal, T. Pawłat, H. Grawe, K.H. Maier, M. Schramm, R. Schubart, Nucl. Phys. A 724, 289 (2003)

263. W. Krolas et al., Nucl. Phys. A 832, 170 (2010)

264. O. Beliuskina et al., Eur. Phys. J. A 50, 161 (2014)

265. Y.X. Watanabe et al., Phys. Rev. Lett. 115, 172503 (2015)

266. E. Casarejos et al., Phys. Rev. C 74, 044612 (2006)

267. T. Kurtukian-Nieto, PhD thesis work (University of Santiage de Compostela, Spain, 2007)

268. M. Pfützner et al., Phys. Lett. B 444, 32 (1998)

269. H. Alvarez-Pol et al., Phys. Rev. C 82, 041602 (2010)

270. J. Kurcewicz et al., Phys. Lett. B 717, 371 (2012)

271. W.N. Catford, J. Phys. G. 24, 1377 (1998)

272. J. Simpson et al., Acta Phys. Hung. A Heavy Ion Phys. 11, 159 (2000)

273. W. Mayer, G. Beier, J. Friese, W. Henning, P. Kienle, H.J. Körner, W.A. Mayer, L. Müller, G. Rosner, W. Wagner, Phys. Lett. B 152, $162(1985)$

274. W. Wagner, W. Mayer, G. Beier, J. Friese, W. Henning, P. Kienle, H.J. Körner, Phys. Lett. B 196, 117 (1987)

275. T. Göbel, Study of nucleon transfer reactions for the synthesis of isotopes along the $\mathrm{N}=126$ shell. Bachelor Thesis, Justus-LiebigUniversity Giessen (2019)

276. E.M. Kozulin et al., Phys. Rev. C 86, 044611 (2012)

277. E.M. Kozulin et al., Phys. Rev. C 89, 014614 (2014)

278. E.M. Kozulin et al., Phys. Rev. C 96, 064621 (2017)

279. D. Guillemaud-Mueller et al., Z. Phys. A 332, 189 (1989)

280. D. Guillemaud-Mueller et al., Phys. Rev. C 41, 937 (1990)

281. M. Lewitowicz et al., Phys. Lett. B 332, 20 (1994)

282. H. Sakurai et al., Phys. Rev. C 54, R2802 (1996)

283. R. Scheider et al., Z. Phys. A 348, 241 (1994)

284. M. Fauerbach et al., Phys. Rev. C 53, 647 (1996)
285. O.B. Tarasov et al., Phys. Lett. B 409, 64 (1997)

286. H. Sakurai et al., Phys. Lett. B 448, 180 (1999)

287. S.M. Lukyanov et al., J. Phys. G 28, L41 (2002)

288. M. Notani et al., Phys. Lett. B 542, 49 (2002)

289. O.B. Tarasov et al., Phys. Rev. C 75, 064613 (2007)

290. T. Baumann et al., Nature 449, 1022 (2007)

291. M. Mocko et al., (2008). arXiv:0804.2603v2 [nucl-ex]

292. B.M. Quednau et al., Phys. Lett. B 309, 10 (1993)

293. V. Borrel et al., Z. Phys. A 314, 191 (1983)

294. J.F. LeColley et al., Phys. Lett. B 325, 317 (1994)

295. B.J. Charity et al., Z. Phys. A 341, 53 (1991)

296. D. Guerreau et al., Phys. Lett. B 131, 293 (1983)

297. F. Rami et al., Z. Phys. A 318, 239 (1984)

298. F. Rami et al., Nucl. Phys. A 444, 325 (1985)

299. M.C. Mermaz et al., Phys. Rev. C 31, 1972 (1985)

300. M.C. Mermaz et al., Nucl. Phys. A 441, 129 (1985)

301. V. Borrel et al., Z. Phys. A 324, 205 (1986)

302. F. Pougheon et al., Z. Phys. A 327, 17 (1987)

303. B. Borderie et al., Phys. Lett. B 205, 26 (1988)

304. L. Stuttge et al., Nucl. Phys. A 539, 511 (1992)

305. A. Sokolov et al., Nucl. Phys. A 562, 273 (1993)

306. G.A. Souliotis, D.J. Morrissey, N.A. Orr, B.M. Sherrill, J.A. Winger, Phys. Rev. C 46, 1383 (1992)

307. R. Pfaff et al., Phys. Rev. C 51, 1348 (1995)

308. M. Mocko et al., Phys. Rev. C 74, 054612 (2006)

309. K. Summerer et al., Phys. Rev. C 42, 2546 (1990)

310. G.G. Adamian, N.V. Antonenko, W. Scheid, Nucl. Phys. A 618, $176(1997)$

311. M.B. Tsang et al., Phys. Rev. C 76, 041302(R) (2007)

312. M. Wiescher, F. Käppeler, K. Langanke, Ann. Rev. Astron. Astrophys. 50, 165 (2012)

313. B.B. Back, H. Esbensen, C.L. Jiang, K.E. Rehm, Rev. Mod. Phys. 86, 317 (2014)

314. L.F. Canto, P.R.S. Gomes, R. Donangelo, M.S. Hussein, Phys. Rep. 596, 1 (2015)

315. M. Boselli, A. Diaz-Torres, J. Phys. G 41, 094001 (2014)

316. M.A. Reimann, P.W. Martin, E.W. Vogt, Can. J. Phys. 46, 2241 (1968)

317. A. Diaz-Torres, I.J. Thompson, W. Scheid, Phys. Lett. B 533, 265 (2002)

318. A. Diaz-Torres, I.J. Thompson, W. Scheid, Nucl. Phys. A 703, 83 (2002)

319. A. Shrivastava, A. Navin, N. Keeley, K. Mahata, K. Ramachandran, V. Nanal, V.V. Parkar, A. Chatterjee, S. Kailasa, Phys. Lett. B 633, 463 (2006)

320. R. Rafiei, R. du Rietz, D.H. Luong, D.J. Hinde, M. Dasgupta, M. Evers, A. Diaz-Torres, Phys. Rev. C 81, 024601 (2010)

321. D.H. Luong, M. Dasgupta, D.J. Hinde, R. du Rietz, R. Rafiei, C.J. Lin, M. Evers, A. Diaz-Torres, Phys. Lett. B 695, 105 (2011)

322. D.H. Luong, M. Dasgupta, D.J. Hinde, R. du Rietz, R. Rafiei, C.J. Lin, M. Evers, A. Diaz-Torres, Phys. Rev. C 88, 034609 (2013)

323. S. Kalkal et al., Phys. Rev. C 93, 044605 (2016)

324. K.J. Cook, E.C. Simpson, D.H. Luong, Sunil Kalkal, M. Dasgupta, D.J. Hinde, Phys. Rev. C 93, 064604 (2016)

325. P.P. Singh et al., Phys. Rev. C 80, 064603 (2009)

326. B.V. Carlson, T. Frederico, M.S. Hussein, Phys. Lett. B 767, 53 (2017)

327. J. Lei, A.M. Moro, Phys. Rev. Lett. 122, 042503 (2019)

328. K.J. Cook et al., Phys. Rev. Lett. 122, 102501 (2019)

329. K. Yabana, Prog. Theor. Phys. 97, 437 (1997)

330. M. Boselli, A. Diaz-Torres, Phys. Rev. C 92, 044610 (2015)

331. A. Diaz-Torres, I.J. Thompson, Phys. Rev. C 65, 024606 (2002)

332. A. Diaz-Torres, I.J. Thompson, C. Beck, Phys. Rev. C 68, 044607 (2003)

333. I.J. Thompson, A. Diaz-Torres, Prog. Theor. Phys. Suppl. 154, 69 (2004) 
334. A. Diaz-Torres, D.J. Hinde, J.A. Tostevin, M. Dasgupta, L.R. Gasques, Phys. Rev. Lett. 98, 152701 (2007)

335. A. Diaz-Torres, J. Phys. G 37, 075109 (2010)

336. A. Diaz-Torres, Comput. Phys. Commun. 182, 1100 (2011)

337. D.J. Hinde, M. Dasgupta, B.R. Fulton, C.R. Morton, R.J. Wooliscroft, A.C. Berriman, K. Hagino, Phys. Rev. Lett. 89, 272701 (2002)

338. A. Shrivastava et al., Phys. Lett. B 718, 931 (2013)

339. Rafael Van den Bossche, Alexis Diaz-Torres, Phys. Rev. C 100, 044604 (2019)

340. R. Kharab, R. Chahal, R. Kumar, Nucl. Phys. A 960, 11 (2017)

341. A. Diaz-Torres, D. Quraishi, Phys. Rev. C 97, 024611 (2018)

342. M. Dasgupta, P.R.S. Gomes, D.J. Hinde, S.B. Moraes, R.M. Anjos, A.C. Berriman, R.D. Butt, N. Carlin, J. Lubian, C.R. Morton, J.O. Newton, A. Szanto de Toledo, Phys. Rev. C 70, 024606 (2004)

343. G.G. Adamian, N.V. Antonenko, D. Lacroix, Phys. Rev. C 82, 064611 (2010)

344. O. Kofoed-Hansen, Proc. 3rd Int. Conf. on Nuclei Far from Stability, Cargèse, France, 1976. CERN Report 76-13 (1976)

345. T.J.M. Symons, Y.P. Viyogi, G.D. Westfall, P. Doll, D.E. Greiner, H. Faraggi, P.J. Lindstrom, D.K. Scott, H.J. Crawford, C. McParland, Phys. Rev. Lett. 42, 40 (1979)

346. ISOLDE nuclide chart, http://isoyields-classic.web.cern.ch

347. M.V. Ricciardi et al., Eur. Phys. J. Spec. Top. 150(1), 321 (2007)

348. D. Habs et al., Radioactive beam experiments at ISOLDE: Coulomb excitation and neutron transfer reactions of exotic nuclei. Proposal to the ISOLDE committee, CERN/ISC 94-25 ISC/P68 (1994)

349. M. Lindroos, P.A. Butler, M. Huyse, K. Riisager, Nucl. Instrum. Methods B 266, 4687 (2008)

350. M.J.G. Borge, K. Riisager, Eur. J. Phys. A 52, 334 (2016)

351. H. Geissel et al., Nucl. Instrum. Methods B 204, 71 (2003)

352. M. Winkler et al., Nucl. Instrum. Methods B 266, 4183 (2008)

353. J.S. Winfield, private communications

354. A. Gade, B.M. Sherrill, Phys. Scr. 91, 053003 (2016)

355. S. Schwarz et al., Nucl. Instrum. Methods B 317, 463 (2013)

356. B. Franzke, Nucl. Instrum. Methods B 24/25, 18 (1987)

357. J.W. Xia et al., Nucl. Instrum. Methods A 488, 11 (2002)

358. R. Smolanczuk, A. Sobiczewski, Proceedings of the XV Nuclear Physics Divisional Conference on Low Energy Nuclear Dynamics, ed. by Yu.T. Oganessian et al. (World Scientific, Singapore, 1995), p. 313
359. M. Bender, K. Rutz, P.-G. Reinhard, J.A. Maruhn, W. Greiner, Phys. Rev. C 60, 034304 (1999)

360. M. Bender, Phys. Rev. C 61, 031302 (2000)

361. S. Cwiok, W. Nazarewicz, P.H. Heenen, Phys. Rev. Lett. 83, 1108 (1999)

362. S. Cwiok, P.-H. Heenen, W. Nazarewicz, Nature 433, 705 (2005)

363. G.G. Adamian, N.V. Antonenko, W. Scheid, Phys. Rev. C 69, 044601 (2004)

364. YuTs Oganessian et al., Phys. Rev. Lett 104, 142502 (2010)

365. YuTs Oganessian et al., Phys. Rev. C 76, 011601 (2007)

366. YuTs Oganessian et al., Phys. Rev. C 69, 054607 (2004)

367. W.D. Loveland, Phys. Rev. C 76, 014612 (2007)

368. W.D. Loveland, Front. Phys. 7, 1 (2019)

369. S. Heinz et al., Study of the Dinuclear System ${ }^{A} R b+{ }^{209} B i$ $\left(Z_{1}+Z_{2}=120\right)$, Proposal to the ISOLDE committee, CERNINTC-2012-043, INTC-P-344 (2012)

370. K. Knie, Secondary beams and targets, The CERN Accelerator School CAS, 16-29 September 2018, Constanta

371. Y. Kadi et al., J. Phys. G 44, 084003 (2017)

372. H. Geissel et al., Nucl. Instrum. Methods B 70, 286 (1992)

373. J. Äystö et al., Nucl. Instrum. Methods B 376, 111 (2016)

374. J.Y. Yano, Nucl. Instrum. Methods B 261, 1009 (2007)

375. S. Gales, Nucl. Phys. A 834, 717c (2010)

376. W.R. Plaß et al., Nucl. Instrum. Methods B 317, 457 (2013)

377. T. Dickel et al., Nucl. Instrum. Methods A 777, 172 (2015)

378. T. Dickel et al., Reaction studies with the FRS Ion Catcher: A novel approach and universal method for the production, identification of and experiments with unstable isotopes produced in multinucleon transfer reactions, Proposal to the G-PAC 2018/2019, proposal number $\mathrm{S} 475$

379. S. Kimura, H. Ishiyama, H. Miyatake, Y. Hirayama, Y.X. Watanabe, H.S. Jung, M. Oyaizu, M. Mukai, S.C. Jeong, A. Ozawa, Nucl. Instrum. Methods B 376, 338 (2015)

380. Y. Hirayama et al., EPJ Web. Conf. 109, 08001 (2016)

381. J.M. Gates et al., Phys. Rev. Lett. 121, 222501 (2018)

382. Juhee Hong, G.G. Adamian, N.V. Antonenko, Phys. Rev. C 94, 044606 (2016)

383. Juhee Hong, G.G. Adamian, N.V. Antonenko, Phys. Rev. C 96, 014609 (2017)

384. K. Siwek-Wilczynska, T. Cap, M. Kowal, Phys. Rev. C 99, 054603 (2019)

385. A. Lopez-Martens et al., Phys. Lett. B 795, 271 (2019) 